The Journal of Fourier Analysis and Applications

Volume 13, Issue 2, 2007

\title{
History and Evolution of the Density Theorem for Gabor Frames
}

\author{
Christopher Heil \\ Communicated by Hans G. Feichtinger \\ Research Survey
}

\begin{abstract}
The Density Theorem for Gabor Frames is one of the fundamental results of time-frequency analysis. This expository survey attempts to reconstruct the long and very involved history of this theorem and to present its context and evolution, from the onedimensional rectangular lattice setting, to arbitrary lattices in higher dimensions, to irregular Gabor frames, and most recently beyond the setting of Gabor frames to abstract localized frames. Related fundamental principles in Gabor analysis are also surveyed, including the Wexler-Raz biorthogonality relations, the Duality Principle, the Balian-Low Theorem, the Walnut and Janssen representations, and the Homogeneous Approximation Property. An extended bibliography is included.
\end{abstract}

\section{Introduction}

\subsection{Background and Motivation}

Frames provide robust, basis-like (but generally nonunique) representations of vectors in a Hilbert space $H$. The potential redundancy of frames often allows them to be more easily constructible than bases, and to possess better properties than are achievable using bases. For example, redundant frames offer more resilience to the effects of noise or to erasures of frame elements than do bases. Frames were introduced by Duffin and Schaeffer [57] in the context of nonharmonic Fourier series, and today they have applications in a wide

Math Subject Classifications. 42C15, 42C30, 46C99.

Keywords and Phrases. Balian-Low Theorem, complete sequence, density theorem, duality principle, frames, Gabor system, homogeneous approximation property, Janssen representation, localized frames, modulation spaces, Riesz bases, Schauder bases, time-frequency analysis, von Neumann algebras, Walnut representation, Wexler-Raz biorthogonality relations, Weyl-Heisenberg system Acknowledgments and Notes. The author was partially supported by NSF Grant DMS-0139261. 
range of areas, including, for example, sampling theory [1], operator theory [100], nonlinear sparse approximation [84], pseudodifferential operators [94], wavelet theory [49], wireless communications [171], data transmission with erasures [83], signal processing [24], and quantum computing [58]. A frame which provides unique representations is called a Riesz basis.

Gabor frames are a particular type of frame whose elements are simply generated by time-frequency shifts of a single window function or atom. Specifically, if $g \in L^{2}\left(\mathbf{R}^{d}\right)$ is a fixed function and $\Lambda$ is a sequence of points in $\mathbf{R}^{2 d}$, then the Gabor system or WeylHeisenberg system generated by $g$ and $\Lambda$ is the set of time-frequency shifts of $g$ along $\Lambda$ given by

$$
\mathcal{G}(g, \Lambda)=\left\{M_{\xi} T_{x} g\right\}_{(x, \xi) \in \Lambda}=\left\{e^{2 \pi i \xi \cdot t} g(t-x)\right\}_{(x, \xi) \in \Lambda} .
$$

Here $T_{x}$ is the translation operator $T_{x} g(t)=g(t-x), M_{\xi}$ is the modulation operator $M_{\xi} g(t)=e^{2 \pi i \xi \cdot t} g(t)$, and the compositions $T_{x} M_{\xi}$ or $M_{\xi} T_{x}$ are time-frequency shift operators (illustrated in Figure 1). Often, the index set $\Lambda$ is required to possess extra structure, e.g., it may be a lattice $A\left(\mathbf{Z}^{2 d}\right)$ where $A$ is an invertible matrix, or a rectangular lattice $\alpha \mathbf{Z}^{d} \times \beta \mathbf{Z}^{d}$. If the Gabor system is a frame then we call it a Gabor frame.

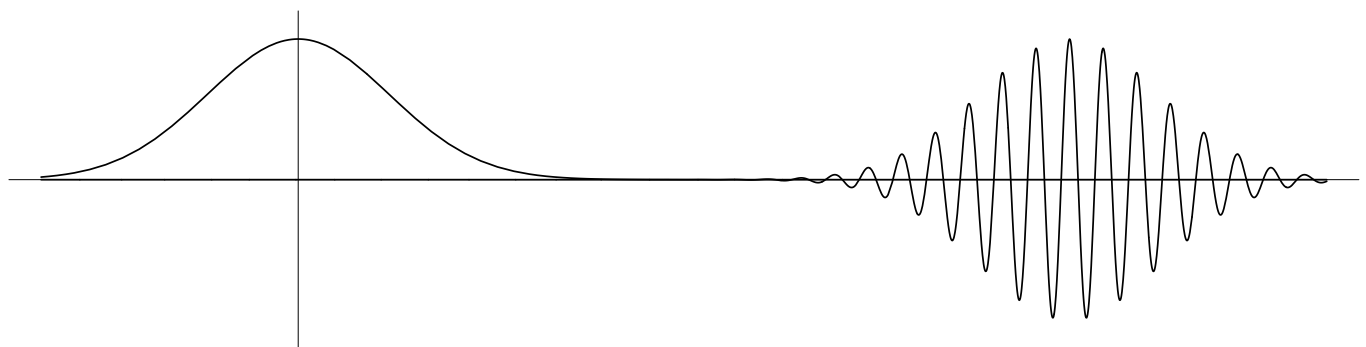

FIGURE 1 A window $g$ and the real part of a time-frequency shift $M_{\xi} T_{x} g$ of $g$.

The structure of Gabor frames makes them especially suitable for applications involving time-dependent frequency content. Music is one natural example [56], but in fact time-frequency is ubiquitous in mathematics, science, and engineering. For example, due to multipath propagation and Doppler shifts, wireless communications channels can be modeled as superpositions of time-frequency shift operators, which in mathematical terms are pseudodifferential operators. Gabor frames are a natural tool for analyzing pseudodifferential operators; for example see [94, 104] for applications to the theory of pseudodifferential operators, [170] for applications to mobile communications, [135] for the use of Gabor frames in pseudodifferential operators in engineering (where they are called time-varying filters), and [89, Chapter 14] for a wealth of discussion on pseudodifferential operators in mathematics, engineering, and physics (where they are called quantization rules).

As can be expected from its multitudes of uses, Gabor theory has a long history. Gröchenig [89, Section 7.5] and Janssen [127] point out that von Neumann [153, p. 406] claimed (without proof) that, for the Gaussian window $\varphi(t)=2^{1 / 4} e^{-\pi t^{2}}$ and the lattice $\Lambda=\mathbf{Z}^{2}$ (here $d=1$ ), the Gabor system $\mathcal{G}\left(\varphi, \mathbf{Z}^{2}\right)$ is complete in $L^{2}(\mathbf{R})$, i.e., its finite linear span is dense. Gabor conjectured that every function in $L^{2}(\mathbf{R})$ can actually be represented in the form

$$
f=\sum_{k, n \in \mathbf{Z}} c_{k n}(f) M_{n} T_{k} \varphi
$$


for some scalars $c_{k n}(f)$; see [80, Eq. 1.29]. This is one reason why general families $\mathcal{G}(g, \Lambda)$ are named in honor of Gabor.

Von Neumann's claim of completeness was proved in [15, 157], and [5]. However, completeness of $\mathcal{G}\left(\varphi, \mathbf{Z}^{2}\right)$ is only a weak property: It implies that each $f$ can be approximated arbitrarily well in $L^{2}$-norm by a finite linear combination of time-frequency shifts of $\varphi$, but the coefficients needed in these linear combinations to achieve better and better approximations can be entirely unrelated to each other. Completeness does not imply expansions of the form given in (1.2).

Reading perhaps a bit extra into what von Neumann and Gabor actually wrote, possibly they expected that the expansions (1.2) would exist with convergence in the norm of $L^{2}$ and with scalars uniquely determined by $f$. In modern terminology, this would say that $\mathcal{G}\left(\varphi, \mathbf{Z}^{2}\right)$ forms a Schauder basis for $L^{2}(\mathbf{R})$. However, even a Schauder basis is not sufficient in practice - we also need to know that the expansions in (1.2) are "stable" and "robust." At the very least we need unconditional convergence of the basis expansions (i.e., independent of ordering), and we need the coefficients $c_{k n}(f)$ to stably encode the norm of $f$, meaning that their $\ell^{2}$-norm should form an equivalent norm for $L^{2}(\mathbf{R})$. In modern terminology, this would be the case if $\mathcal{G}\left(\varphi, \mathbf{Z}^{2}\right)$ forms a Riesz basis for $L^{2}(\mathbf{R})$.

Neither of these can be literally true, for the Gabor system $\mathcal{G}\left(\varphi, \mathbf{Z}^{2}\right)$ is overcompleteany single element may be removed and the resulting system will still be complete (though it becomes incomplete if two elements are removed). But even with one element removed, the system forms neither a Schauder basis nor a Riesz basis, cf. [73, p. 168]. In fact, Janssen proved in [118] that Gabor's conjecture that each $f \in L^{2}\left(\mathbf{R}^{d}\right)$ has an expansion of the form in (1.2) is true, but he also showed that the series converges only in the sense of tempered distributions - not in the norm of $L^{2}$-and that the coefficients $c_{k n}$ may grow with $k$ and $n$ (see also [151]).

Today we realize that there simply are no "good" Gabor Riesz bases $\mathcal{G}\left(g, \alpha \mathbf{Z}^{d} \times \beta \mathbf{Z}^{d}\right)$ for $L^{2}\left(\mathbf{R}^{d}\right)$. Indeed, the Balian-Low Theorem, which we will discuss in Section 3.7 below, implies that if $\mathcal{G}\left(g, \alpha \mathbf{Z}^{d} \times \beta \mathbf{Z}^{d}\right)$ is a Riesz basis then the window $g$ must be very poorly concentrated in the time-frequency plane. In particular, without good time-frequency concentration, such a basis cannot be used to analyze properties of functions other than the $L^{2}$-norm.

On the other hand, for some (but not all) applications we may be able to forego the luxury of unique expansions. Daubechies, Grossmann, and Morlet revitalized interest in frame theory when they proved in [51] that for each $\alpha, \beta>0$ which satisfy $\alpha \beta<1$ there do exist some functions $g$ which are very well-localized in the time-frequency plane (e.g., $\left.g \in C_{c}^{\infty}(\mathbf{R})\right)$ such that $\mathcal{G}(g, \alpha \mathbf{Z} \times \beta \mathbf{Z})$ will form a frame but not a Riesz basis for $L^{2}(\mathbf{R})$. Consequently, for such windows we have for each $f \in L^{2}(\mathbf{R})$ that there exist coefficients $c_{k n}(f)$ such that

$$
f=\sum_{k, n \in \mathbf{Z}} c_{k n}(f) M_{\beta n} T_{\alpha k} g
$$

with unconditional convergence of the series in $L^{2}$-norm, with a prescribed formula for these coefficients $c_{k n}(f)$, and with the $\ell^{2}$-norm of the coefficients $c_{k n}(f)$ forming an equivalent norm for $L^{2}(\mathbf{R})$. The disadvantage is that the coefficients $c_{k n}(f)$ need not be unique, but often this is not an issue. Furthermore, Feichtinger and Gröchenig proved that a Gabor frame whose window is sufficiently well-localized in the time-frequency plane actually provides frame expansions not merely for $L^{2}\left(\mathbf{R}^{d}\right)$ but for an entire associated family of Banach function spaces whose norms quantify time-frequency concentration. These are the modulation spaces that are described in Section 2.3 below. 
Thus, redundant Gabor frames provide us with useful tools for many applications. Still, many questions are left unanswered. When will we have a Gabor frame - how can we recognize which $g$ and which index sets $\Lambda$ will actually yield Gabor systems that are frames? Can we determine necessary or sufficient conditions for a Gabor system to be complete, a frame, a Riesz basis, or a Riesz sequence (a Riesz basis for its closed span within $\left.L^{2}\left(\mathbf{R}^{d}\right)\right)$ ? Can these conditions be formulated to depend only on $g$ or only on $\Lambda$ ? If the window of a Gabor frame is well-localized in the time-frequency plane, will the dual frame also have an appropriate localization? Under what conditions will the Gabor frame expansions extend beyond the Hilbert space setting to the associated modulation spaces? These and other types of questions will be considered in this expository survey article.

\subsection{The Density Theorem}

The Density Theorem for Gabor Frames is one of the fundamental results in time-frequency analysis. It is not just one theorem, as it has evolved over time, from a result for the rigid setting of rectangular lattice Gabor systems in one dimension, to more general lattice systems in higher dimensions, then to arbitrary irregular Gabor systems, and most recently beyond the Gabor setting to abstract localized frames. The purpose of this survey is to discuss the history, context, and evolution of the Density Theorem. This history is long and intricate, and we attempt to present it as best that we have been able to reconstruct it, and to include all relevant original references that we are aware of. No new results appear here; rather our goal is to bring together in one place a comprehensive survey of results on Gabor theory related to or inspired by the theme of the Density Theorem (with a natural, and we hope forgivable, bias towards topics which the author has been most directly involved in). Daubechies article [48] and book [49], and most especially Gröchenig's text [89] have been invaluable resources in composing this article, but we have attempted wherever possible to verify all statements with the original sources. We also give references to survey articles on various topics where the reader can obtain further information; in particular, Janssen's survey [127] contains many references related to the early development of Gabor theory. All inaccuracies or omissions in this article are solely the fault of the author.

The Density Theorem essentially states that necessary conditions for a Gabor system $\mathcal{G}(g, \Lambda)$ to be complete, a frame, a Riesz basis, or a Riesz sequence can be formulated in terms of the index set $\Lambda$ alone-independently of the window $g$. In particular, $\Lambda$ cannot be spread "too sparsely" throughout the time-frequency plane $\mathbf{R}^{2 d}$, yet conversely cannot be "locally too dense" (in the sense that there must exist a finite maximum number of points of $\Lambda$ in any unit cube). Sparseness is quantified for a lattice system by the volume of the lattice, while in the irregular setting the notions of Beurling density provide a means of quantifying the average density of $\Lambda$. These concepts are defined precisely in the body of the article.

In addition to (and mostly prior to) the Density Theorem for Gabor frames, there is also a rich literature on closely related ideas and results in the settings of sampling and interpolation of band-limited functions, density conditions for systems of windowed exponentials, and sampling in the Bargmann-Fock space of entire functions. Indeed, the precise formulation of the Nyquist density is due to H. Landau $[143,144]$, in the context of sampling and interpolation of band-limited functions. We will not attempt to relate this additional history and context here, but refer to Seip's text [167] for additional information. 


\subsection{Outline}

This article is organized as follows. Section 2 presents some background and basic facts on frames and Gabor systems. We include background on the modulation and amalgam spaces, although these spaces enter the discussion only at certain points. In Section 3, we discuss the Density Theorem for the case of rectangular lattice Gabor systems in one dimension. Historically, this is the setting of the first results on the Density Theorem. In Section 4 we discuss the Density Theorem for arbitrary lattices in higher dimensions, including the special case of symplectic lattices. Section 5 is devoted to irregular Gabor systems, and Section 6 discusses the implications for Gabor systems of the recent results on the Density Theorem for the setting of abstract localized frames. Finally, we make a few remarks in Section 7 concerning density results for wavelet systems.

\section{Notation and Basic Facts}

For background on Gabor systems, frames, Riesz bases, and the modulation and amalgam spaces we recommend Gröchenig's text [89] and also [41, 189, 49, 113]. A brief review of basic facts and terminology is provided below.

Throughout, we use a Fourier transform normalized as $\hat{f}(\xi)=\int f(x) e^{-2 \pi i \xi \cdot x} d x$.

\subsection{Frames and Riesz Bases}

Definition/Facts 1. Let $\mathcal{F}=\left\{f_{i}\right\}_{i \in \mathbf{N}}$ be a sequence in a Hilbert space $H$.

(a) $\mathcal{F}$ is complete (or total or fundamental) if its finite linear span is dense in $H$. That is, for each $f \in H$ and each $N \in \mathbf{N}$ there exist scalars $\left\{c_{N, i}(f)\right\}_{i \in \mathbf{N}}$ such that $\sum_{i=1}^{N} c_{N, i}(f) f_{i} \rightarrow f$ as $N \rightarrow \infty$.

(b) $\mathcal{F}$ is minimal if there exists a sequence $\left\{\tilde{f}_{i}\right\}_{i \in \mathbf{N}}$ in $H$ that is biorthogonal to $\mathcal{F}$, i.e., $\left\langle f_{i}, \tilde{f}_{j}\right\rangle=\delta_{i j}$ for $i, j \in \mathbf{N}$. Equivalently, $\left\{f_{i}\right\}_{i \in \mathbf{N}}$ is minimal if $f_{j} \notin \overline{\operatorname{span}}\left\{f_{i}\right\}_{i \neq j}$ for each $j \in \mathbf{N}$. A sequence that is both minimal and complete is called exact. In this case the biorthogonal sequence is unique.

(c) $\left\{f_{i}\right\}_{i \in \mathbf{N}}$ is a Schauder basis if for each $f \in H$ there exist unique scalars $c_{i}(f)$ such that $f=\sum_{i=1}^{\infty} c_{i}(f) f_{i}$. All Schauder bases are exact, but not all exact sequences are Schauder bases.

(d) $\mathcal{F}$ is a Bessel sequence in $H$ if there exists a constant $B>0$, called a Bessel bound, such that

$$
\forall f \in H, \quad \sum_{i=1}^{\infty}\left|\left\langle f, f_{i}\right\rangle\right|^{2} \leq B\|f\|^{2} .
$$

In this case, the analysis map or coefficient map $C f=\left\{\left\langle f, f_{i}\right\rangle\right\}_{i \in \mathbf{N}}$ is a bounded map of $H$ into $\ell^{2}$, with $\|C\|^{2} \leq B$. Its adjoint is the synthesis map $C^{*}: \ell^{2} \rightarrow H$, and is given by $C^{*}\left(\left\{c_{i}\right\}_{i \in \mathbf{N}}\right)=\sum c_{i} f_{i}$ (this series converges unconditionally in the norm of $H$ ).

(e) $\mathcal{F}$ is a frame for $H$ if there exist constants $A, B>0$, called frame bounds, such that

$$
\forall f \in H, \quad A\|f\|^{2} \leq \sum_{i=1}^{\infty}\left|\left\langle f, f_{i}\right\rangle\right|^{2} \leq B\|f\|^{2} .
$$


The frame operator $S f=C^{*} C f=\sum_{i=1}^{\infty}\left\langle f, f_{i}\right\rangle f_{i}$ is a bounded, positive, and invertible mapping of $H$ onto itself. The canonical dual frame is $\tilde{\mathcal{F}}=\left\{\tilde{f}_{i}\right\}_{i \in \mathbf{N}}=\left\{S^{-1}\left(f_{i}\right)\right\}_{i \in \mathbf{N}}$. For each $f \in H$ we have the frame expansions

$$
f=\sum_{i=1}^{\infty}\left\langle f, f_{i}\right\rangle \tilde{f}_{i}=\sum_{i=1}^{\infty}\left\langle f, \tilde{f}_{i}\right\rangle f_{i}
$$

and these series converge unconditionally in the norm of $H$. Thus all frames are complete, but not all complete sequences are frames. All frames are norm-bounded above, with $\left\|f_{i}\right\|^{2} \leq B$ for all $i$.

(f) We say that a frame $\mathcal{F}$ is tight if we can take $A=B$ (in this case $S=A I$ ). A frame is Parseval if we can take $A=B=1$ (in this case $S=I$ ). A Parseval frame is self-dual, i.e., $\tilde{f}_{i}=f_{i}$.

(g) If $\mathcal{F}$ is a frame, then $\mathcal{F}^{\sharp}=\left\{S^{-1 / 2}\left(f_{i}\right)\right\}_{i \in \mathbf{N}}$ is a Parseval frame, called the canonical Parseval frame associated with $\mathcal{F}$.

(h) If $\mathcal{F}$ is a frame, then the following statements are equivalent:

(i) $\mathcal{F}$ is the image of an orthonormal basis under a continuous, invertible linear map of $H$ onto itself,

(ii) $\mathcal{F}$ is a Schauder basis,

(iii) the coefficients in (2.3) are unique for each $f \in H$,

(iv) $\mathcal{F}$ and its canonical dual frame $\tilde{\mathcal{F}}$ are biorthogonal.

In this case, $\mathcal{F}$ is called a Riesz basis.

(i) If $\mathcal{F}$ is a frame that is not a Riesz basis, then there exists a nonempty subset $J \subset \mathbf{N}$ such that $\mathcal{F}=\left\{f_{i}\right\}_{i \in \mathbf{N} \backslash J}$ is a frame for $H$ [57]. The excess of the frame is

$$
e(\mathcal{F})=\sup \left\{|J|:\left\{f_{i}\right\}_{i \in \mathbf{N} \backslash J} \text { is complete }\right\} .
$$

A frame has finite excess if and only if it is the union of a Riesz basis and a finite set [116]. If $e(\mathcal{F})=\infty$, there need not exist an infinite $J_{1} \subset I$ such that $\left\{f_{i}\right\}_{i \in \mathbf{N} \backslash J_{1}}$ is a frame. The frames for which this is true were characterized in [10]. In particular, the following statements are equivalent (note that $0 \leq\left\langle f_{i}, \tilde{f}_{i}\right\rangle \leq 1$ for all $i$ since $\left\langle f_{i}, \tilde{f}_{i}\right\rangle=\left\|S^{-1 / 2}\left(f_{i}\right)\right\|^{2}$ and $\left\{S^{-1 / 2}\left(f_{i}\right)\right\}_{i \in \mathbf{N}}$ is a Parseval frame).

(i) There exists an infinite $J_{1} \subset I$ such that $\left\{f_{i}\right\}_{i \in \mathbf{N} \backslash J_{1}}$ is a frame for $H$.

(ii) There exists an infinite subset $J_{2} \subset I$ and a constant $L>0$ such that for each $n \in J_{2}$ we have that $\left\{f_{i}\right\}_{i \neq n}$ is a frame for $H$ with lower frame bound $L$.

(iii) There exists an infinite subset $J_{3} \subset I$ such that $\sup _{i \in J_{3}}\left\langle f_{i}, \tilde{f}_{i}\right\rangle<1$.

(j) A sequence $\mathcal{F}=\left\{f_{i}\right\}_{i \in I}$ that is a frame for its closed linear span in $H$ is called a frame sequence. In this case the canonical dual frame sequence is its canonical dual frame within the closed subspace $\overline{\operatorname{span}}(\mathcal{F})$. Likewise, a Riesz sequence is a sequence that forms a Riesz basis for its closed linear span in $H$.

\subsection{Gabor Systems}

We will use the following terminology.

Definition 1. Let $g \in L^{2}(\mathbf{R})$ be given, and let $\Lambda$ be a sequence of points in $\mathbf{R}^{2 d}$. Let $\mathcal{G}(g, \Lambda)$ be the Gabor system defined in (1.1). 
(a) If $\Lambda=A\left(\mathbf{Z}^{2 d}\right)$ where $A$ is an invertible $2 d \times 2 d$ matrix, then we say that $\mathcal{G}\left(g, A\left(\mathbf{Z}^{2 d}\right)\right)$ is a lattice Gabor system. (Thus, for us a lattice will always mean a full-rank lattice.)

(b) If $\Lambda=\alpha \mathbf{Z}^{d} \times \beta \mathbf{Z}^{d}$ where $\alpha, \beta>0$, then we say that $\mathcal{G}\left(g, \alpha \mathbf{Z}^{d} \times \beta \mathbf{Z}^{d}\right)$ is a rectangular lattice Gabor system.

(c) If $\Lambda$ is not a lattice, then $\mathcal{G}(g, \Lambda)$ is an irregular Gabor system.

(d) If $\mathcal{G}(g, \Lambda)$ is a frame for $L^{2}\left(\mathbf{R}^{d}\right)$, then we call it a Gabor frame.

(e) If $\mathcal{G}(g, \Lambda)$ is a Riesz basis for $L^{2}\left(\mathbf{R}^{d}\right)$, then we say that it is a Gabor Riesz basis.

(f) If $\mathcal{G}(g, \Lambda)$ is a Riesz sequence in $L^{2}\left(\mathbf{R}^{d}\right)$, i.e., it is a Riesz basis for its closed linear span in $L^{2}\left(\mathbf{R}^{d}\right)$, then we say that it is a Gabor Riesz sequence.

We note that the order of translation and modulation in the definition of a Gabor system given by (1.1) can be interchanged if desired, since $M_{\xi} T_{x}=e^{2 \pi i x \cdot \xi} T_{x} M_{\xi}$, and multiplication by scalars of unit modulus does not affect the definitions given above.

For the case of a rectangular lattice Gabor frame $\mathcal{G}\left(g, \alpha \mathbf{Z}^{d} \times \beta \mathbf{Z}^{d}\right)$, it is easy to see that the frame operator $S$ commutes with the translation operators $T_{\alpha k}$ and the modulation operators $M_{\beta n}$ when $k, n \in \mathbf{Z}^{d}$. As a consequence, the canonical dual frame is another Gabor frame, namely, it is $\mathcal{G}\left(\tilde{g}, \alpha \mathbf{Z}^{d} \times \beta \mathbf{Z}^{d}\right)$ where $\tilde{g}=S^{-1} g$ is the canonical dual window. Similarly, the canonical Parseval frame is $\mathcal{G}\left(g^{\sharp}, \alpha \mathbf{Z}^{d} \times \beta \mathbf{Z}^{d}\right)$ where $g^{\sharp}=S^{-1 / 2} g$.

In general, the dual frame of an irregular Gabor frame need not itself be a Gabor frame, see Section 6.3 below.

\subsection{The STFT and the Modulation Spaces}

Time-frequency concentration is quantified by the norms of the modulation spaces. The modulation spaces were invented and extensively investigated by Feichtinger, with some of the main references being [61, 64, 65, 67], [89, Chapters 11-13]. They are now recognized as the appropriate function spaces for time-frequency analysis, and occur naturally in mathematical problems involving time-frequency shifts $M_{\xi} T_{x}$. For an interesting personal historical account of the development of the modulation spaces, including the Feichtinger algebra in particular, see Feichtinger's recent article [63].

Definition/Facts 2 (STFT). The short-time Fourier transform (STFT) of a function or tempered distribution $f$ with respect to a window $g$ is

$$
V_{g} f(x, \xi)=\left\langle f, M_{\xi} T_{x} g\right\rangle=\int_{\mathbf{R}^{d}} f(t) e^{-2 \pi i \xi \cdot t} \overline{g(t-x)} d t, \quad(x, \xi) \in \mathbf{R}^{2 d} .
$$

The STFT is a time-frequency or phase-space representation of $f$. If $g$ is restricted to lie in the Schwartz class $\mathcal{S}\left(\mathbf{R}^{d}\right)$ then $V_{g} f$ is a uniformly continuous function on $\mathbf{R}^{2 d}$ for any tempered distribution $f \in \mathcal{S}^{\prime}\left(\mathbf{R}^{d}\right)$. If $g \in L^{2}\left(\mathbf{R}^{d}\right)$ then $f \mapsto V_{g} f$ is a multiple of an isometry of $L^{2}\left(\mathbf{R}^{d}\right)$ into $L^{2}\left(\mathbf{R}^{2 d}\right)$. In particular, if $\|g\|_{2}=1$ then

$$
\|f\|_{2}=\left\|V_{g} f\right\|_{2}=\left(\int_{\mathbf{R}^{d}} \int_{\mathbf{R}^{d}}\left|V_{g} f(x, \xi)\right|^{2} d x d \xi\right)^{1 / 2} .
$$

Definition/Facts 3 (Modulation Spaces). Given a weight $w: \mathbf{R}^{d} \rightarrow(0, \infty)$, the modulation space $M_{w}^{p, q}\left(\mathbf{R}^{d}\right)$ consists of all tempered distributions $f$ for which the norm

$$
\|f\|_{M_{w}^{p, q}}=\left\|V_{g} f\right\|_{L_{w}^{p, q}}=\left(\int_{\mathbf{R}^{d}}\left(\int_{\mathbf{R}^{d}}\left|V_{g} f(x, \xi)\right|^{p} w(x, \xi)^{p} d x\right)^{q / p} d \xi\right)^{1 / q}
$$


is finite, with the usual adjustments if $p$ or $q$ is infinite. We write $M^{p, q}$ if $w=1$, and $M^{p}$ if $p=q$ and $w=1$. With some appropriate conditions on the weight, each $M_{w}^{p, q}$ is a Banach space, and its definition is independent of the choice of window $g$. That is, any nonzero function $g$ in the Schwartz class $\mathcal{S}$, or indeed any nonzero function in the modulation space $M^{1}$, may be used to define $M_{w}^{p, q}$, with each choice of $g$ determining the same space and an equivalent norm for the space.

The modulation space that will mostly appear in this article is the Feichtinger algebra $M^{1}$ (which is also often denoted by $S_{0}$ ). Roughly, membership in $M^{1}$ corresponds to the requirement that $g, \hat{g} \in L^{1}\left(\mathbf{R}^{d}\right)$. Precisely, it simply means that the STFT of $g$ is integrable over the time-frequency plane:

$$
\|f\|_{M^{1}}=\left\|V_{g} f\right\|_{1}=\int_{\mathbf{R}^{d}} \int_{\mathbf{R}^{d}}\left|V_{g} f(x, \xi)\right| d x d \xi .
$$

A fundamental fact proved by Feichtinger and Gröchenig is that Gabor frames with windows in $M^{1}$ (or its weighted counterparts) yield frame expansions valid beyond the Hilbert space setting. Specifically, for the unweighted case $(w=1)$ we have the following, see [89, Chapters 11-13] for details.

Theorem 1. Assume that $\mathcal{G}\left(g, \alpha \mathbf{Z}^{d} \times \beta \mathbf{Z}^{d}\right)$ is a frame for $L^{2}\left(\mathbf{R}^{d}\right)$, with window $g \in$ $M^{1}\left(\mathbf{R}^{d}\right)$. Then the following statements hold.

(a) The dual window $\tilde{g}$ belongs to $M^{1}\left(\mathbf{R}^{d}\right)$.

(b) For every $1 \leq p, q \leq \infty$ we have for all $f \in M^{p, q}\left(\mathbf{R}^{d}\right)$ that

$$
f=\sum_{k, n \in \mathbf{Z}^{d}}\left\langle f, M_{\beta n} T_{\alpha k} \tilde{g}\right\rangle M_{\beta n} T_{\alpha k} g=\sum_{k, n \in \mathbf{Z}^{d}}\left\langle f, M_{\beta n} T_{\alpha k} g\right\rangle M_{\beta n} T_{\alpha k} \tilde{g},
$$

where these series converge unconditionally in the norm of $M^{p, q}\left(\mathbf{R}^{d}\right)$ (weak ${ }^{*}$ convergence if $p=\infty$ or $q=\infty$ ).

(c) For every $1 \leq p, q \leq \infty$ the Gabor frame coefficients provide an equivalent norm for the modulation space $M^{p, q}\left(\mathbf{R}^{d}\right)$, i.e.,

$$
\|f\|_{M^{p, q}}=\left(\sum_{n \in \mathbf{Z}^{d}}\left(\sum_{k \in \mathbf{Z}^{d}}\left|\left\langle f, M_{\beta n} T_{\alpha k} g\right\rangle\right|^{p}\right)^{q / p}\right)^{1 / q}
$$

is an equivalent norm for $M^{p, q}\left(\mathbf{R}^{d}\right)$.

Thus, $\mathcal{G}\left(g, \alpha \mathbf{Z}^{d} \times \beta \mathbf{Z}^{d}\right)$ is a Banach frame for $M^{p, q}$ in the sense of $[86,36]$-but even more importantly, the frame expansions are simultaneously convergent in the entire range of modulation spaces $M^{p, q}$.

The fact that if $g \in M^{1}$ then $\tilde{g} \in M^{1}$ as well was proved for the case that $\alpha \beta$ is rational by by Feichtinger and Gröchenig in [67]. Gröchenig and Leinert [97] proved this result for arbitrary $\alpha \beta$, using deep results on symmetric Banach algebras (see [74] for an exposition of this proof). A different proof based on the concept of localized frames was given in [13]. That proof also extends to irregular Gabor frames, whose index set is not a lattice, and whose dual frame will not itself be a Gabor frame.

\subsection{Wiener Amalgam Spaces}

An amalgam space combines a local criterion for membership with a global criterion. The first amalgam spaces were introduced by Wiener in his study of generalized harmonic analy- 
sis $[186,187]$. A comprehensive general theory of amalgam spaces on locally compact groups was introduced and extensively studied by Feichtinger, e.g., [60, 64, 65]. For an expository introduction to Wiener amalgams on $\mathbf{R}$ with extensive references to the original literature, we refer to [105]. For our purposes, we will need the following particular amalgam spaces.

Definition 2. Let $Q=[0,1]^{d}$. Given $1 \leq p, q \leq \infty$, the Wiener amalgam space $W\left(L^{p}, \ell^{q}\right)=W\left(L^{p}, \ell^{q}\right)\left(\mathbf{R}^{d}\right)$ consists of all functions $F: \mathbf{R}^{d} \rightarrow \mathbf{C}$ for which

$$
\|F\|_{W\left(L^{p}, \ell^{q}\right)}=\left(\sum_{k \in \mathbf{Z}^{d}}\left\|F \cdot \chi_{Q+k}\right\|_{p}^{q}\right)^{1 / q}<\infty
$$

with the usual adjustment if $q=\infty$.

$W\left(L^{p}, \ell^{q}\right)$ is a Banach space, and the space

$$
W\left(\mathcal{C}, \ell^{q}\right)=\left\{F \in W\left(L^{\infty}, \ell^{q}\right): F \text { is continuous }\right\}
$$

is a closed subspace of $W\left(L^{\infty}, \ell^{q}\right)$. We have the inclusions

$$
L^{p}\left(\mathbf{R}^{d}\right)=W\left(L^{p}, \ell^{p}\right) \subset W\left(L^{1}, \ell^{p}\right) .
$$

We also have the following useful inclusions relating the Feichtinger algebra and the amalgam spaces:

$$
M^{1}\left(\mathbf{R}^{d}\right) \subset W\left(\mathcal{C}, \ell^{1}\right) \subset C_{0}\left(\mathbf{R}^{d}\right) \cap L^{1}\left(\mathbf{R}^{d}\right) .
$$

One importance use of the Wiener amalgam spaces in Gabor theory is the following result that a Gabor system using any window in $W\left(L^{\infty}, \ell^{1}\right)$ will automatically be a Bessel sequence, see [89, Proposition 6.2.2] (and compare Proposition 12 below for an analogous result for irregular Gabor systems). This fact is related to the Walnut representation that is discussed further in Section 3.9.

Proposition 1. If $g \in W\left(L^{\infty}, \ell^{1}\right)$ and $\alpha, \beta>0$, then $\mathcal{G}\left(g, \alpha \mathbf{Z}^{d} \times \beta \mathbf{Z}^{d}\right)$ is a Bessel sequence in $L^{2}\left(\mathbf{R}^{d}\right)$.

To point out one interesting but easy consequence, we obtain the following perturbation theorem [35, Theorem 3.2].

Corollary 1. Assume $\mathcal{G}\left(g, \alpha \mathbf{Z}^{d} \times \beta \mathbf{Z}^{d}\right)$ is a Gabor frame for $L^{2}\left(\mathbf{R}^{d}\right)$ with frame bounds $A, B$. If $h \in L^{2}\left(\mathbf{R}^{d}\right)$ and $\|g-h\|_{W\left(L^{\infty}, \ell^{1}\right)}$ is small enough, then $\mathcal{G}\left(h, \alpha \mathbf{Z}^{d} \times \beta \mathbf{Z}^{d}\right)$ is a frame for $L^{2}\left(\mathbf{R}^{d}\right)$.

Proof. The Bessel bound for $\mathcal{G}\left(g-h, \alpha \mathbf{Z}^{d} \times \beta \mathbf{Z}^{d}\right)$ is directly related to $\|g-h\|_{W\left(L^{\infty}, \ell^{1}\right)}$, see [35]. Therefore, if $\|g-h\|_{W\left(L^{\infty}, \ell^{1}\right)}$ is small enough then $\mathcal{G}\left(g-h, \alpha \mathbf{Z}^{d} \times \beta \mathbf{Z}^{d}\right)$ will have a Bessel bound $R<A$. Hence for $f \in L^{2}\left(\mathbf{R}^{d}\right)$ we have

$$
\begin{aligned}
\left(\sum_{k, n \in \mathbf{Z}^{d}}\right. & \left.\left|\left\langle f, M_{\beta n} T_{\alpha k} h\right\rangle\right|^{2}\right)^{1 / 2} \\
& \geq\left(\sum_{k, n \in \mathbf{Z}^{d}}\left|\left\langle f, M_{\beta n} T_{\alpha k} g\right\rangle\right|^{2}\right)^{1 / 2}-\left(\sum_{k, n \in \mathbf{Z}^{d}}\left|\left\langle f, M_{\beta n} T_{\alpha k}(g-h)\right\rangle\right|^{2}\right)^{1 / 2} \\
& \geq A^{1 / 2}\|f\|_{2}-R^{1 / 2}\|f\|_{2} .
\end{aligned}
$$


Thus $\mathcal{G}\left(h, \alpha \mathbf{Z}^{d} \times \beta \mathbf{Z}^{d}\right)$ has a lower frame bound of $\left(A^{1 / 2}-R^{1 / 2}\right)^{2}>0$, and the upper frame bound is similar.

Another important fact is that the STFT defined in (2.4) with respect to a window from $M^{1}$ belongs to an amalgam space on $\mathbf{R}^{2 d}$, as follows [89, Theorem 12.2.1].

Theorem 2. If $g \in M^{1}\left(\mathbf{R}^{d}\right)$ and $1 \leq p \leq \infty$, then $V_{g} f \in W\left(\mathcal{C}, \ell^{p}\right)\left(\mathbf{R}^{2 d}\right)$ for all $f \in M^{p}\left(\mathbf{R}^{d}\right)$. Further, there exists a $C>0$ such that

$$
\forall f \in M^{p}\left(\mathbf{R}^{d}\right), \quad\left\|V_{g} f\right\|_{W\left(\mathcal{C}, \ell^{p}\right)} \leq C\|f\|_{M^{p}} .
$$

In particular, for any $f \in L^{2}\left(\mathbf{R}^{d}\right)=M^{2}\left(\mathbf{R}^{d}\right)$ we have $V_{g} f \in W\left(\mathcal{C}, \ell^{2}\right)\left(\mathbf{R}^{2 d}\right)$.

\section{Rectangular Lattice Gabor Systems}

Now we turn to the Density Theorem itself. We begin with rectangular lattices in dimension $d=1$. In this case, the Density Theorem is as follows.

Theorem 3 (Density Theorem for Rectangular Lattices). Let $g \in L^{2}(\mathbf{R})$ and let $\Lambda=$ $\alpha \mathbf{Z} \times \beta \mathbf{Z}$ where $\alpha, \beta>0$. Then the following statements hold.

(a) If $\alpha \beta>1$, then $\mathcal{G}(g, \alpha \mathbf{Z} \times \beta \mathbf{Z})$ is incomplete in $L^{2}(\mathbf{R})$.

(b) If $\mathcal{G}(g, \alpha \mathbf{Z} \times \beta \mathbf{Z})$ is a frame for $L^{2}(\mathbf{R})$, then $0<\alpha \beta \leq 1$.

(c) $\mathcal{G}(g, \alpha \mathbf{Z} \times \beta \mathbf{Z})$ is a Riesz basis for $L^{2}(\mathbf{R})$ if and only if it is a frame for $L^{2}(\mathbf{R})$ and $\alpha \beta=1$.

(d) If $\mathcal{G}(g, \alpha \mathbf{Z} \times \beta \mathbf{Z})$ is a Riesz sequence in $L^{2}(\mathbf{R})$, then $\alpha \beta \geq 1$.

Note that the necessary conditions in Theorem 3 depend only on the lattice $\alpha \mathbf{Z} \times \beta \mathbf{Z}$ and not on $g$. Further, only the value of the product $\alpha \beta$ is relevant because by applying the unitary dilation operator $D_{r} g(t)=r^{1 / 2} g(r t)$, we see that $\mathcal{G}(g, \alpha \mathbf{Z} \times \beta \mathbf{Z})$ is complete, a frame, a Riesz basis, or a Riesz sequence if and only if the same is true of $\mathcal{G}\left(D_{r} g, \frac{\alpha}{r} \mathbf{Z} \times r \beta \mathbf{Z}\right)$. The dilation operator is one example of a metaplectic transform, discussed in more detail in Section 4.1 below.

For a textbook account of the Density Theorem, see [89, Section 7.5]. As we will see in Section 5, the upper and lower Beurling densities of the lattice $\Lambda=\alpha \mathbf{Z} \times \beta \mathbf{Z}$ are $D^{+}(\Lambda)=D^{-}(\Lambda)=\frac{1}{\alpha \beta}$. Thus $\frac{1}{\alpha \beta}$ is the density of the lattice. The density $\frac{1}{\alpha \beta}=1$ is sometimes called the critical density or the Nyquist density.

We will discuss each part of the Density Theorem in turn in the following sections.

\subsection{Incompleteness}

Part (a) of Theorem 3 was proved for arbitrary values of $\alpha \beta$ by Baggett [6], and for the case that the product $\alpha \beta$ is rational by Daubechies [48], with both results appearing in 1990. The operators $M_{\beta n} T_{\alpha k}$ corresponding to the rectangular lattice $\alpha \mathbf{Z} \times \beta \mathbf{Z}$ generate a von Neumann algebra, and Baggett's proof made use of this; specifically, he used the representation theory of the discrete Heisenberg group. Daubechies' proof relied on the Zak transform, which is another "algebraic" tool in the sense that it is dependent on the fact that the index set is a rectangular lattice. The Zak transform is defined precisely in Section 3.7. For an exposition of Baggett's operator-theoretic proof, see [74]. 
Daubechies also noted in [48] that a proof for general $\alpha \beta$ can be inferred from results of Rieffel [159] on coupling constants of $C^{*}$-algebras. ${ }^{1}$ Another proof of part (a) based on von Neumann algebras was given by Daubechies, H. Landau, and Z. Landau in [54].

A different proof of part (a) was given by Ramanathan and Steger in [158]. Their proof did not use operator theory or the machinery of von Neumann algebras but instead was based on their proof that Gabor frames satisfy a certain Homogeneous Approximation Property (HAP). While both regular and irregular Gabor frames satisfy this HAP, the extra algebraic structure in the lattice case allowed Ramanathan and Steger to prove that a rectangular lattice Gabor system is incomplete when $\alpha \beta>1$, whereas for irregular frames they proved only that the Gabor system is not a frame when the density of $\Lambda$ is too small. The HAP is discussed in more detail in Section 5.3 below.

Gabardo and Han [78] used their operator-theoretic approach to derive a proof of part (a) that also extends to certain more general non-Gabor settings, namely, what they call group-like unitary systems. Bownik and Rzeszotnik gave a proof of part (c) in [27] based on the use of the spectral function rather than the machinery of von Neumann algebras.

\section{$3.2 \quad$ Frames}

Since every frame is complete, part (b) of the Density Theorem is of course a consequence of part (a), but we state it separately to emphasize the contrast with the case of irregular Gabor systems (see Theorem 13 below).

For the rectangular lattice case, a "simple" proof of part (b) was given by Janssen [121]. This proof relies on the algebraic structure of the rectangular lattice $\alpha \mathbf{Z} \times \beta \mathbf{Z}$ and the remarkable Wexler-Raz biorthogonality relations for Gabor frames $\mathcal{G}(g, \alpha \mathbf{Z} \times \beta \mathbf{Z})$. A special case of the Wexler-Raz relations is the following.

Theorem 4 (Wexler-Raz Biorthogonality Relations). Let $g \in L^{2}(\mathbf{R})$ and $\alpha, \beta>0$ be such that $\mathcal{G}(g, \alpha \mathbf{Z} \times \beta \mathbf{Z})$ is a frame for $L^{2}(\mathbf{R})$. Let $\mathcal{G}(\tilde{g}, \alpha \mathbf{Z} \times \beta \mathbf{Z})$ be the canonical dual frame. Then $\mathcal{G}\left(g, \frac{1}{\beta} \mathbf{Z} \times \frac{1}{\alpha} \mathbf{Z}\right)$ and $\mathcal{G}\left(\tilde{g}, \frac{1}{\beta} \mathbf{Z} \times \frac{1}{\alpha} \mathbf{Z}\right)$ are biorthogonal, specifically,

$$
\frac{1}{\alpha \beta}\left\langle M_{\frac{n}{\alpha}} T_{\frac{k}{\beta}} g, M_{\frac{n^{\prime}}{\alpha}} T_{\frac{k^{\prime}}{\beta}} \tilde{g}\right\rangle=\delta_{k k^{\prime}} \delta_{n n^{\prime}} .
$$

The Wexler-Raz relations can actually be formulated much more generally, e.g., for duals other than the canonical dual, and even the frame hypothesis can be relaxed. In fact, the Wexler-Raz relations can be used to characterize all the dual windows, see [72], [89, Theorem 7.3.1]. The Wexler-Raz relations were introduced in [185]; for more details see [121, 125, 127], the exposition in [89, Section 7.5], and the rigorous proofs in [122, 54].

In spirit, the Wexler-Raz relations are an infinite-dimensional version of the familiar finite-dimensional fact that if the columns of an $m \times n$ complex matrix $A$ are linearly independent, then the rows of $A$ must span $\mathbf{C}^{n}$, and conversely. An additional interesting point is the appearance in the Wexler-Raz relations of the adjoint lattice $\frac{1}{\beta} \mathbf{Z} \times \frac{1}{\alpha} \mathbf{Z}$. The adjoint of an arbitrary lattice will play an important role in the discussion in Section 4.4 below.

If we accept the Wexler-Raz relations, then we can easily derive parts of the Density Theorem, as follows. Suppose that $\mathcal{G}(g, \alpha \mathbf{Z} \times \beta \mathbf{Z})$ is a frame for $L^{2}(\mathbf{R})$, and let $S$ be the

\footnotetext{
${ }^{1}$ For this reason, part (a) is sometimes credited to Rieffel. However, no part of the Density Theorem is explicitly stated in [159].
} 
corresponding frame operator. Then the dual window $\tilde{g}$ is given by $\tilde{g}=S^{-1} g$, and so Equation (3.1) implies that

$$
\alpha \beta=\langle g, \tilde{g}\rangle=\left\langle g, S^{-1} g\right\rangle=\left\langle S^{-1 / 2} g, S^{-1 / 2} g\right\rangle=\left\|S^{-1 / 2} g\right\|_{2}^{2} .
$$

However, $\mathcal{G}\left(S^{-1 / 2} g, \alpha \mathbf{Z} \times \beta \mathbf{Z}\right)$ is the canonical Parseval frame associated with $\mathcal{G}(g, \alpha \mathbf{Z} \times \beta \mathbf{Z})$, and therefore it has frame bounds $A=B=1$. Since $B^{1 / 2}$ is an upper bound to the norms of a frame's elements and since $g \neq 0$, we must have $0<\left\|S^{-1 / 2} g\right\|_{2} \leq 1$. Therefore $0<\alpha \beta \leq 1$. This proves part (b) of Theorem 3 .

On the other hand, if $\mathcal{G}(g, \alpha \mathbf{Z} \times \beta \mathbf{Z})$ is a Riesz basis for $L^{2}(\mathbf{R})$ then $\mathcal{G}\left(S^{-1 / 2} g, \alpha \mathbf{Z} \times \beta \mathbf{Z}\right)$ is both a Parseval frame and a basis, and hence is an orthonormal basis for $L^{2}(\mathbf{R})$. Therefore $\alpha \beta=\left\|S^{-1 / 2} g\right\|_{2}=1$, which proves part (c) of Theorem 3 .

Part (d) of Theorem 3, dealing with Riesz sequences, is also related to the WexlerRaz relations, and more specifically to a result known as the Duality Principle. We will discuss the Duality Principle in Section 3.4 below, and show there how to derive part (d) of Theorem 3 from it.

In addition to these remarks, we note that Balan gave a direct proof of part (b) of the Density Theorem in [8] and furthermore extended the Density Theorem to Gabor superframes for $L^{2}(\mathbf{R}) \times \cdots \times L^{2}(\mathbf{R})$ (superframes are quite distinct from Gabor frames for $L^{2}(\mathbf{R})$ with multiple generators, i.e., frames of the form $\left.\mathcal{G}\left(g_{1}, \Lambda_{1}\right) \cup \cdots \cup \mathcal{G}\left(g_{r}, \Lambda_{r}\right)\right)$. In 1999, Janssen gave a "simple" (one page) proof of parts (b) and (d) that does not use the Wexler-Raz relations. This proof is available electronically in [130] (note that what we call a Gabor Riesz sequence is there referred to as a Riesz-Gabor basis); see also [122] and [125]. Another straightforward proof of part (b) was given by Han and Wang in [101]; furthermore, their proof extends to certain non-rectangular lattices in higher dimensions.

\subsection{Riesz Bases}

In part (c) of the Density Theorem, the fact that if $\mathcal{G}(g, \alpha \mathbf{Z} \times \beta \mathbf{Z})$ is a frame and $\alpha \beta=1$ then $\mathcal{G}(g, \alpha \mathbf{Z} \times \beta \mathbf{Z})$ must be a Riesz basis is easy to prove using the Zak transform (see the details in Section 3.7 below). In the 1989 research-tutorial article [113], it was stated as a fact that if $\mathcal{G}(g, \alpha \mathbf{Z} \times \beta \mathbf{Z})$ is a Riesz basis then $\alpha \beta=1$. However, while that was folklore at the time, no proof actually existed then. The first proof was given by Ramanathan and Steger in [158], along with a much more general result for irregular Gabor frames (see Theorem 13 below). As pointed out above, there are today "straightforward" proofs of part (c).

Unfortunately, there do not exist any "good" Gabor Riesz bases $\mathcal{G}(g, \alpha \mathbf{Z} \times \beta \mathbf{Z})$. The Balian-Low Theorem, which we discuss in Section 3.7 below, implies that the window $g$ of a Gabor Riesz basis $\mathcal{G}(g, \alpha \mathbf{Z} \times \beta \mathbf{Z})$ must be poorly localized in the time-frequency plane. Consequently, redundant Gabor frames $(\alpha \beta<1)$ are most often used in practice.

\subsection{The Duality Principle}

The Wexler-Raz relations and parts (b), (c), and (d) of the Density Theorem are closely related to another fundamental principle for Gabor systems known as the Duality Principle. A restricted form of the Duality Principle is as follows.

Theorem 5 (Duality Principle). Let $g \in L^{2}(\mathbf{R})$ and let $\Lambda=\alpha \mathbf{Z} \times \beta \mathbf{Z}$ where $\alpha, \beta>0$. Then the following statements are equivalent. 
(a) $\mathcal{G}(g, \alpha \mathbf{Z} \times \beta \mathbf{Z})$ is a frame for $L^{2}(\mathbf{R})$, with frame bounds $A, B$.

(b) $\mathcal{G}\left(g, \frac{1}{\beta} \mathbf{Z} \times \frac{1}{\alpha} \mathbf{Z}\right)$ is a Riesz sequence in $L^{2}(\mathbf{R})$, with frame bounds $\alpha \beta A, \alpha \beta B$ (as a frame for its closed span).

The Duality Principle leads immediately to a proof of part (d) of Theorem 3. For, if we interchange the roles of $\alpha, \beta$ and $\frac{1}{\beta}, \frac{1}{\alpha}$ in Theorem 5, we see that if $\mathcal{G}(g, \alpha \mathbf{Z} \times \beta \mathbf{Z})$ is a Riesz sequence in $L^{2}(\mathbf{R})$, then $\mathcal{G}\left(g, \frac{1}{\beta} \mathbf{Z} \times \frac{1}{\alpha} \mathbf{Z}\right)$ must be a frame for $L^{2}(\mathbf{R})$. But then by Theorem 3(b), we must have $\frac{1}{\beta} \frac{1}{\alpha} \leq 1$, which implies $\alpha \beta \geq 1$.

Independent and essentially simultaneous proofs of Theorem 5 were published by Daubechies, H. Landau, and Z. Landau [54], Janssen [122], and Ron and Shen [162], ${ }^{2}$ and we also point out the related article [179] which contains the special case of the Theorem 5 corresponding to the assumption that $\mathcal{G}(g, \alpha \mathbf{Z} \times \beta \mathbf{Z})$ is a tight frame. Interestingly, the techniques used in these three article to prove the Duality Principle are completely different.

Theorem 5 is sometimes referred to as the Ron-Shen Duality Principle. Ron and Shen obtained some other equivalences in addition to those given in Theorem 5 , e.g., for the cases where $\mathcal{G}(g, \alpha \mathbf{Z} \times \beta \mathbf{Z})$ is assumed to be a a Bessel sequence or a frame sequence. Their formulation of the Duality Principle is also valid for lattice Gabor frames $\mathcal{G}(g, \Lambda)$ in higher dimensions where $\Lambda$ is separable, i.e., $\Lambda$ has the form $\Lambda=A\left(\mathbf{Z}^{d}\right) \times B\left(\mathbf{Z}^{d}\right)$ where $A, B$ are $d \times d$ invertible matrices. Lattices in higher dimensions will be discussed in more detail in Section 4.4.

For more on the Duality Principle see [125] and [89, Section 7.4]. Recent extensions of the Duality Principle beyond the Gabor setting appear in [38].

\subsection{Existence}

The Density Theorem only provides necessary conditions for existence, and these are clearly not sufficient in general. For example, $\mathcal{G}\left(\chi_{[0,1 / 2]}, \mathbf{Z}^{2}\right)$ is an orthogonal sequence in $L^{2}(\mathbf{R})$ but it is not complete, yet we do have $\alpha \beta=1$ for this system.

Daubechies [48, p. 979] proved that if $g \in L^{2}(\mathbf{R})$ and $\alpha, \beta>0$ are given, then in addition to the requirement $\alpha \beta \leq 1$, another basic necessary condition for $\mathcal{G}(g, \alpha \mathbf{Z} \times \beta \mathbf{Z})$ to be a frame is that there exist constants $C, D$ such that

$$
0<C \leq \sum_{k \in \mathbf{Z}}|g(t-\alpha k)|^{2} \leq D<\infty \text { a.e.; }
$$

see also [113, Proposition 4.1.4]. If $g$ is compactly supported with its support contained in an interval of length $1 / \beta$, then (3.2) is also sufficient for $\mathcal{G}(g, \alpha \mathbf{Z} \times \beta \mathbf{Z})$ to be a frame, cf. [51] or [113, Theorem 4.1.2]. In general, however, (3.2) is not a sufficient condition.

In the one-dimensional, rectangular lattice setting, the "Painless Nonorthogonal Expansions" of Daubechies, Grossmann, and Meyer [51] show that whenever $0<\alpha \beta \leq 1$, we can construct a $g \in L^{2}(\mathbf{R})$ supported in $[0,1 / \beta]$ such that (3.2) holds, and hence $\mathcal{G}(g, \alpha \mathbf{Z} \times \beta \mathbf{Z})$ forms a frame for $L^{2}(\mathbf{R})$. When $\alpha \beta<1$, we can even do this with $g \in C_{c}^{\infty}(\mathbf{R})$. For $\alpha \beta=1$, if we let $g$ be the characteristic function of the interval [0, $\alpha$, then $\mathcal{G}\left(g, \alpha \mathbf{Z} \times \frac{1}{\alpha} \mathbf{Z}\right)$

\footnotetext{
${ }^{2}$ Note that the notation in [162] differs from the notation of this article. In particular, what we call a "frame sequence" is called a "frame" in [162], and what we call a "frame" is there called a "fundamental frame." Additionally, what [162] terms a "Riesz basis" is what we call a "Riesz sequence", and an "orthonormal basis" in [162] is for us an "orthonormal sequence."
} 
is an orthonormal basis for $L^{2}(\mathbf{R})$. The fact that the construction allows much "nicer" functions when $\alpha \beta<1$ is again a reflection of the Balian-Low Theorem, which is discussed in more detail later.

Thus, the question of whether for each $\alpha, \beta>0$ there exists a $g \in L^{2}(\mathbf{R})$ such that $\mathcal{G}(g, \alpha \mathbf{Z} \times \beta \mathbf{Z})$ is a frame for $L^{2}(\mathbf{R})$ is easily answered - at least in the rectangular lattice setting that we are considering here. The analogous question for general lattices in higher dimensions is much more difficult, see Section 4.2 below.

On the other hand, the existence question becomes extremely difficult if we instead ask: Given $g \in L^{2}(\mathbf{R})$, for which $\alpha, \beta$ will $\mathcal{G}(g, \alpha \mathbf{Z} \times \beta \mathbf{Z})$ be a frame? Currently, there are only a few functions for which a complete answer is known. Of course, if $\mathcal{G}(g, \alpha \mathbf{Z} \times \beta \mathbf{Z})$ is a frame, then we can easily obtain infinitely many other related frames:

- $\mathcal{G}\left(M_{\omega} T_{x} g, \alpha \mathbf{Z} \times \beta \mathbf{Z}\right)$ is a frame for all $x, \omega \in \mathbf{R}$;

- $\mathcal{G}\left(D_{r} g, \frac{\alpha}{r} \mathbf{Z} \times r \beta \mathbf{Z}\right)$ is a frame for all $r>0$;

- $\mathcal{G}(\hat{g}, \beta \mathbf{Z} \times \alpha \mathbf{Z})$ is a frame, where $\hat{g}$ is the Fourier transform of $g$.

Aside from modifications such as these, the following windows are the only ones for which a complete characterization of frame properties is currently known, as far as we are aware at the time of writing (the functions are normalized so that their $L^{2}$-norm is 1 ).

(a) For the Gaussian function $\varphi(t)=2^{1 / 4} e^{-\pi t^{2}}$, Daubechies and Grossmann conjectured $^{3}$ [48, p. 981] that $\mathcal{G}(\varphi, \alpha \mathbf{Z} \times \beta \mathbf{Z})$ is a frame if and only if $0<\alpha \beta<1$ [50]. Evidence supporting this conjecture was given in [48], and the full conjecture was proved by Lyubarskii [150] and by Seip and Wallstén [164, 168] (see also the simple proof and additional references in [121]). The proof relies on the fact that the Bargmann transform of $f \in L^{2}(\mathbf{R})$ is an analytic function, and in fact the results of Lyubarskii and Seip-Wallstén are formulated in terms of the density of sets of sampling and interpolation in the Bargmann-Fock space (see Appendix A for a discussion of the relationship between Gabor systems and the Bargmann-Fock space). The Bargmann transform is closely related to the short-time Fourier transform $V_{\varphi} f$ using a Gaussian window, e.g., see [89, Section 3.4]. This characterization also extends to irregular Gabor frames generated by the Gaussian window, see Section 5.6 below.

At the critical density, $\mathcal{G}\left(\varphi, \alpha \mathbf{Z} \times \frac{1}{\alpha} \mathbf{Z}\right)$ is not a frame, and is overcomplete by exactly one element. If any single element is removed, the resulting system is exact, but is not a Schauder basis. This is proved in [73, p. 168], and is also related to results of Kazarian that if $g \in L^{2}[0,1]$ and $F \subset \mathbf{Z}$ is finite, then $\left\{g(x) e^{2 \pi i n x}\right\}_{n \in \mathbf{Z} \backslash F}$ can never be a Schauder basis for $L^{p}[0,1]$, see $[133,134]$. Convergence and summability of Gabor expansions based on modifications of $\mathcal{G}\left(\varphi, \alpha \mathbf{Z} \times \frac{1}{\alpha} \mathbf{Z}\right)$ are extensively studied in [151] (see also [118]).

(b) Janssen and Strohmer [131] proved that for the hyperbolic secant window $g(t)=$ $(\pi / 2)^{1 / 2}(\cosh \pi t)^{-1}, \mathcal{G}(g, \alpha \mathbf{Z} \times \beta \mathbf{Z})$ is a frame if and only if $0<\alpha \beta<1$. The proof for this and the next two examples relies on the use of the Zak transform, which is defined in Section 3.7.

\footnotetext{
${ }^{3}$ Daubechies further conjectured in [48] that if $g \in L^{2}(\mathbf{R})$ is such that both $g$ and $\hat{g}$ are everywhere positive, then $\mathcal{G}(\varphi, \alpha \mathbf{Z} \times \beta \mathbf{Z})$ will be a frame if and only if $0<\alpha \beta<1$. However, this was shown to be false in [124].
} 
(c) The two-sided exponential $g(t)=e^{-|t|}$ generates a frame if and only if $0<\alpha \beta<1$ [129]. Taking the Fourier transform, we see that the same is true for the window $g(t)=2 /\left(1+4 \pi^{2} t^{2}\right)$.

(d) The one-sided exponential $g(t)=2^{1 / 2} e^{-t} \chi_{[0, \infty)}(t)$ generates a frame if and only if $0<\alpha \beta \leq 1$. In particular, if $\alpha \beta=1$ then this system is a Riesz basis for $L^{2}(\mathbf{R})$. Taking the Fourier transform, we see that the same is true for the window $g(t)=2^{1 / 2} /(1+2 \pi i t)$.

The characterization problem is very difficult even for the seemingly "simple" case of a characteristic function. The precise set of $(\alpha, \beta)$ for which $\mathcal{G}\left(\chi_{[0,1]}, \alpha \mathbf{Z} \times \beta \mathbf{Z}\right)$ is a frame for $L^{2}(\mathbf{R})$ is not known, and, surprisingly, it appears to be an extremely complicated set, called "Janssen's tie" [128]. Also, it has been shown that a solution to the problem of characterizing those sets $E \subset \mathbf{R}$ such that $\mathcal{G}\left(\chi_{E}, \mathbf{Z}^{2}\right)$ is a frame would imply a solution to a longstanding open problem of Littlewood [37].

On the other hand, if $g$ is reasonably "nice" (specifically, $g$ belongs to the Wiener amalgam space $W\left(L^{\infty}, \ell^{1}\right)$ defined in Section 2.4), and if $g$ fulfills the necessary condition (3.2) for some $\alpha$, then for all sufficiently small $\beta$ it will be the case that $\mathcal{G}(g, \alpha \mathbf{Z} \times \beta \mathbf{Z})$ will be a frame. This was proved by Walnut [181] (with a proof also appearing in [113]), extending a weaker result proved by Daubechies in [48]; see also [89, Theorem 6.5.1].

It is not true in general that for an arbitrary nonzero $g \in L^{2}(\mathbf{R})$, the Gabor system $\mathcal{G}(g, \alpha \mathbf{Z} \times \beta \mathbf{Z})$ will be a frame if $\alpha, \beta$ are chosen small enough [69]. However, if we impose further restrictions (namely that $g$ lie in the modulation space $M^{1}$, which is a subspace of $\left.W\left(L^{\infty}, \ell^{1}\right)\right)$, then this is indeed the case. This follows from the abstract coorbit theory of Feichtinger and Gröchenig, see [64, Theorem 6.1], [86, Theorem 5.2], [66]. This result was also proved by Bittner and Chui [25] assuming the weaker hypotheses that $g, \hat{g} \in W\left(L^{\infty}, \ell^{1}\right)$; see also [89, p. 126].

Feichtinger and Kaiblinger proved in [70] that if $g \in M^{1}$, then the set of $(\alpha, \beta)$ such that $\mathcal{G}(g, \alpha \mathbf{Z} \times \beta \mathbf{Z})$ is a frame is an open (but not necessarily connected!) subset of $\mathbf{R}^{2}$, compare also [72, Theorem 3.6.9]. This fact is especially interesting because as we vary $(\alpha, \beta)$ from $\left(\alpha_{1}, \beta_{1}\right)$ to $\left(\alpha_{2}, \beta_{2}\right)$, the corresponding indices in the sets $\alpha_{1} \mathbf{Z} \times \beta_{1} \mathbf{Z}$ and $\alpha_{2} \mathbf{Z} \times \beta_{2} \mathbf{Z}$ become arbitrarily far apart; thus this result is not just a "local perturbation" theorem, as is considered in such article as $[180,59,44,40,173]$.

\subsection{Excess}

If $\mathcal{G}(g, \alpha \mathbf{Z} \times \beta \mathbf{Z})$ is a Gabor frame and $\alpha \beta<1$, then we know by the Density Theorem that this frame is not a Riesz basis. Duffin and Schaeffer [57] showed in their original frame article that any frame that is not a Riesz basis is overcomplete in the sense that at least finitely many elements may be removed yet still be a frame. A crude measure of this overcompleteness or redundancy is the excess of the frame, which is the supremum of the cardinality of those subsets which can be removed yet still leave a complete set. It was shown in [10] that every Gabor frame $\mathcal{G}(g, \alpha \mathbf{Z} \times \beta \mathbf{Z})$ with $\alpha \beta<1$ has infinite excess. Further, it was shown there that there exists an infinite subset $J$ of $\alpha \mathbf{Z} \times \beta \mathbf{Z}$ such that $\mathcal{G}(g,(\alpha \mathbf{Z} \times \beta \mathbf{Z}) \backslash J)$ is still a frame (this is not a consequence of infinite excess). However, the subset constructed in [10] is extremely sparse (specifically, it has Beurling density zero). In [13] it is shown that if $g$ has some concentration in the time-frequency plane (specifically, $g$ lies in the modulation space $M^{1}$ ), then a subset of positive density may be removed yet still leave a frame (see Section 6.2). 


\subsection{The Balian-Low Theorem}

The Density Theorem implies that a Gabor system $\mathcal{G}(g, \alpha \mathbf{Z} \times \beta \mathbf{Z})$ can form a Riesz basis for $L^{2}(\mathbf{R})$ only at the critical density (i.e., $\alpha \beta=1$ ). However, the Balian-Low Theorem (BLT), which we will discuss in this section, implies that the window $g$ of any Gabor Riesz basis $\mathcal{G}(g, \alpha \mathbf{Z} \times \beta \mathbf{Z})$ must either not be smooth or must decay poorly at infinity, or, put in another terminology, $g$ must have poor joint time-frequency localization in the time-frequency plane.

We will prove one variant of the BLT in this section and discuss the Zak transform, which is a fundamental tool for analyzing Gabor frames, especially at the critical density. For historical comments and additional discussion of the BLT we refer to [23].

Gröchenig [89, p. 148] states that the Zak transform was first introduced by Gelfand [81]. As with many useful notions, it has been rediscovered many times and goes by a variety of names. Weil [184] defined a Zak transform for locally compact abelian groups, and this transform is often called the Weil-Brezin map in representation theory and abstract harmonic analysis, e.g., [163, 4]. Zak rediscovered this transform, which he called the $k$ $q$ transform, in his work on quantum mechanics, e.g., [190, 5]. The terminology "Zak transform" has become customary in applied mathematics and signal processing. For more information, we refer to Janssen's influential article [119] and survey [120], or Gröchenig's text [89, Chapter 8].

One definition of the Zak transform on $L^{2}(\mathbf{R})$ is as follows. For simplicity, we will consider only the case $\alpha=\beta=1$ (which we can always reduce to whenever $\alpha \beta=1$ by making a change of variables). Let $\chi=\chi_{[0,1)}$ be the characteristic function of the interval $[0,1)$. Then the Gabor system $\mathcal{G}\left(\chi, \mathbf{Z}^{2}\right)=\left\{M_{n} T_{k} \chi\right\}_{k, n \in \mathbf{Z}}$ is an orthonormal basis for $L^{2}(\mathbf{R})$. Now consider

$$
\mathcal{E}=\left\{e_{n k}\right\}_{k, n \in \mathbf{Z}}, \quad \text { where } e_{n k}(t, \omega)=e^{2 \pi i n t} e^{-2 \pi i k \omega},
$$

which forms an orthonormal basis for $L^{2}\left([0,1)^{2}\right)$. The Zak transform is the unitary map $Z: L^{2}(\mathbf{R}) \rightarrow L^{2}\left([0,1)^{2}\right)$ defined by the condition that $Z$ map the orthonormal basis $\mathcal{G}\left(\chi, \mathbf{Z}^{2}\right)$ to the orthonormal basis $\mathcal{E}$. That is, $Z$ is the unique extension to $L^{2}(\mathbf{R})$ of the rule

$$
Z\left(M_{n} T_{k} \chi\right)=e_{n k}, \quad k, n \in \mathbf{Z} .
$$

Explicitly, it can be shown that for $f \in L^{2}(\mathbf{R})$,

$$
Z f(t, \omega)=\sum_{k \in \mathbf{Z}} f(t-k) e^{2 \pi i k \omega}, \quad(t, \omega) \in[0,1)^{2},
$$

where this series converges in the norm of $L^{2}\left([0,1)^{2}\right)$. The important fact for us is that for a general Gabor system $\mathcal{G}\left(g, \mathbf{Z}^{2}\right)$ with $\alpha=\beta=1$, we have

$$
Z\left(M_{k} T_{n} g\right)(t, \omega)=\left(e_{n k} \cdot Z g\right)(t, \omega)=e_{n k}(t, \omega) Z g(t, \omega) .
$$

Thus, the Zak transform diagonalizes time-frequency shifts. Since $Z$ is unitary, $\mathcal{G}\left(g, \mathbf{Z}^{2}\right)$ is therefore complete, a frame, a Riesz basis, or a Riesz sequence in $L^{2}(\mathbf{R})$ if and only if the same is true of $\left\{e_{n k} \cdot Z g\right\}_{k, n \in \mathbf{Z}}$ in $L^{2}\left([0,1)^{2}\right)$. But since this latter system has the form of an orthonormal basis with each element multiplied by the single function $Z g$, it is easy to prove the following (e.g., see [113, Theorem 4.3.3]).

Proposition 2. Let $g \in L^{2}(\mathbf{R})$ be given, and set $\alpha=\beta=1$. Then the following statements hold. 
(a) $\mathcal{G}\left(g, \mathbf{Z}^{2}\right)$ is complete in $L^{2}(\mathbf{R})$ if and only if $Z g \neq 0$ a.e.

(b) $\mathcal{G}\left(g, \mathbf{Z}^{2}\right)$ is minimal if and only if $1 / Z g \in L^{2}\left([0,1)^{2}\right)$. In this case, $\mathcal{G}\left(g, \mathbf{Z}^{2}\right)$ is exact.

(c) The following statements are equivalent.

(i) $\mathcal{G}\left(g, \mathbf{Z}^{2}\right)$ is a frame for $L^{2}(\mathbf{R})$.

(ii) There exist $A, B>0$ such that $A \leq|Z g|^{2} \leq B$ a.e.

(iii) $\mathcal{G}\left(g, \mathbf{Z}^{2}\right)$ is a Riesz basis for $L^{2}(\mathbf{R})$.

In case these hold, the dual window $\tilde{g}$ satisfies $Z \tilde{g}=1 / \overline{Z g}$.

We can derive some further interesting results from this. Once $Z f(t, \omega)$ is defined on the domain $[0,1)^{2}$, Equation (3.3) implies that the domain of $Z f(t, \omega)$ naturally extends to all of $\mathbf{R}^{2}$ and that $Z f$ satisfies the quasiperiodicity relations

$$
\begin{aligned}
& Z f(t, \omega+n)=Z f(t, \omega), \\
& Z f(t+n, \omega)=e^{2 \pi i n \omega} Z f(t, \omega),
\end{aligned}
$$

for $n \in \mathbf{Z}$. Consequently, $|Z f(t, \omega)|$ is 1-periodic in each variable. Yet $Z f(t, \omega)$ is not itself 1-periodic, and the behavior of a quasiperiodic function is quite different than that of a periodic function. In particular, we have the following fact.

Proposition 3. If $f \in L^{2}(\mathbf{R})$ and $Z f$ is continuous on $\mathbf{R}^{2}$, then $Z f$ has a zero in $[0,1)^{2}$.

While seemingly mysterious at first glance, Janssen gives an elegant demonstration in [130] of how Proposition 3 follows naturally from the definition of quasiperiodicity. See also the extension of Proposition 3 to locally compact abelian groups in [132].

Comparing Proposition 3 to Proposition 2(c), we see that if $Z g$ is continuous, then $\mathcal{G}\left(g, \mathbf{Z}^{2}\right)$ cannot be a frame or Riesz basis for $L^{2}(\mathbf{R})$. Yet $Z g$ is continuous for most "nice" functions. In particular, this is the case for all functions contained in the Wiener amalgam space $W\left(\mathcal{C}, \ell^{1}\right)$ defined in Section 2.4.

Theorem 6 (Amalgam Balian-Low Theorem). Choose $g \in L^{2}(\mathbf{R})$ and set $\alpha=\beta=1$. If $\mathcal{G}\left(g, \mathbf{Z}^{2}\right)$ is a Riesz basis for $L^{2}(\mathbf{R})$, then $g, \hat{g} \notin W\left(\mathcal{C}, \ell^{1}\right)$.

Proof. If $g \in W\left(\mathcal{C}, L^{1}\right)$, then the series defining $Z g$ in (3.3) converges not only in $L^{2}$ norm, but uniformly on compact subsets of $\mathbf{R}^{2}$. As each term in the series is continuous on $\mathbf{R}^{2}$, we conclude that $Z g$ is continuous on $\mathbf{R}^{2}$, and hence $\mathcal{G}\left(g, \mathbf{Z}^{2}\right)$ cannot be a Riesz basis. The same argument applies to $\hat{g}$ since the Fourier transform is a unitary transform that maps $\mathcal{G}\left(g, \mathbf{Z}^{2}\right)$ onto $\mathcal{G}\left(\hat{g}, \mathbf{Z}^{2}\right)$.

The Amalgam Balian-Low Theorem was first proved in [103, Corollary 7.5.3] and published in [23]. It is so-named because it is qualitatively similar to the original BalianLow Theorem, which we will call the Classical Balian-Low Theorem. The Classical BLT is much more difficult to prove than the Amalgam BLT. It was formulated independently by Balian [14] and by Low [149] for the case of orthonormal bases. A complete proof was given by Coifman, Daubechies, and Semmes [48], who also extended the Classical BLT to the case of Riesz bases. Battle [17] provided an elegant and entirely new proof based on the canonical commutation relations of quantum mechanics and thus demonstrated the intimate connection of the Classical BLT to the classical uncertainty principle. Battle's proof was adapted by Daubechies and Janssen [53] to provide another proof of the Classical BLT for Riesz bases (as well as obtaining further results for exact Gabor systems at the critical density). 
Theorem 7 (Classical Balian-Low Theorem). Choose $g \in L^{2}(\mathbf{R})$ and set $\alpha=\beta=1$. If $\mathcal{G}\left(g, \mathbf{Z}^{2}\right)$ is a Riesz basis for $L^{2}(\mathbf{R})$, then

$$
\left(\int_{\mathbf{R}}|t g(t)|^{2} d t\right)\left(\int_{\mathbf{R}}|\xi \hat{g}(\xi)|^{2} d \xi\right)=\infty
$$

It is shown in [23] that the Classical and Amalgam BLTs are independent, i.e., neither result implies the other. Further, Example 3.4 in [23] shows that the assumption that $\left(\int|t g(t)|^{2} d t\right)\left(\int|\xi \hat{g}(\xi)|^{2} d \xi\right)<\infty$ does not imply that $Z g$ is continuous.

Qualitatively, both versions of the BLT imply that if $\mathcal{G}\left(g, \mathbf{Z}^{2}\right)$ is a Riesz basis for $L^{2}(\mathbf{R})$, then either $g$ is not smooth or $g$ decays very slowly. By a change of variables, these theorems apply to all Gabor systems at the critical density $(\alpha \beta=1)$. Consequently, by the Density Theorem, "useful" Gabor frames are necessarily redundant $(\alpha \beta<1)$. For more details on Gabor systems at the critical density, see [129].

A BLT for Gabor frames on locally compact abelian groups was considered in [88]. An "approximate BLT" for $\alpha \beta$ close to 1 was obtained in [72, Theorem 3.6.13]. BalianLow Theorems with respect to other uncertainty principles are the subject of [20, 22, 21]. Gabardo and Han consider the BLT for frame and Riesz sequences and show its relation to the existence of a unique dual frame in [79]. Results for the BLT with respect to general lattices in higher dimensions appear in [67, 93, 19].

The Zak transform can also be used to study Gabor systems that are not at the critical density. The Zak transform is easiest to employ when $\alpha \beta=1 / N$, but Zibulski and Zeevi [193] have shown how the Zak transform can employed whenever $\alpha \beta$ is rational, cf. [26, 123].

\subsection{Wilson Bases}

Despite the fact that the Balian-Low Theorems imply that no "good" Gabor Riesz bases $\mathcal{G}(g, \alpha \mathbf{Z} \times \beta \mathbf{Z})$ exist, there does exist a remarkable construction known as Wilson bases which provide non-Gabor but still time-frequency-related Riesz or orthonormal bases for $L^{2}(\mathbf{R})$ that are generated by smooth windows. Wilson bases were first suggested by Wilson in [188]. The fact that they provide orthonormal bases for $L^{2}(\mathbf{R})$ was rigorously proved by Daubechies, Jaffard, and Journé [52], with further results by Auscher [2]. Feichtinger, Gröchenig, and Walnut proved that Wilson bases are unconditional bases for all of the modulation spaces [68] (see also [182]). We also mention that the local sine and cosine bases of Coifman and Meyer [45, 3] include many examples of Wilson and wavelet bases, and that the lapped transforms of Malvar [152] are closely related.

The construction of a Wilson basis starts with a "twice redundant" Gabor frame $\mathcal{G}\left(g, \frac{1}{2} \mathbf{Z} \times \mathbf{Z}\right)$ whose window satisfies the symmetry condition $g(t)=\overline{g(-t)}$, then forms linear combinations of elements, namely, $M_{n} T_{\frac{k}{2}} g \pm M_{-n} T_{\frac{k}{2}} g$, and finally "magically" extracts a subset which forms a Riesz basis for $L^{2}(\mathbf{R})$. Moreover, if the original Gabor frame was Parseval, then the resulting Wilson basis is orthonormal. Further, if the window $g$ has sufficient joint concentration in the time-frequency plane, namely $g \in M^{1}(\mathbf{R})$, then a Wilson basis will be an unconditional basis not only for $L^{2}(\mathbf{R})$, but simultaneously for all the modulation spaces $M^{p, q}$ (and there are corresponding analogues of these statements for the weighted modulation spaces), similar to Theorem 1 for the case of Gabor frames. We refer to the original literature, the exposition in [23, Section 4], and Sections 8.5 and 12.3 of [89] for more details. In higher dimensions, Wilson bases were constructed for Gabor systems on lattices of volume $\frac{1}{2}$ in [141], 


\subsection{The Walnut Representation}

We have already seen several fundamental principles in Gabor analysis, including the Wexler-Raz biorthogonality relations, the Duality Principle, and the Balian-Low Theorem. We briefly mention in this and the following section two additional fundamental principles. These are the Janssen Representation [122] and the Walnut Representation [181]. In particular, the Janssen Representation can be used to prove the Duality Principle and the Wexler-Raz relations.

If $\mathcal{G}(g, \alpha \mathbf{Z} \times \beta \mathbf{Z})$ is a Gabor frame for $L^{2}(\mathbf{R})$, recall that the Gabor frame operator is the positive, invertible operator $S$ on $L^{2}(\mathbf{R})$ defined by

$$
S f=\sum_{k, n \in \mathbf{Z}}\left\langle f, M_{\beta n} T_{\alpha k} g\right\rangle M_{\beta n} T_{\alpha k} g, \quad f \in L^{2}(\mathbf{R})
$$

The Walnut representation reformulates the frame operator solely in terms of translations of $g$ and $f$ [181]. In fact, we have the following more general result [89, Theorem 6.3.2].

Theorem 8 (Walnut Representation). If $g, \gamma \in W\left(L^{\infty}, \ell^{1}\right)$ and $\alpha, \beta>0$, then the following statements hold.

(a) The operator

$$
S_{g, \gamma} f=\sum_{k, n \in \mathbf{Z}}\left\langle f, M_{\beta n} T_{\alpha k} g\right\rangle M_{\beta n} T_{\alpha k} \gamma, \quad f \in L^{2}(\mathbf{R})
$$

is a bounded map of $L^{2}(\mathbf{R})$ into itself.

(b) The autocorrelation functions

$$
G_{n}=\sum_{k \in \mathbf{Z}} T_{\alpha k+\frac{n}{\beta}} \bar{g} \cdot T_{\alpha k} \gamma
$$

are bounded $\alpha$-periodic functions, and furthermore $\sum_{n \in \mathbf{Z}}\left\|G_{n}\right\|_{\infty}<\infty$.

(c) $S_{g, \gamma}$ can be written

$$
S_{g, \gamma} f=\frac{1}{\beta} \sum_{n \in \mathbf{Z}} G_{n} \cdot T_{\frac{n}{\beta}} f .
$$

A weak version of the Walnut representation is implicit in the proof of Theorems 2.6 and 2.7 of [48]. Specifically, assuming some complicated-looking hypotheses, Daubechies shows that (in our notation) $\sum_{k, n}\left|\left\langle f, M_{\beta n} T_{\alpha k} g\right\rangle\right|^{2}=\frac{1}{\beta} \sum_{n} \int G_{n}(t) f\left(t-\frac{n}{\beta}\right) \overline{f(t)} d t$; note that this is exactly $\left\langle S_{g, g} f, f\right\rangle$.

Walnut's improvements and extensive analysis appears in [181]. Some of those results are also included in the survey [113]. A detailed examination of the Walnut representation is made in [34].

In Theorem 1 we saw that if $g \in M^{1}(\mathbf{R})$ then Gabor frame expansions converge unconditionally in every modulation space $M^{p, q}(\mathbf{R})$. Walnut proved in [181] a different extension of Gabor frame expansions. If particular, he proved that if $g \in W\left(L^{\infty}, \ell^{1}\right)$ then the frame operator $S=S_{g, g}$ extends to a bounded mapping of $L^{p}(\mathbf{R})$ into itself for each $1 \leq p \leq \infty$, and also to a bounded mapping of $W\left(L^{\infty}, \ell^{1}\right)$ into itself (along with weighted versions of these results). This is interesting because of the fact that Walnut's proof for the 
case $p=2$ relies on the fact that the exponentials $\left\{\beta^{1 / 2} e^{2 \pi i \beta n t}\right\}_{n \in \mathbf{Z}}$ form an orthonormal basis for $L^{2}[0,1 / \beta]$. For $1<p<\infty, p \neq 2$, this family is still a Schauder basis for $L^{p}[0,1 / \beta]$, but it is a conditional basis, i.e., order of summation is important. Further, it is not a basis when $p=1$ or $p=\infty$. Extensions of Walnut's results were obtained in [95, 96], namely, it is shown there that if $g \in W\left(L^{\infty}, \ell^{1}\right)$, then Gabor frame expansions converge conditionally in every amalgam space $W\left(L^{p}, \ell^{q}\right)$ and that the Walnut representation (3.6) converges in the norm of $W\left(L^{p}, \ell^{q}\right)$ (along with weighted versions).

\subsection{The Janssen Representation}

Janssen's representation, which is also known as the Dual Lattice Representation, realizes the frame operator as a superposition of time-frequency shift operators along the adjoint lattice $\frac{1}{\beta} \mathbf{Z} \times \frac{1}{\alpha} \mathbf{Z}$. A weak form was proved by Tolimieri and Orr [179], and the stronger forms were independently introduced in [122] and in [54] (where it is called the WexlerRaz identity). We refer to [67] or [89, Section 7.2] for additional details on the Janssen representation, and to [127] for a survey on Gabor frame operator representations.

Theorem 9 (Janssen Representation). Let $\alpha, \beta>0$ be given, and assume that $g$, $\gamma \in L^{2}(\mathbf{R})$ satisfy Condition A', i.e.,

$$
\sum_{k, n \in \mathbf{Z}}\left|\left\langle\gamma, M_{\frac{n}{\beta}} T_{\frac{k}{\alpha}} g\right\rangle\right|<\infty
$$

Then the operator $S_{g, \gamma}$ defined in (3.5) can be written

$$
S_{g, \gamma}=\frac{1}{\alpha \beta} \sum_{k, n \in \mathbf{Z}}\left\langle\gamma, M_{\frac{n}{\alpha}} T_{\frac{k}{\beta}} g\right\rangle M_{\frac{n}{\alpha}} T_{\frac{k}{\beta}}
$$

with convergence of this series in operator norm.

In particular, if $\mathcal{G}\left(g, \alpha \mathbf{Z}^{d} \times \beta \mathbf{Z}^{d}\right)$ is a frame and $\mathcal{G}\left(\tilde{g}, \alpha \mathbf{Z}^{d} \times \beta \mathbf{Z}^{d}\right)$ is its canonical dual frame, then $S_{g, \tilde{g}}=I$, the identity operator. As a consequence,

$$
\frac{1}{\alpha \beta}\left\langle\tilde{g}, M_{\frac{n}{\alpha}} T_{\frac{k}{\beta}} g\right\rangle=\delta_{n 0} \delta_{k 0} .
$$

This (correctly) suggests that the Janssen representation is a fundamental idea underlying the Wexler-Raz biorthogonality relations and the Duality Principle, see [122, p. 408].

Condition A' (which for the case $g=\gamma$ is called Condition A) was introduced by Tolimieri and Orr in [179]. It is a technical condition whose validity is difficult to verify in general. On the other hand, it is satisfied for all $\alpha, \beta$ if $g, \gamma \in M^{1}$. Condition A' and membership in $W\left(L^{\infty}, \ell^{1}\right)$ are distinct requirements, but both are satisfied for all functions $g, \gamma \in M^{1}$. As Gröchenig remarks [89, p. 256], Condition $\mathrm{A}^{\prime}$ and the amalgam space $W\left(L^{\infty}, \ell^{1}\right)$ each provide suitable window classes for $L^{2}$-theory, but for wider applicability and robustness the appropriate window class is $M^{1}$ (and its weighted counterparts).

To give one brief intuition for the Janssen representation, recall that the frame operator $S$ defined in (3.4) commutes with the lattice shifts $T_{\alpha}$ and $M_{\beta}$. Hence $S$ commutes with all operators in the von Neumann algebra $\mathcal{M}_{\alpha, \beta}$ generated by $T_{\alpha}$ and $M_{\beta}$, i.e., $S$ belongs to the commutant $\mathcal{M}_{\alpha, \beta}^{\prime}$ of $\mathcal{M}_{\alpha, \beta}$ (we refer to [178] for a general reference on von Neumann algebras). However, $T_{\frac{1}{\beta}}$ and $M_{\frac{1}{\alpha}}$ also commute with $T_{\alpha}$ and $M_{\beta}$, i.e., $T_{\frac{1}{\beta}}, M_{\frac{1}{\alpha}} \in \mathcal{M}_{\alpha, \beta}^{\prime}$, so 
we have the inclusion $\mathcal{M}_{\frac{1}{\beta}, \frac{1}{\alpha}} \subset \mathcal{M}_{\alpha, \beta}^{\prime}$. In fact, equality holds. According to Folland [74], this fact is a special case of a theorem of Takesaki, see [177, Theorem 4]. One proof of this equality appears in [54, App. 6.1]. In any case, it follows that $S \in \mathcal{M}_{\frac{1}{\beta}, \frac{1}{\alpha}}=\mathcal{M}_{\alpha, \beta}^{\prime}$, and hence should be representable in terms of the operators $T_{\frac{k}{\beta}}$ and $M_{\frac{n}{\alpha}}$. The specific form of this representation is Theorem 9 .

\section{Lattice Gabor Systems}

All of the results mentioned so far for rectangular lattice Gabor systems in dimension $d=1$ can be generalized to rectangular lattice Gabor systems $\mathcal{G}\left(g, \alpha \mathbf{Z}^{d} \times \beta \mathbf{Z}^{d}\right)$ in arbitrary dimensions.

However, as we shall see in the following sections, care must be taken when considering other lattices, even for the seemingly simple case of more general rectangular lattices such as

$$
\Lambda=\prod_{i=1}^{d} \alpha_{i} \mathbf{Z} \times \prod_{i=1}^{d} \beta_{i} \mathbf{Z} .
$$

For example, let $\varphi(t)=2^{1 / 4} e^{-\pi t^{2}}$ be the 1-dimensional Gaussian, and let

$$
\Phi(t)=2^{d / 4} e^{-\pi t \cdot t}=\prod_{i=1}^{d} \varphi\left(t_{i}\right)
$$

be the $d$-dimensional Gaussian. Consider $\Phi$ with respect to a lattice $\Lambda$ of the form given in (4.1). Since $\Phi$ is a tensor product of 1-dimensional Gaussians, a first expectation may be that the existence result for the 1-dimensional Gaussian generalizes to say that $\mathcal{G}(\Phi, \Lambda)$ is a frame whenever the volume $\operatorname{vol}(\Lambda)=\alpha_{1} \cdots \alpha_{d} \cdot \beta_{1} \cdots \beta_{d}$ of the lattice satisfies $0<\operatorname{vol}(\Lambda) \leq 1$. However, this is false.

In fact, because $\Phi$ and $\Lambda$ are separable (tensor products), $\mathcal{G}(\Phi, \Lambda)$ is complete in $L^{2}\left(\mathbf{R}^{d}\right)$ if and only if $\mathcal{G}\left(\varphi, \alpha_{i} \mathbf{Z} \times \beta_{i} \mathbf{Z}\right)$ is complete in $L^{2}(\mathbf{R})$ for $i=1, \ldots, d$. However, we know that this requires $\alpha_{i} \beta_{i} \leq 1$ for each $i$. Hence if $0<\operatorname{vol}(\Lambda)<1$ but $\alpha_{i} \beta_{i}>1$ for some $i$, then $\mathcal{G}(\Phi, \Lambda)$ will be incomplete. Thus we can create lattices with arbitrarily small volumes such that $\mathcal{G}(\Phi, \Lambda)$ is incomplete.

Despite these facts for the Gaussian window, the extension of the Density Theorem to general windows and arbitrary lattices in higher dimensions is formulated in terms of the volume of the lattice. In particular, even for a lattice $\Lambda$ of the form given in (4.1), we will see that there exists some $g \in L^{2}\left(\mathbf{R}^{d}\right)$ such that $\mathcal{G}(g, \Lambda)$ is a frame if and only if $0<\operatorname{vol}(\Lambda) \leq 1$.

For a general lattice $A\left(\mathbf{Z}^{2 d}\right)$ in higher dimensions, its volume is defined to be

$$
\operatorname{vol}\left(A\left(\mathbf{Z}^{2 d}\right)\right)=|\operatorname{det}(A)|
$$

Thus, the volume of a lattice is the area of a fundamental domain for the lattice. It is this quantity that will distinguish between the various cases in the Density Theorem.

Before discussing the extension of the Density Theorem to general lattice Gabor systems in higher dimensions, we begin with the special case of symplectic lattices. 


\subsection{Symplectic Lattices}

The natural first setting to look for an extension of the Density Theorem to higher dimensions would seem to be the special case of Gabor systems $\mathcal{G}(g, \Lambda)$ where $\Lambda$ is a symplectic lattice. Symplectic lattices are closely related to metaplectic transforms and the representation theory of the Heisenberg group, and we refer to [89, Section 9.4] for a detailed exposition; see also the short discussion in [102].

A symplectic lattice is a lattice of the form

$$
\Lambda=\alpha A\left(\mathbf{Z}^{2 d}\right),
$$

where $A$ is a symplectic matrix and $\alpha>0$. Every symplectic matrix satisfies $\operatorname{det}(A)=1$ and hence a symplectic lattice $\Lambda=\alpha A\left(\mathbf{Z}^{2 d}\right)$ has volume $\alpha^{2 d}$. For $d=1$, every lattice is symplectic, but this is not the case when $d>1$.

The precise definition of a symplectic lattice is given below, but more important for us than the actual definition is the following fact, cf. [89, Proposition 9.4.4]. Here $\mathbf{T}=\{z \in \mathbf{C}:|z|=1\}$ is the unit circle in the complex plane.

Proposition 4. Let $\Lambda=\alpha A\left(\mathbf{Z}^{2 d}\right)$ be a symplectic lattice. Then there exists a unitary operator $\mu_{A}: L^{2}\left(\mathbf{R}^{d}\right) \rightarrow L^{2}\left(\mathbf{R}^{d}\right)$ and a function $c_{A}: \alpha \mathbf{Z}^{2 d} \rightarrow \mathbf{T}$ such that

$$
\mathcal{G}(g, \Lambda)=\left\{c_{A}(\alpha k, \alpha n) \mu_{A}\left(M_{\alpha n} T_{\alpha k} h\right)\right\}_{k, n \in \mathbf{Z}^{d}}
$$

where $h=\mu_{A}^{-1}(g)$.

The operator $\mu_{A}$ is called a metaplectic transform.

If the scalars $c_{A}(\alpha k, \alpha n)$ were to be removed, then the rightmost set in (4.2) would be $\mu_{A}\left(\mathcal{G}\left(h, \alpha \mathbf{Z}^{2 d}\right)\right)$, which is the image of a rectangular lattice Gabor system under a unitary map. Yet although the right-hand set in (4.2) is not precisely a Gabor system, since the property of being complete, a frame, a Riesz basis, or a Riesz sequence is preserved both by unitary mappings and by multiplication of the elements by scalars of unit modulus, we obtain the following.

Proposition 5. Let $\Lambda=\alpha A\left(\mathbf{Z}^{2 d}\right)$ be a symplectic lattice, and let $g \in L^{2}\left(\mathbf{R}^{d}\right)$. Then $\mathcal{G}(g, \Lambda)$ is complete, a frame, a Riesz basis, or a Riesz sequence in $L^{2}\left(\mathbf{R}^{d}\right)$ if and only if the same is true of $\mathcal{G}\left(h, \alpha \mathbf{Z}^{2 d}\right)$, where $h=\mu_{A}^{-1}(g)$.

Since $\operatorname{det}(A)=1$, the volume of the rectangular lattice $\alpha \mathbf{Z}^{2 d}$ and the symplectic lattice $\alpha A\left(\mathbf{Z}^{2 d}\right)$ are equal. Hence the Density Theorem immediately extends from rectangular lattices to all symplectic lattices, with the volume of the symplectic lattice being the quantity that distinguishes between the various cases in the Density Theorem. Additionally, we also immediately conclude that for each symplectic lattice $\Lambda$ with $0<\operatorname{vol}(\Lambda) \leq 1$, there exists a $g \in L^{2}\left(\mathbf{R}^{d}\right)$ such that $\mathcal{G}(g, \Lambda)$ is a frame for $L^{2}\left(\mathbf{R}^{d}\right)$.

Example 1. Consider $d=1$ and let $A$ be the shear matrix $A=\left[\begin{array}{ll}1 & 0 \\ r & 1\end{array}\right]$. Set

$$
\Lambda=\alpha A\left(\mathbf{Z}^{2}\right)=\{(\alpha k, \alpha n+r \alpha k)\}_{k, n \in \mathbf{Z}}
$$

Let $\mu_{A}: L^{2}(\mathbf{R}) \rightarrow L^{2}(\mathbf{R})$ be multiplication by the "chirp" $e^{\pi i r t^{2}}$, i.e.,

$$
\mu_{A} f(t)=e^{\pi i r t^{2}} f(t) .
$$


Then $\mu_{A}$ is a unitary mapping of $L^{2}(\mathbf{R})$ onto itself. Given $g \in L^{2}(\mathbf{R})$, define $h(t)=$ $\mu_{A}^{-1} g(t)=e^{-\pi i r t^{2}} g(t)$. A direct calculation shows that

$$
M_{\alpha n+\alpha r k} T_{\alpha k} g=e^{2 \pi i r(\alpha k)^{2}} \mu_{A}\left(M_{\alpha n} T_{\alpha k} h\right) .
$$

Hence, if we define $c_{A}(x, \xi)=e^{\pi i r x^{2}}$, then

$$
\mathcal{G}\left(g, \alpha A\left(\mathbf{Z}^{2}\right)\right)=\left\{M_{\alpha n+r \alpha k} T_{\alpha k} g\right\}_{k, n \in \mathbf{Z}}=\left\{c_{A}(\alpha k, \beta n) \mu_{A}\left(M_{\alpha n} T_{\alpha k} h\right)\right\}_{k, n \in \mathbf{Z}} .
$$

Therefore, we can see directly for this case that $\mathcal{G}\left(g, \alpha A\left(\mathbf{Z}^{2}\right)\right)$ is complete, a frame, a Riesz basis, or a Riesz sequence if and only if the same is true of of $\mathcal{G}\left(h, \alpha \mathbf{Z}^{2}\right)$.

Remark 1. If we were to define a Gabor system using time-frequency shifts better matched to the structure of the Heisenberg group, specifically,

$$
G(g, \Lambda)=\{\rho(x, \xi) g\}_{(x, \xi) \in \Lambda}, \quad \text { where } \rho(x, \xi)=e^{\pi i x \cdot \xi} T_{x} M_{\xi},
$$

then the constants $c_{A}(\alpha k, \alpha n)$ in (4.2) all become identically 1. Hence, with this definition, if $\Lambda=\alpha A\left(\mathbf{Z}^{2 d}\right)$ is a symplectic lattice then

$$
G(g, \Lambda)=G\left(g, \alpha A\left(\mathbf{Z}^{2 d}\right)\right)=\mu_{A}\left(G\left(h, \alpha \mathbf{Z}^{2 d}\right)\right)
$$

is the image of a rectangular lattice Gabor system under the metaplectic transform $\mu_{A}$.

The precise definition of a symplectic lattice is as follows.

Definition 3 (Symplectic Lattices).

(a) The symplectic form on $\mathbf{R}^{2 d}$ is

$$
\left[z, z^{\prime}\right]=x^{\prime} \cdot \xi-x \cdot \xi^{\prime}, \quad z=(x, \xi), z^{\prime}=\left(x^{\prime}, \xi^{\prime}\right) \in \mathbf{R}^{2 d} .
$$

(b) A $2 d \times 2 d$ invertible matrix $A$ is a symplectic matrix if it preserves the symplectic form on $\mathbf{R}^{2 d}$, i.e., if $\left[A z, A z^{\prime}\right]=\left[z, z^{\prime}\right]$ for $z, z^{\prime} \in \mathbf{R}^{2 d}$. The symplectic group $\operatorname{Sp}(d)$ is the group of all $2 d \times 2 d$ symplectic matrices.

(c) We say that a lattice $\Lambda$ is a symplectic lattice if $\Lambda=\alpha A\left(\mathbf{Z}^{2 d}\right)$ where $A$ is a symplectic matrix and $\alpha>0$.

If we write a $2 d \times 2 d$ matrix in block form as $M=\left[\begin{array}{cc}A & B \\ C & D\end{array}\right]$, then $M$ is symplectic if and only if $A C^{*}=A^{*} C, B D^{*}=B^{*} D$, and $A^{*} D-C^{*} B=I$ [89, Lemma 9.4.1]. In particular, if $M=\left[\begin{array}{rr}A & 0 \\ 0 & D\end{array}\right]$ is block diagonal, then $M$ is symplectic if and only if $A^{*} D=I$. Thus, a lattice with the rectangular form given in (4.1) is symplectic if and only if the matrix $\operatorname{diag}\left(\alpha_{1}, \ldots, \alpha_{d}, \beta_{1}, \ldots, \beta_{d}\right)$ is a multiple of a symplectic matrix, which requires that all the products $\alpha_{i} \beta_{i}$ be equal. Consequently, all rectangular lattices $\alpha \mathbf{Z}^{d} \times \beta \mathbf{Z}^{d}$ are symplectic, which is one reason they are so convenient for Gabor analysis in higher dimensions.

\subsection{General Lattices}

As we have seen, the extension of the Density Theorem to symplectic lattices is immediate. Many other results in Gabor analysis also extend easily to symplectic lattices, but often it is 
unknown if extensions to general lattices exist. For example, it is known that the Classical Balian-Low Theorem for the special case of orthonormal bases extends to all lattices. Yet for general Riesz bases, the Classical BLT is only known to hold in full generality for symplectic lattices $[93,19]$. A weak version of the Classical BLT is known to hold for nonsymplectic lattices, but currently it is not known if any version of the Amalgam BLT extends to non-rectangular lattices, symplectic or otherwise.

Therefore, it is quite interesting that the Density Theorem does extend to completely arbitrary lattices, and furthermore that for each lattice with volume at most 1 , there exists a Gabor frame corresponding to that lattice.

Theorem 10 (Density Theorem for Lattices). Let $g \in L^{2}\left(\mathbf{R}^{d}\right)$ and let $\Lambda=A\left(\mathbf{Z}^{2 d}\right)$ where $A$ is an invertible $2 d \times 2 d$ matrix. Then the following statements hold.

(a) If $\operatorname{vol}(A)>1$, then $\mathcal{G}\left(g, A\left(\mathbf{Z}^{2 d}\right)\right)$ is incomplete in $L^{2}\left(\mathbf{R}^{d}\right)$.

(b) If $\mathcal{G}\left(g, A\left(\mathbf{Z}^{2 d}\right)\right)$ is a frame for $L^{2}\left(\mathbf{R}^{d}\right)$, then $0<\operatorname{vol}(A) \leq 1$.

(c) $\mathcal{G}\left(g, A\left(\mathbf{Z}^{2 d}\right)\right)$ is a Riesz basis for $L^{2}\left(\mathbf{R}^{d}\right)$ if and only if it is a frame for $L^{2}\left(\mathbf{R}^{d}\right)$ and $\operatorname{vol}(A)=1$.

(d) If $\mathcal{G}\left(g, A\left(\mathbf{Z}^{2 d}\right)\right)$ is a Riesz sequence in $L^{2}\left(\mathbf{R}^{d}\right)$, then $\operatorname{vol}(A) \geq 1$.

Parts (b) and (c) of the Density Theorem are immediate consequences of Ramanathan and Steger's results on the Density Theorem for irregular Gabor frames in [158] (see Section 5 below). More precisely, [158] applies to $d=1$; the extension to higher dimensions was made in [42]. For the case of separable lattices, part (b) was also obtained in [162].

Parts (b), (c), and (d) are consequences of the extension by Feichtinger and Kozek of the Wexler-Raz relations and the Duality Principle to arbitrary lattices [71], which we discuss in more detail below.

For the case of separable lattices, part (a) was proved by Han and Wang in [101], and a different proof was given by Gabardo and Han in [78]. However, it appears that part (a) of the Density Theorem was only established in its full generality recently, by Bekka [18]. Moreover, Bekka provided a positive answer to the existence question for arbitrary lattices. We discuss this in more detail next.

\subsection{Existence for General Lattices}

As noted at the beginning of Section 4 , there exist lattices $\Lambda$ with arbitrarily small volume such that the Gabor system $\mathcal{G}(\Phi, \Lambda)$ generated by the Gaussian function $\Phi$ is incomplete. If $\Lambda$ is symplectic, say $\Lambda=\alpha A\left(\mathbf{Z}^{2 d}\right)$, then, as described above, we can take any rectangular lattice Gabor frame $\mathcal{G}\left(g, \alpha \mathbf{Z}^{2 d}\right)$ (which is easy to construct via the "Painless Nonorthogonal Expansions" approach), and use a metaplectic transform to obtain an $h$ such that $\mathcal{G}(h, \Lambda)$ will be a frame. However, if $\Lambda$ is not symplectic, then it is not at all obvious whether there will exist some $g$ such that $\mathcal{G}(g, \Lambda)$ is a frame.

Thus, it is quite interesting that Bekka proved in [18] that for any lattice $\Lambda$ with $0<\operatorname{vol}(\Lambda) \leq 1$ there will exist some window $g$ such that $\mathcal{G}(g, \Lambda)$ will be a frame. Specifically, Bekka proved the following, cf. [18, Theorem 4]; note that this is only a special case of the more general results that are derived in that article. ${ }^{4}$

\footnotetext{
${ }^{4}$ Bekka himself attributes this result to Feichtinger and Kozek [71], but while that article does contain
} many results for Gabor systems on arbitrary lattices, it does not contain Theorem 11. 
Theorem 11 (Existence of Lattice Gabor Frames). Let $\Lambda=A\left(\mathbf{Z}^{2 d}\right)$ be a lattice in $\mathbf{R}^{2 d}$. Then the following statements are equivalent.

(a) $\operatorname{vol}(\Lambda) \leq 1$.

(b) There exists a $g \in L^{2}\left(\mathbf{R}^{d}\right)$ such that $\mathcal{G}(g, \Lambda)$ is complete in $L^{2}\left(\mathbf{R}^{d}\right)$.

(c) There exists a $g \in L^{2}\left(\mathbf{R}^{d}\right)$ such that $\mathcal{G}(g, \Lambda)$ is a frame for $L^{2}\left(\mathbf{R}^{d}\right)$.

The following statements are also equivalent.

(a') $\operatorname{vol}(\Lambda)=1$.

(b') There exists a $g \in L^{2}\left(\mathbf{R}^{d}\right)$ such that $\mathcal{G}(g, \Lambda)$ is a Riesz basis for $L^{2}\left(\mathbf{R}^{d}\right)$.

(c') There exists a $g \in L^{2}\left(\mathbf{R}^{d}\right)$ such that $\mathcal{G}(g, \Lambda)$ is an orthonormal basis for $L^{2}\left(\mathbf{R}^{d}\right)$.

For the case of separable lattices, i.e., $\Lambda=A\left(\mathbf{Z}^{d}\right) \times B\left(\mathbf{Z}^{d}\right)$, the equivalences in Theorem 11 were earlier proved in $[101,78]$ (see also [102]).

Unfortunately, Bekka's proof of Theorem 11 is not constructive. On the other hand, the proof in [101] of Theorem 11 for the special case of separable lattices $\Lambda=A\left(\mathbf{Z}^{d}\right) \times B\left(\mathbf{Z}^{d}\right)$ is constructive. The window constructed in [101] is the characteristic function of a set. In some cases this set will be compact; in particular, this is the case if $A, B$ have all rational entries. However, in general, even for separable lattices it is not currently known if there exists a compactly supported $g \in L^{2}\left(\mathbf{R}^{d}\right)$ such that $\mathcal{G}(g, \Lambda)$ is a frame.

\subsection{More on Arbitrary Lattices}

As we have mentioned, many results for rectangular lattice Gabor systems generalize naturally to the setting of symplectic lattices. Surprisingly, Feichtinger and Kozek proved in [71] that a number of fundamental results for rectangular lattice Gabor systems actually extend to arbitrary lattice Gabor systems in higher dimensions (see also [67] for the case $d=1$ ). These include the Janssen representation, the Wexler-Raz biorthogonality relations, and the Duality Principle. On the other hand, the question of whether other basic results, such as the Balian-Low Theorems, extend to arbitrary lattices remains open.

A fundamental notion introduced in [71] is the concept of the adjoint lattice. Feichtinger and Kozek's setting was more general than considered in this article; instead of the domain $\mathbf{R}^{d}$, they considered Gabor systems whose domain was an elementary locally compact abelian group (see [71] for the full definition). For lattices in $\mathbf{R}^{2 d}$, the adjoint is defined as follows.

Definition 4 (Adjoint Lattice). Let $\Lambda=A\left(\mathbf{Z}^{2 d}\right)$ be a lattice. For $\mu=(x, \xi) \in \mathbf{R}^{2 d}$, define $\pi(\mu)=M_{\xi} T_{x}$. Then the adjoint lattice of $\Lambda$ is

$$
\Lambda^{\circ}=\left\{\mu \in \mathbf{R}^{2 d}: \pi(\lambda) \pi(\mu)=\pi(\mu) \pi(\lambda) \text { for all } \lambda \in \Lambda\right\} .
$$

Remark 2. (a) The adjoint lattice should not be confused with the dual lattice

$$
\Lambda^{\perp}=\left\{\mu \in \mathbf{R}^{2 d}: e^{2 \pi i \lambda \cdot \mu}=1 \text { for all } \lambda \in \Lambda\right\}=\left(A^{\mathrm{T}}\right)^{-1}\left(\mathbf{Z}^{2 d}\right),
$$

which plays a role in many formulas in Fourier analysis, such as the Poisson Summation Formula. The dual lattice is the annihilator of $\Lambda$ with respect to the Fourier transform. Feichtinger and Kozek prove in [71] that the adjoint lattice is the annihilator of $\Lambda$ with 
respect to the symplectic Fourier transform. Additional discussion of the adjoint lattice and the Wexler-Raz relations can be found in [72]. For example, it is noted there that

$$
\Lambda^{\circ}=\left(\begin{array}{cc}
0 & I \\
-I & 0
\end{array}\right)\left(A^{\mathrm{T}}\right)^{-1}\left(\mathbf{Z}^{2 d}\right)
$$

(b) For the case of a rectangular lattice $\Lambda=\alpha \mathbf{Z}^{d} \times \beta \mathbf{Z}^{d}$, the adjoint and dual lattices are

$$
\Lambda^{\circ}=\frac{1}{\beta} \mathbf{Z}^{d} \times \frac{1}{\alpha} \mathbf{Z}^{d}, \quad \Lambda^{\perp}=\frac{1}{\alpha} \mathbf{Z}^{d} \times \frac{1}{\beta} \mathbf{Z}^{d} .
$$

(c) If $\Lambda$ is a symplectic lattice, then the adjoint lattice is simply $\Lambda^{\circ}=\operatorname{vol}(\Lambda)^{-1 / d} \Lambda$.

(d) Ron and Shen's formulation of the Duality Principle in [162] was actually for lattice Gabor systems $\mathcal{G}(g, \Lambda)$ where $\Lambda$ is separable, i.e., $\Lambda=A\left(\mathbf{Z}^{d}\right) \times B\left(\mathbf{Z}^{d}\right)$ with $d \times d$ invertible matrices $A, B$. Moreover, they introduced the notion of an adjoint system of $\mathcal{G}(g, \Lambda)$, which in our notation is the Gabor system $\mathcal{G}(g, \Gamma)$ with $\Gamma=B\left(\mathbf{Z}^{d}\right)^{\perp} \times A\left(\mathbf{Z}^{d}\right)^{\perp}$. Since for a separable lattice we have

$$
\left(A\left(\mathbf{Z}^{d}\right) \times B\left(\mathbf{Z}^{d}\right)\right)^{\circ}=B\left(\mathbf{Z}^{d}\right)^{\perp} \times A\left(\mathbf{Z}^{d}\right)^{\perp},
$$

we see that Ron and Shen had the notion of the adjoint lattice for the case of separable lattices. However, the idea of defining the adjoint of an arbitrary lattice by commutation conditions is due to Feichtinger and Kozek.

We close this section by quoting Gröchenig [89, p. 201]:

At this time the difference between symplectic lattices and general timefrequency lattices is not yet fully understood. While the structure results for Gabor frames hold for arbitrary lattices, many other results, such as a symbolic calculus for frame operators, seem to break down for nonsymplectic lattices.

\section{Irregular Gabor Systems}

Gabor systems $\mathcal{G}(g, \Lambda)$ where $\Lambda$ has no or very limited structure arise very naturally. For example, it follows from the coorbit theory of Feichtinger and Gröchenig [64, 65, 86] that if $g$ lies in the modulation space $M^{1}$, then $\mathcal{G}(g, \Lambda)$ will be a frame for every "sufficiently dense" set of indices $\Lambda$. Liu and Wang [148] have shown that there exist Gabor systems $\mathcal{G}(g, \Lambda)$ with aperiodic $\Lambda$ that are even orthonormal bases for $L^{2}\left(\mathbf{R}^{d}\right)$; this is related to tilings in $\mathbf{R}^{d}$ by cubes. Lyubarskii [150] and Seip/Wallstén [164, 168] have, for the case of the one-dimensional Gaussian window $\varphi(t)=2^{1 / 4} e^{-\pi t^{2}}$, completely characterized all $\Lambda$ such that $\mathcal{G}(\varphi, \Lambda)$ is a frame for $L^{2}(\mathbf{R})$.

Irregular Gabor systems also arise naturally from perturbations of lattice Gabor systems, e.g., due to jitter or noise. Suppose that $\mathcal{G}\left(g, A\left(\mathbf{Z}^{2 d}\right)\right)$ is a lattice Gabor frame and that the elements of this lattice are slightly perturbed. Under appropriate hypotheses on $g$ and $\Lambda$, local perturbation theorems, such as those in [180, 59,44,40,173], may be applied to derive conditions under which the resulting system $\mathcal{G}(g, \Lambda)$ will still be a frame.

However, such local perturbation results do not allow us to prove general theorems on the properties of Gabor systems with irregular index sets. Further, all of the tools and techniques that have be discussed so far in this article are inadequate for the study of irregular 
Gabor systems. For example, if we take a lattice $A\left(\mathbf{Z}^{2 d}\right)$ and perturb even one single point of the lattice, then the resulting set $\Lambda$ is no longer a lattice. Every "algebraic" tool that has so far been discussed, including the Walnut representation, the Janssen representation, the Duality Principle, the Wexler-Raz biorthogonality relations, the Zak transform, and von Neumann algebra techniques, is rendered inapplicable to the study of $\mathcal{G}(g, \Lambda)$.

For the irregular setting new tools are needed, and these were first supplied by H. Landau [145], Ramanathan and Steger [158], and Janssen [126], who provided the first extensions of the Density Theorem to irregular Gabor frames (and, in the case of [126], to more general systems).

Yet there are surprises. In particular, unlike the lattice case, for irregular Gabor systems there is a distinction in the Density Theorem between the cases of complete Gabor systems and Gabor systems that are frames. The Density Theorem does apply to frames; specifically, it says that if $\mathcal{G}(g, \Lambda)$ is a frame then $\Lambda$ cannot be "too sparse," in the sense that the lower Beurling density of $\Lambda$ must satisfy $D^{-}(\Lambda) \geq 1$. However, the Density Theorem does not apply to general complete sequences: There exist $g$ and $\Lambda$ with arbitrarily small or even zero Beurling density such that $\mathcal{G}(g, \Lambda)$ is complete (but not a frame) in $L^{2}\left(\mathbf{R}^{d}\right)$.

In general, irregular Gabor systems are considerably more intractable than lattice Gabor systems, and few results have been available until quite recently. One fundamental problem is that, unlike the lattice case, the canonical dual frame of an irregular Gabor frame $\mathcal{G}(g, \Lambda)$ need not itself be a Gabor frame. In the rectangular lattice case, it is easy to see that the time-frequency shifts $M_{\beta n} T_{\alpha k}$ commute with the frame operator, but in the irregular setting the time-frequency shifts $M_{\xi} T_{x}$ with $(x, \xi) \in \Lambda$ need no longer commute with the frame operator. Consequently, while $\mathcal{G}(g, \Lambda)=\left\{M_{\xi} T_{x} g\right\}_{(x, \xi) \in \Lambda}$ is a set of timefrequency shifts of $g$, we cannot say that the dual frame is a set of time-frequency shifts of some single window $\tilde{g}$. Instead, we can only say that the canonical dual is some sequence $\tilde{\mathcal{G}}=\left\{\tilde{g}_{x, \xi}\right\}_{(x, \xi) \in \Lambda}$ of functions in $L^{2}\left(\mathbf{R}^{d}\right)$. We will address this problem in more detail in Section 6.3 below.

In addition to those article mentioned above, some article dealing with irregular Gabor frames include $[86,87,42,55,43,101,174,11,148,175,138]$. The concept of localized frames, discussed in Section 6 below, has provided a new viewpoint for understanding irregular Gabor systems.

\subsection{Beurling Density}

In Theorems 3 and 10, the value that distinguishes between the various cases is the volume of the lattice, which is the area of a fundamental domain for the lattice. In the irregular setting there is no analogue of a fundamental domain, and instead it is the Beurling density of $\Lambda$ that distinguishes between the various cases. Beurling density measures in some sense the average number of points inside unit cubes. However, because the points are not uniformly distributed, there is not a single definition, but rather we obtain lower and upper limits to the average density. More precisely, we count the average number of points inside cubes of larger and larger radii and take the limit. The precise definition and some basic facts about Beurling density are as follows, cf. [42].

Definition/Facts 4. Let $Q_{r}(z)$ denote the cube in $\mathbf{R}^{2 d}$ centered at $z \in \mathbf{R}^{2 d}$ with side lengths $r$, and let $|E|$ denote the cardinality of a set $E$. Let $\Lambda$ be a sequence of points in $\mathbf{R}^{2 d}$. 
(a) The lower and upper Beurling densities of $\Lambda$ are, respectively,

$$
D^{-}(\Lambda)=\liminf _{r \rightarrow \infty} \inf _{z \in \mathbf{R}^{2 d}} \frac{\left|\Lambda \cap Q_{r}(z)\right|}{r^{2 d}} \text { and } D^{+}(\Lambda)=\limsup _{r \rightarrow \infty} \sup _{z \in \mathbf{R}^{2 d}} \frac{\left|\Lambda \cap Q_{r}(z)\right|}{r^{2 d}} .
$$

(b) The definition of Beurling density is unchanged if instead of dilating cubes we dilate any compact set with unit measure whose boundary has measure zero [144]; see also the new characterizations of Beurling density in [138].

(c) The Beurling density of a rectangular lattice is

$$
D^{-}\left(\alpha \mathbf{Z}^{d} \times \beta \mathbf{Z}^{d}\right)=D^{+}\left(\alpha \mathbf{Z}^{d} \times \beta \mathbf{Z}^{d}\right)=\frac{1}{(\alpha \beta)^{d}},
$$

and the density of a general lattice $A\left(\mathbf{Z}^{2 d}\right)$ is

$$
D^{-}\left(A\left(\mathbf{Z}^{2 d}\right)\right)=D^{+}\left(A\left(\mathbf{Z}^{2 d}\right)\right)=\frac{1}{|\operatorname{det}(A)|}=\frac{1}{\operatorname{vol}(A)} .
$$

(d) We say that $\Lambda$ is uniformly separated if $\inf \{|\lambda-\mu|: \lambda \neq \mu \in \Lambda\}>0$. If $\Lambda$ is uniformly separated, then $D^{+}(\Lambda)<\infty$, but the converse is false; for example, $D^{+}\left(\mathbf{Z}^{2} \cup \sqrt{2} \mathbf{Z}^{2}\right)<\infty$.

(e) $D^{+}(\Lambda)<\infty$ if and only if $\Lambda$ is relatively uniformly separated, which means that we can write $\Lambda$ as the union of finitely many subsequences $\Lambda_{1}, \ldots, \Lambda_{N}$, each of which is uniformly separated.

(f) $\quad D^{-}(\Lambda)>0$ if and only if there exists an $R>0$ such that every cube $Q_{R}(z)$ with $z \in \mathbf{R}^{2 d}$ contains at least one element of $\Lambda$.

It was shown in [42, Theorem 3.1] that if $g \in L^{2}\left(\mathbf{R}^{d}\right)$ and $\Lambda$ is a sequence in $\mathbf{R}^{2 d}$ such that $\mathcal{G}(g, \Lambda)$ is a Bessel sequence, then necessarily $D^{+}(\Lambda)<\infty$.

Conversely, we have the following important fact, that if a window $g$ lies in the Feichtinger algebra $M^{1}\left(\mathbf{R}^{d}\right)$ then $\mathcal{G}(g, \Lambda)$ is Bessel for every finite density sequence $\Lambda$. This is a consequence of Theorem 2, which states that if $g \in M^{1}\left(\mathbf{R}^{d}\right)$ and $f$ is any function in $L^{2}\left(\mathbf{R}^{d}\right)$, then the STFT $V_{g} f$ is well-behaved, namely, $V_{g} f$ belongs to the amalgam space $W\left(\mathcal{C}, \ell^{2}\right)$.

Theorem 12. If $g \in M^{1}\left(\mathbf{R}^{d}\right)$ and $\Lambda$ is any sequence in $\mathbf{R}^{2 d}$ such that $D^{+}(\Lambda)<\infty$, then $\mathcal{G}(g, \Lambda)$ is a Bessel sequence.

Proof. Let $Q=[0,1]^{2 d}$. Since $D^{+}(\Lambda)<\infty$, there is a finite number $K$ such that any translate $Q+j$ of $Q$ can contain at most $K$ points of $\Lambda$. If $f \in L^{2}\left(\mathbf{R}^{d}\right)$, then we have $V_{g} f \in W\left(\mathcal{C}, \ell^{2}\right)\left(\mathbf{R}^{2 d}\right)$ by Proposition 2 , and hence

$$
\begin{aligned}
\sum_{(x, \xi) \in \Lambda}\left|\left\langle f, M_{\xi} T_{x} g\right\rangle\right|^{2} & =\sum_{(x, \xi) \in \Lambda}\left|V_{g} f(x, \xi)\right|^{2} \\
& =\sum_{j \in \mathbf{Z}^{2 d}} \sum_{(x, \xi) \in \Lambda \cap Q+j}\left|V_{g} f(x, \xi)\right|^{2} \\
& \leq \sum_{j \in \mathbf{Z}^{2 d}} K \sup _{(x, \xi) \in Q+j}\left|V_{g} f(x, \xi)\right|^{2} \\
& =K\left\|V_{g} f\right\|_{W\left(\mathcal{C}, \ell^{2}\right)} \leq C K\|f\|_{2},
\end{aligned}
$$


where $C$ is the constant appearing in Proposition 2.

From now on, even though $\Lambda$ is assumed to be a sequence of points in $\mathbf{R}^{2 d}$ and not merely a subset (so repetitions of elements are allowed), for simplicity we will simply write $\Lambda \subset \mathbf{R}^{2 d}$.

\subsection{The Statement of the Density Theorem}

The Density Theorem for irregular Gabor systems can be stated as follows.

Theorem 13 (Density Theorem for Irregular Gabor Systems). Let $g \in L^{2}\left(\mathbf{R}^{d}\right)$ and let $\Lambda \subset \mathbf{R}^{2 d}$ be given. Then the Gabor system $\mathcal{G}(g, \Lambda)$ has the following properties.

(a) If $\mathcal{G}(g, \Lambda)$ is complete in $L^{2}\left(\mathbf{R}^{d}\right)$, then $0 \leq D^{-}(\Lambda) \leq D^{+}(\Lambda) \leq \infty$.

(b) If $\mathcal{G}(g, \Lambda)$ is a frame for $L^{2}\left(\mathbf{R}^{d}\right)$, then $1 \leq D^{-}(\Lambda) \leq D^{+}(\Lambda)<\infty$.

(c) If $\mathcal{G}(g, \Lambda)$ is a Riesz basis for $L^{2}\left(\mathbf{R}^{d}\right)$, then $D^{-}(\Lambda)=D^{+}(\Lambda)=1$. Moreover, $\Lambda$ is uniformly separated.

(d) If $\mathcal{G}(g, \Lambda)$ is a Riesz sequence in $L^{2}\left(\mathbf{R}^{d}\right)$, then $0 \leq D^{-}(\Lambda) \leq D^{+}(\Lambda) \leq 1$. Moreover, $\Lambda$ is uniformly separated.

The "critical density" $D^{-}(\Lambda)=D^{+}(\Lambda)=1$ is sometimes called the Nyquist density. Extensions of the Density Theorem to the case of multiple generators, i.e., Gabor systems of the form $\cup_{k=1}^{r} \mathcal{G}\left(g_{k}, \Lambda_{k}\right)$, are known, e.g., [42]. For simplicity of presentation we will discuss only the single generator case.

We consider each part of the Density Theorem in turn in the sections below. Of course, part (a) provides no information at all, but we state it in order to contrast it with part (a) of Theorems 3 and 10.

\subsection{Frames, Riesz Bases, and the Homogeneous Approximation Property}

For the one-dimensional case of $L^{2}(\mathbf{R})$, part (b) of the Density Theorem was first proved, but with extra hypotheses on $g$, by H. Landau [145]. Landau's technique is related to the energy concentration result for rectangular lattice Gabor frames proved by Daubechies [49, Theorem 3.5.2].

Parts (b) and (c) were proved for arbitrary $g \in L^{2}(\mathbf{R})$, but with extra hypotheses on $\Lambda$, by Ramanathan and Steger [158]. Specifically, Ramanathan and Steger only considered the case where $\Lambda$ is uniformly separated (and they also implicitly assume $D^{-}(\Lambda)>0$ ). However, not all irregular Gabor frames are uniformly separated. For example, the union of a frame and a Bessel sequence is again a frame, so if we let $g=\chi_{[0,1]^{d}}$, then $\mathcal{G}\left(g, \mathbf{Z}^{2 d} \cup \sqrt{2} \mathbf{Z}^{2 d}\right)$ is a frame, but this index set is not uniformly separated.

On the other hand, the fact that a Gabor Riesz sequence must be uniformly separated is well-known, although the only published proof we are aware of appears in [55].

Janssen [126] proved part (b) of Theorem 13 for "half irregular" $\Lambda \subset \mathbf{R}^{2}$, i.e., of the form $\Lambda=\alpha \mathbf{Z} \times \Gamma$ with $\Gamma$ irregular. Furthermore, Janssen's result actually applies to certain systems of Gabor molecules which need not be exact time-frequency shifts of a single generator, similar to Definition 8 below.

An important tool introduced by Ramanathan and Steger is the Homogeneous Approximation Property (HAP) for Gabor frames. The HAP is a fundamental result of independent interest, and essentially states that for any irregular Gabor frame $\mathcal{G}(g, \Lambda)$ in 
$L^{2}\left(\mathbf{R}^{d}\right)$, the rate of approximation of a Gabor frame expansion of a function $f$ is invariant under time-frequency shifts of $f$. This is surprising, since $\Lambda$ is not assumed to have any structure whatsoever, so there is no relationship between the specific frame elements used in the approximation of one time-frequency shift $M_{\eta} T_{u} f$ and those used in an approximation of another time-frequency shift of $f$.

Gröchenig and Razafinjatovo [98] adapted and refined the idea of the Homogeneous Approximation Property to obtain Nyquist density results for systems of translates in the space of bandlimited functions (but still with some extra hypotheses required, compare [109]).

Inspired by [98], Christensen, Deng, and Heil proved the HAP and parts (b) and (c) of Theorem 13 in [42], without any extra hypotheses (furthermore, the result was extended to higher dimensions and to multiple generators, and some other applications were made). The HAP was also applied to study the excess of Gabor frames in [11].

The form of the HAP used in [42] is the Weak HAP of the following definition. The distinction between and Weak and Strong HAPs was introduced in $[12,13]$. The Strong HAP implies the Weak HAP, and if $\mathcal{G}(g, \Lambda)$ is a Riesz basis then it follows as in [13, Theorem 10] that the Weak HAP implies the Strong HAP, cf. also [109].

Definition 5. Assume that $\mathcal{G}(g, \Lambda)=\left\{M_{\xi} T_{x} g\right\}_{(x, \xi) \in \Lambda}$ is a frame for $L^{2}\left(\mathbf{R}^{d}\right)$, and let $\tilde{\mathcal{G}}=\left\{\tilde{g}_{x, \xi}\right\}_{(x, \xi) \in \Lambda}$ denote its canonical dual frame. For each $r>0$ and $(u, \eta) \in \mathbf{R}^{2 d}$, set

$$
W(r, u, \eta)=\operatorname{span}\left\{\tilde{g}_{x, \xi}:(x, \xi) \in \Lambda \cap Q_{r}(u, \eta)\right\} .
$$

(a) We say that $\mathcal{G}$ possesses the Weak Homogeneous Approximation Property (Weak $H A P)$ if

$$
\begin{aligned}
\forall f \in L^{2}\left(\mathbf{R}^{d}\right), \quad \forall \varepsilon>0, \quad \exists R>0 \quad \text { such that } \quad \forall(u, \eta) \in \mathbf{R}^{2 d}, \\
\\
\operatorname{dist}\left(M_{\eta} T_{u} f, W(R, u, \eta)\right)<\varepsilon .
\end{aligned}
$$

(b) We say that $\mathcal{G}$ possesses the Strong Homogeneous Approximation Property (Strong $H A P)$ if

$$
\begin{aligned}
& \forall f \in L^{2}\left(\mathbf{R}^{d}\right), \quad \forall \varepsilon>0, \quad \exists R>0 \quad \text { such that } \quad \forall(u, \eta) \in \mathbf{R}^{2 d}, \\
& \left\|M_{\eta} T_{u} f-\sum_{(x, \xi) \in \Lambda \cap Q_{R}(u, \eta)}\left\langle M_{\eta} T_{u} f, M_{\xi} T_{x} g\right\rangle \tilde{g}_{x, \xi}\right\|_{2}<\varepsilon .
\end{aligned}
$$

The HAP introduced by Ramanathan and Steger in [158] is the Weak HAP given above for the case $d=1$ but with the roles of the frame and dual frame interchanged. It is proved there that if $\Lambda$ is uniformly separated and if $\mathcal{G}(g, \Lambda)$ is a frame then $\mathcal{G}(g, \Lambda)$ satisfies their version of the HAP. The argument of [158] does not show that a Strong HAP is satisfied. Ramanathan and Steger showed that the HAP implies a Comparison Theorem, which establishes an inequality between the density of any given Gabor frame satisfying the HAP and any given Riesz basis. The Density Theorem for frames and Riesz bases follows from this. A detailed exposition of the arguments of [158] appears in [107]. Gröchenig and Razafinjatovo's modified version of the HAP is essentially the Weak HAP given above, except defined for the setting of systems of translates in the space of bandlimited functions [98], and allowing finitely many generators.

It is proved in [42] that every Gabor frame $\mathcal{G}(g, \Lambda)$, without restrictions on $g$ or $\Lambda$ 
or the dimension, satisfies the Weak HAP given above, and in fact the argument actually shows that the Strong HAP is satisfied. The localized frame approach of [13] shows that even more is true: Gabor frames are $\ell^{2}$-localized with respect to any other lattice Gabor frame whose generator lies in $M^{1}$; this implies the Strong HAP as a corollary.

\subsection{Frames versus Complete Sequences}

Since the Beurling density of a rectangular lattice is $D^{-}(\alpha \mathbf{Z} \times \beta \mathbf{Z})=D^{+}(\alpha \mathbf{Z} \times \beta \mathbf{Z})=\frac{1}{\alpha \beta}$, Theorem 13 almost, but not quite, recovers Theorem 3. One trivial difference is in part (c) of the two theorems: The implications proceed in both directions in Theorem 3(c) but only in one direction in Theorem 13(c). For a counterexample to the converse direction in Theorem 13(c), take a Gabor frame $\mathcal{G}(g, \Lambda)$ that happens to be a Riesz basis and add a single point, say $\lambda$, to $\Lambda$. Then $\mathcal{G}(g, \Lambda \cup\{\lambda\})$ is a redundant frame, but the Beurling densities are the same, $D^{-}(\Lambda \cup\{\lambda\})=D^{+}(\Lambda \cup\{\lambda\})=1$. An open question is whether this is typical: If $\mathcal{G}(g, \Lambda)$ is a frame and $D^{ \pm}(\Lambda)=1$, must there exist a subset $\Lambda^{\prime} \subset \Lambda$ such that $\mathcal{G}\left(g, \Lambda^{\prime}\right)$ is a Riesz basis and $D^{ \pm}\left(\Lambda \backslash \Lambda^{\prime}\right)=0$ ?

On the other hand, the difference between Theorem 3(a) and Theorem 13(a) is much more striking. Ramanathan and Steger conjectured in [158] that Theorem 13(a) should be improvable to say that if $D^{+}(\Lambda)<1$, then $\mathcal{G}(g, \Lambda)$ is incomplete in $L^{2}(\mathbf{R})$. This was shown in [23] to be false: For any $\varepsilon>0$ there exists a function $g \in L^{2}(\mathbf{R})$ and a sequence $\Lambda \subset \mathbf{R}^{2}$ with $0<D^{+}(\Lambda)<\varepsilon$ such that $\mathcal{G}(g, \Lambda)$ is complete. The counterexample built in a fundamental way on the work of $\mathrm{H}$. Landau on the completeness of exponentials in $L^{2}(S)$ where $S$ is a finite union of intervals [142]. Another counterexample, in which $\Lambda$ is a subset of a lattice, was given by Wang [183].

Zalik showed in $[191,192]$ that there exist $g \in L^{2}(\mathbf{R})$ and countable subsets $\Gamma \subset \mathbf{R}$ such that the set of translates $\left\{T_{x} g\right\}_{x \in \Gamma}$ is complete in $L^{2}(\mathbf{R})$. Such a set of translates is an irregular Gabor system of the form $\mathcal{G}(g, \Lambda)$ where $\Lambda=\Gamma \times\{0\}$. The lower Beurling density of $\Lambda=\Gamma \times\{0\}$ is zero, but in Zalik's construction, the upper Beurling density of $\Lambda$ as a subset of $\mathbf{R}^{2}$ is infinite. In contrast, Olevskii and Ulanovskii showed in $[154,155]$ that there exists a countable set $\Gamma$ which is a bounded perturbation of the integers $\mathbf{Z}$ and a function $g \in L^{2}(\mathbf{R})$ such that $\left\{T_{x} g\right\}_{x \in \Gamma}$ is complete in $L^{2}(\mathbf{R})$. Thus $\Lambda=\Gamma \times\{0\}$ satisfies $D^{-}(\Lambda)=D^{+}(\Lambda)=0$ for this example.

By the Density Theorem, no sequence of pure translations $\left\{T_{x} g\right\}_{x \in \Gamma}$ of an $L^{2}$ function can ever form a frame or Riesz basis for $L^{2}\left(\mathbf{R}^{d}\right)$, because the lower density of $\Lambda=\Gamma \times\{0\}$ is $D^{-}(\Lambda)=0$, cf. [42]. The fact that no sequence of translates can form a Riesz basis for $L^{2}\left(\mathbf{R}^{d}\right)$ was earlier proved by Olson and Zalik [156]. Moreover, it was conjectured there that no sequence $\left\{T_{x} g\right\}_{x \in \Gamma}$ can form a Schauder basis for $L^{2}\left(\mathbf{R}^{d}\right)$, but this conjecture remains open. Partial results on this conjecture and for irregular Gabor Schauder bases were obtained in [55]. Lattice Gabor Schauder bases for $L^{2}\left(\mathbf{R}^{d}\right)$ were characterized in [111].

An example of $\mathcal{G}(g, \Lambda)$ that is complete and satisfies $D^{ \pm}(\Lambda)=0$ but which is not a system of pure translations was constructed in [160].

\subsection{Riesz Sequences}

In the lattice setting, we saw that part (d) of both Theorems 3 and 10 was a consequence of part (b) and the Duality Principle. For irregular Gabor frames, there is no known direct analogue of the Duality Principle. Indeed, not only is there no obvious replacement for the notion of the adjoint lattice, but the canonical dual frame need no longer be itself a Gabor frame. Very little was known about the structure of the dual of an irregular Gabor frame 
until the advent of localized frames, which are discussed in Section 6 below. Thus, part (d) of Theorem 13 is perhaps especially interesting in light of the lack of a Duality Principle. While it is possible to prove part (d) using the types of techniques that appear in [158, 42], it does not appear explicitly in those article. The first proof of part (d) appears in [13], as a consequence of the abstract results for localized frames proved in [12].

\subsection{Existence}

It follows from the coorbit theory of Feichtinger and Gröchenig $[64,65,86]$ that if $g$ lies in the modulation space $M^{1}$, then $\mathcal{G}(g, \Lambda)$ will be a frame for every sufficiently dense set of indices $\Lambda$.

For the case $d=1$, the results of Lyubarskii [150] and Seip and Wallstén [164, 168] on the density of sets of sampling and interpolation in the Bargmann-Fock space imply the following.

Theorem 14. Let $\varphi(t)=2^{1 / 4} e^{-\pi t^{2}}$ be the Gaussian window and $\Lambda$ be any sequence in $\mathbf{R}^{2}$. Then the following two statements are equivalent.

(a) $\mathcal{G}(\varphi, \Lambda)$ is a frame for $L^{2}(\mathbf{R})$.

(b) There exists a uniformly separated subset $\Lambda^{\prime}$ of $\Lambda$ such that

$$
1<D^{-}\left(\Lambda^{\prime}\right) \leq D^{+}(\Lambda)<\infty .
$$

The following two statements are also equivalent.

(a') $\mathcal{G}(\varphi, \Lambda)$ is a Riesz sequence in $L^{2}(\mathbf{R})$.

(b') $\Lambda$ is uniformly separated and $D^{+}(\Lambda)<1$.

The proof relies on the fact that the Bargmann transform of any $f \in L^{2}\left(\mathbf{R}^{d}\right)$ is an analytic function. The Bargmann transform is closely related to the short-time Fourier transform $V_{\varphi} f$ using a Gaussian window, e.g., see [89, Section 3.4]. We provide in Appendix A a discussion of the relationship between properties of Gabor systems generated by the Gaussian and sets of sampling and interpolation in the Bargmann-Fock space.

We refer to Seip's text [167] for a recent discussion of results on sampling and interpolation, and to [146] for recent results in higher dimensions.

Remark 3. If we allow the possibility that $\inf \left\|f_{n}\right\|=0$, then it is easy to construct a frame $\mathcal{F}=\left\{f_{n}\right\}_{n \in \mathbf{N}}$ such that no subset of $\mathcal{F}$ is a Riesz basis. The first example of a frame which is norm-bounded below $\left(\inf \left\|f_{n}\right\|>0\right.$ ) but which contains no Riesz bases was given by Seip [166]. In that article, Seip obtained a variety of deep results related to the question of when a system of nonharmonic complex exponentials $\left\{e^{2 \pi i \lambda_{n} t}\right\}_{n \in \mathbf{N}}$ that forms a frame for $L^{2}[0,1]$ will contain a Riesz basis, or when a Riesz sequence of exponentials can be extended to form a frame for $L^{2}[0,1]$.

Casazza and Christensen also constructed a frame which is norm-bounded below and which does not contain a Riesz basis in [31]. They furthermore showed in [32] that this frame contains no subsets which form a Schauder basis. In [10] it is shown that this frame has infinite excess and hence an infinite subset may be removed yet leave a complete set. However, whenever infinitely many elements are removed from this frame, the resulting subset is not a frame.

It is interesting to note that Gabor frames with Gaussian windows provide additional examples of frames which do not contain Riesz bases as subsets. For, suppose that $\mathcal{G}(\varphi, \Lambda)$ is a frame for $L^{2}(\mathbf{R})$ and there exists some subset $\Delta \subset \Lambda$ such that $\mathcal{G}(\varphi, \Delta)$ is a Riesz basis 
for $L^{2}(\mathbf{R})$. Then $\mathcal{G}(\varphi, \Delta)$ would also be a Riesz sequence and hence by Theorem 14 must satisfy $D^{+}(\Delta)<1$. But, by the Density Theorem, a Riesz basis requires $D^{ \pm}(\Delta)=1$, which is a contradiction.

\section{Localized Frames and Their Implications for Gabor Systems}

Recently, Gröchenig [91] and Balan, Casazza, Heil, and Z. Landau [12, 13] independently realized that many aspects of Gabor frame theory are not dependent on the rigid timefrequency shift structure of Gabor frame elements, but hold in a much broader context. The concept of localized frames was independently introduced in these two sets of article. Among other results, Gröchenig showed in his elegant article that localized frames are frames not merely for the underlying Hilbert space $H$ but also for an entire family of associated Banach spaces. Thus localized frames are robust in the sense that they are not limited merely to the Hilbert space setting, and the frame coefficients encode a much broader range of information than just the Hilbert space norm. For the case of Gabor frames, these associated spaces are precisely the modulation spaces discussed in Section 2.3. Gröchenig also obtained results on the structure of the canonical dual of a localized frame. Some additional article on localized frames include [90, 46, 28, 85, 75].

The focus in the article $[12,13]$ is on the excess and overcompleteness of localized frames, the connection between density and redundancy, the relationship between frame bounds and density, and the structure of the canonical dual frame of a localized frame. Among other results, it is shown that the Density Theorem and the Homogeneous Approximation Properties are not merely results about Gabor frames and time-frequency shifts but extend to the much more general situation of localized frames. Moreover, new consequences follow even for Gabor frames. For example, part (d) of Theorem 13 is first proved in [13]. We discuss some other consequences of these article for Gabor frames in this section. All of the results discussed in this section are special cases of more general results proved in $[12,13]$ for abstract localized frames, but in keeping with the setting of this article we will formulate the definitions and results for the specific concrete setting of Gabor systems in $L^{2}\left(\mathbf{R}^{d}\right)$, and refer to the original article for the abstract formulations.

\subsection{Density Reinterpreted: Measure and Relative Measure}

The lower and upper Beurling densities of a sequence $\Lambda$ are only the extremes of the possible densities that we could naturally assign to $\Lambda$. In particular, instead of taking the infimum or supremum over all possible centers in Definition 4, we could choose one specific sequence of centers, and instead of computing the liminf or limsup we could consider the limit with respect to some ultrafilter (for a brief review of ultrafilters, see Appendix B). The different possible choices of ultrafilters and sequences of centers provides us with the following natural collection of definitions of density [12].

Definition 6 (Density). Let $\Lambda \subset \mathbf{R}^{2 d}$ with $D^{+}(\Lambda)<\infty$ be given. Given a free ultrafilter $p$ and a sequence $c=\left(c_{N}\right)_{N \in \mathbf{N}}$ of points in $\mathbf{R}^{2 d}$, the Beurling density of $\Lambda$ with respect to $p$ and $c$ is

$$
D(\Lambda ; p, c)=p-\lim _{N \in \mathbf{N}} \frac{\left|\Lambda \cap Q_{N}\left(c_{N}\right)\right|}{N^{2 d}} .
$$

We have $0 \leq D^{-}(\Lambda) \leq D(\Lambda ; p, c) \leq D^{+}(\Lambda) \leq \infty$. Furthermore, there exists a free 
ultrafilter $p$ and sequence of centers $c$ such that $D(\Lambda ; p, c)=D^{-}(\Lambda)$, and similarly for the upper Beurling density.

The notion of the measure of an abstract localized frame was introduced in [12]. For the case of Gabor systems, it can be defined as follows. For simplicity of notation, we will use the following shorthand for a Gabor frame $\mathcal{G}(g, \Lambda)$, its canonical dual $\tilde{\mathcal{G}}$, and their respective elements:

$$
\mathcal{G}=\mathcal{G}(g, \Lambda)=\left\{g_{\lambda}\right\}_{\lambda \in \Lambda} \quad \text { and } \quad \tilde{\mathcal{G}}=\left\{\tilde{g}_{\lambda}\right\}_{\lambda \in \Lambda} .
$$

Note that while $g_{\lambda}$ denotes a time-frequency shift of $g$, in general the functions $\tilde{g}_{\lambda}$ need not be time-frequency shifts of a single function (compare Section 6.3 below).

Definition 7 (Measure). Let $g \in L^{2}\left(\mathbf{R}^{d}\right)$ and $\Lambda \subset \mathbf{R}^{2 d}$ with $0<D^{-}(\Lambda) \leq D^{+}(\Lambda)<\infty$ be such that $\mathcal{G}(g, \Lambda)$ is a frame for $L^{2}\left(\mathbf{R}^{d}\right)$.

(a) The upper measure of $\mathcal{G}$ is

$$
\mathcal{M}^{+}(\mathcal{G})=\limsup _{N \rightarrow \infty} \sup _{z \in \mathbf{R}^{2 d}} \frac{1}{\left|\Lambda \cap Q_{N}(z)\right|} \sum_{\lambda \in \Lambda \cap Q_{N}(z)}\left\langle g_{\lambda}, \tilde{g}_{\lambda}\right\rangle .
$$

(b) The lower measure of $\mathcal{G}$ is

$$
\mathcal{M}^{-}(\mathcal{G})=\liminf _{N \rightarrow \infty} \inf _{z \in \mathbf{R}^{2 d}} \frac{1}{\left|\Lambda \cap Q_{N}(z)\right|} \sum_{\lambda \in \Lambda \cap Q_{N}(z)}\left\langle g_{\lambda}, \tilde{g}_{\lambda}\right\rangle .
$$

(c) Let $p$ be a free ultrafilter and let $c=\left(c_{N}\right)_{N \in \mathbf{N}}$ be any sequence of points in $\mathbf{R}^{2 d}$. Then the measure of $\mathcal{G}$ with respect to $p$ and $c$ is

$$
\mathcal{M}(\mathcal{G} ; p, c)=p-\lim _{N \in \mathbf{N}} \frac{1}{\left|\Lambda \cap Q_{N}\left(c_{N}\right)\right|} \sum_{\lambda \in \Lambda \cap Q_{N}\left(c_{N}\right)}\left\langle g_{\lambda}, \tilde{g}_{\lambda}\right\rangle
$$

Thus, the measure is in some sense an average trace of the "cross-Grammian matrix" $\left[\left\langle g_{\lambda}, \tilde{g}_{\mu}\right\rangle\right]_{\lambda, \mu \in \Lambda}$. The notion of localization of a frame corresponds to decay conditions on this or other appropriate matrices; each choice of decay condition gives a different notion of localization. However, to make this precise, instead of indexing by $\Lambda$ (which has no structure), the matrix is indexed by an additive subgroup of $\mathbf{R}^{2 d}$ (namely, $\alpha \mathbf{Z}^{d} \times \beta \mathbf{Z}^{d}$ ), and indices in $\Lambda$ are associated with indices in this subgroup by rounding off to a near element of $\alpha \mathbf{Z}^{d} \times \beta \mathbf{Z}^{d}$. In particular, the fact that there exist discrete additive subgroups of $\mathbf{R}^{2 d}$ is a hidden, but essential, element of many of the results relating to localization for Gabor frames proved in $[12,13]$.

Remark 4. (a) There exists a free ultrafilter $p$ and sequence of centers $c$ such that $\mathcal{M}(\mathcal{G} ; p, c)=\mathcal{M}^{-}(\mathcal{G})$, and similarly for the upper measure.

(b) Since $\left\langle g_{\lambda}, \tilde{g}_{\lambda}\right\rangle=\left\|S^{-1 / 2} g_{\lambda}\right\|_{2}$ and since $S^{-1 / 2}(\mathcal{G}(g, \Lambda))$ is a Parseval frame, we have $0 \leq\left\langle g_{\lambda}, \tilde{g}_{\lambda}\right\rangle \leq 1$ for every $\lambda \in \Lambda$. Hence for any free ultrafilter $p$ and any sequence of centers $c$, we have

$$
0 \leq \mathcal{M}^{-}(\mathcal{G}) \leq \mathcal{M}(\mathcal{G} ; p, c) \leq \mathcal{M}^{+}(\mathcal{G}) \leq 1
$$

Localization and the measure of a frame with respect to frames other than the canonical dual frame are equally important notions. In particular, intrinsic or self-localization compares a frame to itself [75]. 
The notion of measure can also be defined for frame sequences, but in this case the fact that the frame sequences can have distinct closed spans must be taken into account. Therefore for frame sequences we speak of the relative measure of the frame sequence with respect to the reference sequence (see [12] for details).

We will give a precise formulation of localization for Gabor frames in Section 6.4. First, however, we will discuss the consequences and implications of localization for Gabor frames. In particular, we have the following new interpretation of the density of a Gabor frame [13, Theorem 3].

Theorem 15 (Density-Measure). Let $g \in L^{2}\left(\mathbf{R}^{d}\right)$ and $\Lambda \subset \mathbf{R}^{2 d}$ be such that $\mathcal{G}(g, \Lambda)$ is a Gabor frame for $L^{2}\left(\mathbf{R}^{d}\right)$. Then $D^{+}(\Lambda)<\infty$, and the following statements hold.

(a) For any free ultrafilter $p$ and any sequence of centers $c=\left(c_{N}\right)_{N \in \mathbf{N}}$ in $\mathbf{R}^{d}$, we have

$$
\mathcal{M}(\mathcal{G} ; p, c)=\frac{1}{D(\Lambda ; p, c)}
$$

and consequently $p$ - $\lim _{N \in \mathbf{N}} \frac{1}{N^{2 d}} \sum_{\lambda \in \Lambda \cap Q_{N}\left(c_{N}\right)}\left\langle g_{\lambda}, \tilde{g}_{\lambda}\right\rangle=1$.

(b) $\mathcal{M}^{-}(\mathcal{G})=\frac{1}{D^{+}(\Lambda)}$ and $\mathcal{M}^{+}(\mathcal{G})=\frac{1}{D^{-}(\Lambda)}$.

This result is perhaps surprising in the sense that the density is determined entirely by the index set $\Lambda$ alone, while the measure is determined by averages of inner products of frame elements with their corresponding dual frame elements.

As immediate consequences we obtain the Density Theorem for irregular Gabor frames (Theorem 13), as well as new implications on the relationship between density, measure, and frame bounds. To see how Theorem 15 implies the Density Theorem, assume that $\mathcal{G}(g, \Lambda)$ is a frame for $L^{2}\left(\mathbf{R}^{d}\right)$. Then Theorem 15 gives $D^{+}(\Lambda)<\infty$, and since we already know that $0 \leq \mathcal{M}^{-}(\mathcal{G}) \leq \mathcal{M}^{+}(\mathcal{G}) \leq 1$, part (b) of Theorem 15 implies as an immediate consequence that $1 \leq D^{-}(\mathcal{G}) \leq D^{+}(\mathcal{G})<\infty$. This proves part (b) of Theorem 13 .

If $\mathcal{G}(g, \Lambda)$ is a Riesz basis then $\left\langle g_{\lambda}, \tilde{g}_{\lambda}\right\rangle=1$ for every $\lambda \in \Lambda$, and so $\mathcal{M}^{ \pm}(\mathcal{G})=1$. Hence Theorem 15 implies that $D^{ \pm}(\Lambda)=1$. This proves part (c) of Theorem 13 .

The final portion of Theorem 13, concerning the density of Riesz sequences, follows from refinements of the results that we have discussed for the case of frame sequences, cf. [13, Theorem 4].

Moreover, we do not simply recover the Density Theorem, but we obtain further immediate new consequences. One very easy consequence is the following result relating the frame bounds, the density of the index set, and the norm of the window, cf. also [138].

Corollary 2 (Density-Frame Bounds). Let $g \in L^{2}\left(\mathbf{R}^{d}\right)$ and $\Lambda \subset \mathbf{R}^{2 d}$ be such that $\mathcal{G}(g, \Lambda)$ is a Gabor frame for $L^{2}\left(\mathbf{R}^{d}\right)$ with frame bounds $A, B$. Then the following statements hold.

(a) $\quad A \leq D^{-}(\Lambda)\|g\|_{2}^{2} \leq D^{+}(\Lambda)\|g\|_{2}^{2} \leq B$.

(b) If $\mathcal{G}(g, \Lambda)$ is a tight frame, then $\Lambda$ has uniform Beurling density, i.e., $D^{-}(\Lambda)=$ $D^{+}(\Lambda)$, and furthermore $A=D^{ \pm}(\Lambda)\|g\|_{2}^{2}$.

Proof. (a) Let $S$ be the frame operator for $\mathcal{G}$. Then $A I \leq S \leq B I$, so

$$
\left\langle g_{\lambda}, \tilde{g}_{\lambda}\right\rangle=\left\langle g_{\lambda}, S^{-1} g_{\lambda}\right\rangle \leq \frac{1}{A}\left\langle g_{\lambda}, g_{\lambda}\right\rangle=\frac{1}{A}\left\|g_{\lambda}\right\|^{2}=\frac{1}{A}\|g\|_{2}^{2} .
$$


Hence, taking averages we see that

$$
\begin{aligned}
\mathcal{M}(\mathcal{G} ; p, c) & =p-\lim _{N \in \mathbf{N}} \frac{1}{\left|\Lambda \cap Q_{N}\left(c_{N}\right)\right|} \sum_{\lambda \in \Lambda \cap Q_{N}\left(c_{N}\right)}\left\langle g_{\lambda}, \tilde{g}_{\lambda}\right\rangle \\
& \leq \frac{1}{A} p_{N \in \mathbf{N}} \frac{1}{\left|\Lambda \cap Q_{N}\left(c_{N}\right)\right|} \sum_{\lambda \in \Lambda \cap Q_{N}\left(c_{N}\right)}\|g\|_{2}^{2}=\frac{1}{A}\|g\|_{2}^{2} .
\end{aligned}
$$

Since this is true for every free ultrafilter $p$ and choice of centers $c$, we conclude that

$$
\frac{1}{D^{-}(\Lambda)}=\mathcal{M}^{+}(\mathcal{G}) \leq \frac{1}{A}\|g\|_{2}^{2}
$$

The other inequality is similar.

(b) This is an immediate consequence of part (a), since if the frame is tight, then by definition we can take $A=B$.

Remark 5. It is interesting to see what these results say for the case of lattice Gabor frames, and for rectangular lattices in particular. Consider $\Lambda=\alpha \mathbf{Z} \times \beta \mathbf{Z}$. If $\mathcal{G}(g, \Lambda)$ is a frame, then its dual frame is also a Gabor frame with respect to the same lattice, i.e., it has the form $\tilde{\mathcal{G}}=\mathcal{G}(\tilde{g}, \Lambda)$ with $\tilde{g}=S^{-1} g$. In this case, we have

$$
\left\langle g_{\lambda}, \tilde{g}_{\lambda}\right\rangle=\langle g, \tilde{g}\rangle, \quad \lambda \in \Lambda .
$$

In fact, the cross-Grammian matrix $\left[\left\langle g_{\lambda}, \tilde{g}_{\mu}\right\rangle\right]_{\lambda, \mu \in \Lambda}$ is actually a Toeplitz matrix. Furthermore, the measure of the frame is simply

$$
\begin{aligned}
\mathcal{M}(\mathcal{G} ; p, c) & =p-\lim _{N \in \mathbf{N}} \frac{1}{\left|\Lambda \cap Q_{N}\left(c_{N}\right)\right|} \sum_{\lambda \in \Lambda \cap Q_{N}\left(c_{N}\right)}\left\langle g_{\lambda}, \tilde{g}_{\lambda}\right\rangle \\
& =p-\lim _{N \in \mathbf{N}} \frac{1}{\left|\Lambda \cap Q_{N}\left(c_{N}\right)\right|} \sum_{\lambda \in \Lambda \cap Q_{N}\left(c_{N}\right)}\langle g, \tilde{g}\rangle=\langle g, \tilde{g}\rangle .
\end{aligned}
$$

Therefore, by Theorem 15, we conclude that

$$
\langle g, \tilde{g}\rangle=\mathcal{M}(\mathcal{G} ; p, c)=\frac{1}{D(\Lambda ; p, c)}=\alpha \beta
$$

the final equality following from the fact that $\Lambda=\alpha \mathbf{Z} \times \beta \mathbf{Z}$. In other words, we recover the special case of the Wexler-Raz biorthogonality relations in Equation (4) corresponding to $k=k^{\prime}, n=n^{\prime}$. In this sense, Theorem 15 might be considered a type of limited Wexler-Raz theorem for irregular Gabor frames.

Furthermore, for the case of a rectangular lattice $\Lambda=\alpha \mathbf{Z} \times \beta \mathbf{Z}$, the frame bound relationships in Corollary 2(c) become

$$
A \leq \frac{\|g\|_{2}^{2}}{\alpha \beta} \leq B
$$

which was obtained by Daubechies in [48]. It is interesting that Theorem 15 generalizes this result without relying on operator theory or von Neumann algebras. 


\subsection{Redundancy}

There is a folklore that the density of a Gabor frame is directly related to the redundancy of the frame. We will show in this section that only very weak results related to the quantification of this folklore exist. These results are again the implications for Gabor frames of results that hold for abstract localized frames.

For certain $g$ and certain values of $\alpha \beta$, we can easily see that there is a natural relation between the redundancy of a rectangular lattice Gabor frame $\mathcal{G}(g, \alpha \mathbf{Z} \times \beta \mathbf{Z})$ and Beurling density $\frac{1}{\alpha \beta}$ of the lattice $\alpha \mathbf{Z} \times \beta \mathbf{Z}$. For example, if $g=\chi_{[0,1]}, \alpha=\frac{1}{2}, \beta=1$, then we can write

$$
\mathcal{G}\left(\chi_{[0,1]}, \frac{1}{2} \mathbf{Z} \times \mathbf{Z}\right)=\mathcal{G}\left(\chi_{[0,1]}, \mathbf{Z} \times \mathbf{Z}\right) \cup \mathcal{G}\left(\chi_{[0,1]},\left(\mathbf{Z}+\frac{1}{2}\right) \times \mathbf{Z}\right) .
$$

Thus, the frame $\mathcal{G}\left(\chi_{[0,1]}, \frac{1}{2} \mathbf{Z} \times \mathbf{Z}\right)$ can be divided into two disjoint subsets that are each orthonormal bases for $L^{2}(\mathbf{R})$. Perhaps stemming from examples such as these, the quantity $\frac{1}{\alpha \beta}$ is sometimes called the redundancy of a frame $\mathcal{G}(g, \alpha \mathbf{Z} \times \beta \mathbf{Z})$, or it is said that $\mathcal{G}(g, \alpha \mathbf{Z} \times \beta \mathbf{Z})$ is $\frac{1}{\alpha \beta}$-times overcomplete. However, this is merely terminology, and we may ask if there is a quantification of the meaning of "redundancy" and its connection to the density $\frac{1}{\alpha \beta}$ of the lattice $\alpha \mathbf{Z} \times \beta \mathbf{Z}$.

Intuitively, we might hope that redundancy means that we could divide a rectangular lattice Gabor frame $\mathcal{G}(g, \alpha \mathbf{Z} \times \beta \mathbf{Z})$ into " $\frac{1}{\alpha \beta}$ " Riesz bases. Setting aside for the moment the obvious problem that $\frac{1}{\alpha \beta}$ need not be integer, we can see that this cannot be literally true. For example, consider the frame $\mathcal{G}\left(\varphi, \frac{1}{2} \mathbf{Z} \times \mathbf{Z}\right)$ generated by the Gaussian function $\varphi(t)=2^{1 / 4} e^{-\pi t^{2}}$. By the Density Theorem for irregular Gabor frames, if there exists a subset $\Lambda \subset \frac{1}{2} \mathbf{Z} \times \mathbf{Z}$ such that $\mathcal{G}(\varphi, \Lambda)$ is a Riesz basis for $L^{2}(\mathbf{R})$, then necessarily $D^{ \pm}(\Lambda)=1$. However, by Lyubarskii and Seip-Wallstén, we know that $\mathcal{G}(\varphi, \Lambda)$ can be a frame only when $D^{-}(\Lambda)>1$. Hence $\mathcal{G}\left(\varphi, \frac{1}{2} \mathbf{Z} \times \mathbf{Z}\right)$, and indeed any Gabor frame generated by the Gaussian function, contains no subsets that are Riesz bases for $L^{2}(\mathbf{R})$. On the other hand, we can certainly write

$$
\mathcal{G}\left(\varphi, \frac{1}{2} \mathbf{Z} \times \mathbf{Z}\right)=\mathcal{G}(\varphi, \mathbf{Z} \times \mathbf{Z}) \cup \mathcal{G}\left(\varphi,\left(\mathbf{Z}+\frac{1}{2}\right) \times \mathbf{Z}\right) .
$$

However, the two subsets $\mathcal{G}(\varphi, \mathbf{Z} \times \mathbf{Z}), \mathcal{G}\left(\varphi,\left(\mathbf{Z}+\frac{1}{2}\right) \times \mathbf{Z}\right)$ are neither frames nor Riesz bases for $L^{2}(\mathbf{R})$. Instead, each of them is overcomplete by a single element (meaning that one but not two elements may be removed and still leave a complete set). If a single element is removed, the resulting set is exact, but it is neither a frame nor even a Schauder basis, cf. [73, p. 168], [129].

Rather than trying to split into $N$ Riesz bases, we might hope that the value $R=$ $\frac{1}{D^{ \pm}(\Lambda)}$ for a general Gabor frame $\mathcal{G}(g, \Lambda)$ is quantifying the redundancy in the sense that the frame is "almost $R$-times overcomplete," meaning that we should be able to remove a subset $J$ of $\Lambda$ with density $D^{ \pm}(J)=R-1-\varepsilon$ and still leave a frame. In other words, we might expect to be able to find a subset $I \subset \Lambda$ with $1 \leq D^{ \pm}(I) \leq 1+\varepsilon$ such that $\mathcal{G}(g, I)$ is still a frame. The best partial result in this direction seems to be the following $[12,13]$. Some concentration of the generator $g$ in the time-frequency plane is required in this result, specifically that $g$ lies in the modulation space $M^{1}$ defined in Section 2.3.

Theorem 16. Assume that $\mathcal{G}(g, \Lambda)$ is a Gabor frame for $L^{2}\left(\mathbf{R}^{d}\right)$. If $D^{-}(\Lambda)>1$ and $g \in M^{1}\left(\mathbf{R}^{d}\right)$, then there exists $J \subset \Lambda$ with $D^{+}(J)=D^{-}(J)>0$ such that $\mathcal{G}(g, \Lambda \backslash J)$ is a Gabor frame for $L^{2}\left(\mathbf{R}^{d}\right)$. 
Thus, not only does the frame $\mathcal{G}(g, \Lambda)$ have infinite excess, but a subset with some positive (but possibly very small) density can be removed yet still leave a frame.

In general, questions about dividing a frame into nonredundant subparts are extremely difficult. Consider the following conjecture.

Conjecture 1 (Feichtinger Conjecture). If $\mathcal{F}=\left\{f_{i}\right\}_{i \in I}$ is a frame and $\inf _{i}\left\|f_{i}\right\|>0$, then $\mathcal{F}$ can be written as a union of finitely many Riesz sequences.

Recently, it has been shown by Casazza and Tremain that the Feichtinger Conjecture is equivalent to the Kadison-Singer Conjecture, which is the deepest open problem in operator theory today [39].

For the case of Gabor frames $\mathcal{G}(g, \Lambda)$, it is known that the Feichtinger Conjecture is true if either: (a) $g \in M^{1}\left(\mathbf{R}^{d}\right)$ and $\Lambda$ is arbitrary [13], or (b) $g \in L^{2}(\mathbf{R})$ and $\Lambda=\alpha \mathbf{Z} \times \beta \mathbf{Z}$ with $\alpha \beta$ rational [33]; see also [28]. It was earlier proved by Gröchenig that the Feichtinger Conjecture is true if $g \in M_{v_{s}}^{\infty}\left(\mathbf{R}^{d}\right)$ where $v_{s}(x, \omega)=(1+|x|+|\omega|)^{s}$ and $s>2 d$ [90]; this hypothesis implies that $g \in M^{1}\left(\mathbf{R}^{d}\right)$. The Feichtinger Conjecture for arbitrary irregular Gabor frames $\mathcal{G}(g, \Lambda)$ remains open today.

The redundancy of Gabor frames is still far from being understood.

\subsection{Structure of the Canonical Dual Frame}

As noted before, the canonical dual of an irregular Gabor frame need not itself be a Gabor frame. In this section we discuss the impact of results on abstract localized frames on the structure of the canonical dual frame of an irregular Gabor frame.

It follows from abstract frame theory that if $\mathcal{G}(g, \Lambda)$ is a frame for $L^{2}\left(\mathbf{R}^{d}\right)$ then the canonical dual frame is some sequence $\tilde{\mathcal{G}}=\left\{\tilde{g}_{x, \xi}\right\}_{(x, \xi) \in \Lambda}$ of functions in $L^{2}\left(\mathbf{R}^{d}\right)$. However, until [91, 12, 13], essentially nothing was known about the properties of the elements of $\mathcal{G}$. Thus, it was a major advance when Gröchenig proved in [91] that if the short-time Fourier transform (STFT) of $g$ has a polynomial or exponential rate of decay, then the STFT of the elements of the dual frame will also possess a polynomial or exponential decay, and furthermore the element $\tilde{g}_{x, \xi}$ will be localized in the time-frequency plane around the point $(x, \xi)$. A similar result was obtained in [13], but instead $\ell^{p}$ and other types of localization were the focus there. Fornasier and Gröchenig [75] showed that the essential feature of these results is the existence of an inverse-closed Banach $*$-algebra of matrices - each such Banach algebra yields a structure result for the dual frame.

To state the result from [13] precisely, we need the following definition of Gabor molecules. For simplicity, our definition uses STFTs with respect to the Gaussian window $\varphi(t)=2^{d / 4} e^{-\pi t \cdot t}$, but any nonzero $M^{1}$ window can be used, see [13, Lemma 3]. The terminology "molecule" arises from the convention that the generator $g$ of a Gabor system $\mathcal{G}(g, \Lambda)$ is often referred to as an "atom."

Definition 8. Let $\Lambda \subset \mathbf{R}^{2 d}$ and $f_{\lambda} \in L^{2}\left(\mathbf{R}^{d}\right)$ for $\lambda \in \Lambda$ be given. Then $\mathcal{F}=\left\{f_{\lambda}\right\}_{\lambda \in \Lambda}$ is a set of Gabor molecules if there exists an envelope function $\Gamma \in W\left(\mathcal{C}, \ell^{2}\right)$ such that

$$
\forall \lambda \in \Lambda, \quad \forall z \in \mathbf{R}^{2 d}, \quad\left|V_{\varphi} f_{\lambda}(z)\right| \leq \Gamma(z-\lambda) .
$$

Remark 6. The short-time Fourier transform of a time-frequency shift of a function $f \in L^{2}\left(\mathbf{R}^{d}\right)$ satisfies the equality

$$
\forall(x, \xi) \in \mathbf{R}^{2 d}, \quad \forall(u, \eta) \in \mathbf{R}^{2 d}, \quad\left|V_{\varphi}\left(M_{\xi} T_{x} f\right)(u, \eta)\right|=\left|V_{\varphi} f(u-x, \eta-\xi)\right|,
$$


and furthermore $V_{\varphi} f \in W\left(\mathcal{C}, \ell^{2}\right)$ [89, Theorem 12.2.1]. In light of this, we see that Equation (6.2) essentially says that the STFT of a Gabor molecule element $f_{\lambda}$ is concentrated around the point $\lambda$ in the time-frequency plane. Thus, while the functions $f_{\lambda}$ are not exact time-frequency shifts of a single function, they are "almost" time-frequency shifts in the sense that they are concentrated at the appropriate point $\lambda$ in the time-frequency plane, with a common envelope of concentration (determined by $\Gamma$ ). Thus, a set of Gabor molecules is essentially a perturbation of a Gabor system (with control on the amount of perturbation).

The following result is [13, Theorem 7]; compare also the results in [91, Theorem 20] and [75]. Note that this result applies to frame sequences as well as frames.

Theorem 17 (Structure Theorem). Let $g \in M^{1}\left(\mathbf{R}^{d}\right)$ and $\Lambda \subset \mathbf{R}^{2 d}$ be such that $\mathcal{G}(g, \Lambda)$ is a Gabor frame sequence in $L^{2}\left(\mathbf{R}^{d}\right)$, with canonical dual frame sequence $\tilde{\mathcal{G}}=\left\{\tilde{g}_{\lambda}\right\}_{\lambda \in \Lambda}$. Then the following statements hold:

(a) $\tilde{g}_{\lambda} \in M^{1}\left(\mathbf{R}^{d}\right)$ for all $\lambda \in \Lambda$,

(b) $\sup _{\lambda}\left\|\tilde{g}_{\lambda}\right\|_{M^{1}}<\infty$, and

(c) $\tilde{\mathcal{G}}$ is a set of Gabor molecules with respect to an envelope function $\Gamma \in W\left(\mathcal{C}, \ell^{1}\right)$.

Furthermore, the same conclusions hold when $\tilde{\mathcal{G}}$ is replaced by the canonical Parseval frame $S^{-1 / 2}(\mathcal{G}(g, \Lambda))$.

The proof of the Structure Theorem relies on the following type of noncommutative Wiener's lemma that was independently proved by Gohberg, Kaashoek, and Woerdeman [82], Baskakov [16], and Sjöstrand [169]. Compare Gröchenig's use of Jaffard's Lemma $[117]$ to establish the related polynomial version of the Structure Theorem, as well as the use of noncommutative Wiener's lemmas for lattice Gabor frames in [89, Section 13.3], [97]. We also refer to the new results in $[92,99,172]$ for a deeper understanding of the role of Wiener's lemmas in time-frequency analysis.

Theorem 18. Let $G=\alpha \mathbf{Z}^{d} \times \beta \mathbf{Z}^{d}$. If an infinite matrix $A=\left[a_{i j}\right]_{i, j \in G}$ satisfies:

(a) there exists $r \in \ell^{1}(G)$ such that $\left|a_{i j}\right| \leq r(i-j)$ for all $i, j \in G$, and

(b) $A$ is a continuous invertible map of $\ell^{2}(G)$ onto itself,

then there exists $s \in \ell^{1}(G)$ such that $A^{-1}=\left[b_{i j}\right]_{i, j \in G}$ satisfies $\left|b_{i j}\right| \leq s(i-j)$ for all $i$, $j \in G$.

Most of the results described so far for irregular Gabor systems actually extend to systems of Gabor molecules, see [13, Theorem 9].

\subsection{The Definition of Localization for Gabor Systems}

In this section we give a precise definition of localization as introduced in [12, 13], but interpreted here for the particular setting of Gabor systems. Compare also the related notions introduced in [91].

Notation. The following shorthand notations will be used in this section.

(a) Given $g \in L^{2}\left(\mathbf{R}^{d}\right)$ and $\Lambda \subset \mathbf{R}^{2 d}$, we will write

$$
\mathcal{G}=\mathcal{G}(g, \Lambda)=\left\{g_{\lambda}\right\}_{\lambda \in \Lambda} .
$$

(b) Given $\phi \in L^{2}\left(\mathbf{R}^{d}\right)$ and $\alpha, \beta>0$, we will write

$$
\Delta=\alpha \mathbf{Z}^{d} \times \beta \mathbf{Z}^{d} \quad \text { and } \quad \Phi=\mathcal{G}(\phi, \Delta)=\left\{\phi_{\mu}\right\}_{\mu \in \Delta .}
$$


(c) We define $a: \Lambda \rightarrow \Delta$ to be the map such that $a(\lambda)$ is the point in $\Delta$ closest to $\lambda$ (or any map that sends a point of $\Lambda$ to a point of $\Delta$ that is within a fixed distance of the closest point). Thus the map $a$ "rounds off" to a near point of $\Delta$. Note that $a$ need not be injective.

(d) The cross-Grammian matrix of $\mathcal{G}$ and $\Phi$ is the $\Lambda \times \Delta$ matrix

$$
\left[\left\langle g_{\lambda}, \phi_{\mu}\right\rangle\right]_{\lambda \in \Lambda, \mu \in \Delta}
$$

The words "column" and "row" in the following definition refer to the cross-Grammian matrix. We think of the elements in locations $(\lambda, a(\lambda))$ as corresponding to the main diagonal of this matrix.

Definition 9 (Localization). Let $\mathcal{G}, \Phi, a$ be as above.

(a) We say that $\mathcal{G}$ is $\ell^{p}$-localized with respect to $\Phi$ and $a$, or simply that $(\mathcal{G}, a, \Phi)$ is $\ell^{p}$-localized, if

$$
\sum_{\mu \in \Delta} \sup _{\lambda \in \Lambda}\left|\left\langle g_{\lambda}, \phi_{\mu+a(\lambda)}\right\rangle\right|^{p}<\infty
$$

Equivalently, there must exist an $r \in \ell^{p}(\Delta)$ such that

$$
\forall \lambda \in \Lambda, \quad \forall \mu \in \Delta, \quad\left|\left\langle g_{\lambda}, \phi_{\mu}\right\rangle\right| \leq r_{a(\lambda)-\mu} .
$$

(b) We say that $(\mathcal{G}, a, \Phi)$ has $\ell^{p}$-column decay if

$$
\forall \varepsilon>0, \quad \exists N_{\varepsilon}>0 \quad \text { such that } \forall \mu \in \Delta, \quad \sum_{\lambda \in \Lambda \backslash Q_{N_{\varepsilon}}(\mu)}\left|\left\langle g_{\lambda}, \phi_{\mu}\right\rangle\right|^{p}<\varepsilon .
$$

(c) We say that $(\mathcal{G}, a, \Phi)$ has $\ell^{p}$-row decay if

$$
\forall \varepsilon>0, \quad \exists N_{\varepsilon}>0 \quad \text { such that } \forall \lambda \in \Lambda, \quad \sum_{\mu \in \Delta \backslash Q_{N_{\varepsilon}}(a(\lambda))}\left|\left\langle g_{\lambda}, \phi_{\mu}\right\rangle\right|^{p}<\varepsilon .
$$

(d) We say that $(\mathcal{G}, a)$ is $\ell^{p}$-self-localized if there exists an $r \in \ell^{p}(\Delta)$ such that

$$
\forall \lambda, \mu \in \Lambda, \quad\left|\left\langle g_{\lambda}, g_{\mu}\right\rangle\right| \leq r_{a(\lambda)-a(\mu)} .
$$

Thus, localization corresponds to quantifications of decay off the diagonal of the crossGrammian matrix (or of the Grammian matrix, for the case of self-localization). Obviously, many other quantifications of decay could be used, e.g., the localizations introduced by Gröchenig [91] correspond to polynomial or exponential decay off the diagonal. Also compare self-localization to the definition of intrinsic localization in [75], and the "symmetric" localization definitions in $[76,77]$.

Remark 7. (a) The fact that $\Delta$ is an additive subgroup of $\mathbf{R}^{2 d}$ and that the distance in $\mathbf{R}^{2 d}$ is invariant under shifts is essential for certain proofs in $[12,13]$.

(b) The map a given above is natural for the setting of Gabor frames, but other embeddings of $\Lambda$ into $\Delta$ could be considered.

(c) $\ell^{p}$-localization implies both $\ell^{p}$-column and $\ell^{p}$-row decay, but not conversely.

(d) If $\mathcal{G}$ is a frame, then $\ell^{2}$-column decay implies an abstraction of the Homogeneous Approximation Property (HAP) discussed in Section 5.3. In fact, it is shown in [13] that $\ell^{2}$-column decay is equivalent to the following:

$$
\forall \varepsilon>0, \quad \exists N_{\varepsilon}>0, \quad \forall \mu \in \Delta, \quad\left\|\phi_{\mu}-\sum_{\lambda \in \Lambda \cap Q_{N_{\varepsilon}}(\mu)}\left\langle\phi_{\mu}, g_{\lambda}\right\rangle \tilde{g}_{\lambda}\right\|_{2}<\varepsilon .
$$


Of course, since $\mathcal{G}$ is a frame we know that $\phi_{\mu}=\sum_{\lambda \in \Lambda}\left\langle\phi_{\mu}, g_{\lambda}\right\rangle \tilde{g}_{\lambda}$. The interest of the $\mathrm{HAP}$ is that the rate of convergence of this series does not depend on $\mu$. If $\Phi$ is also a frame, then we can deduce from this that for any $f \in L^{2}\left(\mathbf{R}^{d}\right)$, the rate of convergence of the series $M_{b} T_{a} f=\sum_{\lambda \in \Lambda}\left\langle M_{b} T_{a} f, g_{\lambda}\right\rangle \tilde{g}_{\lambda}$ does not depend on the choice of $(a, b) \in \mathbf{R}^{2 d}$. This is precisely the Strong HAP discussed in Section 5.3.

(e) $\ell^{2}$-row decay can likewise be reinterpreted as a dual HAP where the roles of $\mathcal{G}$ and $\Phi$ are interchanged. $\ell^{2}$-localization implies both $\ell^{2}$-column decay and $\ell^{2}$-row decay.

(f) It is clear that the specific form of time-frequency shifts is entering only in a very indirect way into the definition of localization. The essential features are that we have a sequence $\mathcal{G}$ which can be "compared" in some natural way to a reference sequence whose elements are indexed by an additive discrete subgroup. This suggests that we can define localization much more abstractly, and indeed, this is exactly what is done in $[91,12,13]$. The recent article $[76,77]$ introduce a modified version of the definition of localization in which the roles of $\mathcal{G}$ and $\Phi$ are more symmetric.

The localization properties of Gabor systems with respect to "nice" reference systems can be summarized as follows.

\section{Theorem 19.}

(a) If $g \in M^{p}\left(\mathbf{R}^{d}\right)$ with $1 \leq p \leq 2$ and $\phi \in M^{1}\left(\mathbf{R}^{d}\right)$, then $(\mathcal{G}, a, \Phi)$ is $\ell^{p}$-localized.

(b) If $\phi \in M^{1}\left(\mathbf{R}^{d}\right)$ and $\Phi$ is a frame for $L^{2}\left(\mathbf{R}^{d}\right)$, then we have for each $1 \leq p \leq 2$ that

$$
(\mathcal{G}, a, \Phi) \text { is } \ell^{p} \text {-localized } \Longleftrightarrow g \in M^{p}\left(\mathbf{R}^{d}\right)
$$

(c) If $g \in M^{1}\left(\mathbf{R}^{d}\right)$ then $(\mathcal{G}, a)$ is $\ell^{1}$-self-localized.

Remark 8. (a) Since $M^{2}=L^{2}$, this result implies that if $\phi \in M^{1}$, then the Gabor system $(\mathcal{G}, a, \Phi)$ is $\ell^{2}$-localized for any $g \in L^{2}\left(\mathbf{R}^{2}\right)$. In particular, $(\mathcal{G}, a, \Phi)$ has both $\ell^{2}$-column and $\ell^{2}$-row decay, and hence has both the Strong HAP and the Strong dual HAP. This is a stronger result than the Homogeneous Approximation Properties derived in [158, 98, 42], and is far more than is needed to prove the Density Theorem. And in fact, the consequences of $\ell^{2}$-localization are more than just the Density Theorem but also include the DensityMeasure Theorem and the Density-Frame Bounds Theorem as well as additional results if more localization is imposed on $g$.

(b) The key fact needed to prove Theorem 19 is that if $\phi \in M^{1}$ and $g \in M^{p}$, then the STFT $V_{\phi} g$ lies in the Wiener amalgam space $W\left(\mathcal{C}, \ell^{p}\right)$ defined in Section 2.4, see [89, Theorem 12.2.1].

(c) If we use a reference system that is not "nice" then we get weaker results. For example, if $\phi=\chi_{[0,1]^{d}}$ and $\alpha=\beta=1$ then $\Phi$ is an orthonormal basis for $L^{2}\left(\mathbf{R}^{d}\right)$ but $\phi \notin M^{1}$. While $\ell^{p}$-localization of $(\mathcal{G}, a, \Phi)$ no longer follows, it is at least still true that $(\mathcal{G}, a, \Phi)$ will have $\ell^{2}$-row decay for every $g \in L^{2}\left(\mathbf{R}^{d}\right)$; see [13, Proposition 1$]$.

It is the fact that Gabor systems possess localization properties that yields the Density Theorem and the other results for Gabor systems discussed in previous sections. In particular, the essential properties needed to prove the Density-Measure Theorem for Gabor frames (Theorem 15) are the following.

(i) Whenever $\alpha \beta<1$, we can find a "nice" reference system. That is, there exists $\phi \in M^{1}$ such that $\Phi$ is a frame for $L^{2}\left(\mathbf{R}^{d}\right)$. (Note that this does not depend 
on the Density Theorem or any other deep results - the Painless Nonorthogonal Expansions constructions are suitable examples.)

(ii) If $g \in L^{2}\left(\mathbf{R}^{d}\right)$ and $\phi \in M^{1}$, then $(\mathcal{G}, a, \Phi)$ has both $\ell^{2}$-column and $\ell^{2}$-row decay (in fact, it is $\ell^{2}$-localized, which is a stronger condition).

In the abstract setting, if the cross-Grammian matrix has sufficient decay then we will obtain an analogous Density-Measure Theorem, without reference to time-frequency shifts or other specific requirements related to representation theory or operator theory. Likewise, all the other results discussed in this section for the setting of Gabor frames have more general formulations that hold for any abstract frame which has appropriate localization. Localization, at least in the formulation of $[12,13]$, does require the existence of an appropriate reference system that is indexed by a discrete abelian group (but compare the formulations in $[91,76,77])$.

\subsection{Linear Independence of Time-Frequency Shifts}

Although not at all related to the Density Theorem (at least, not in any way that we can see), we cannot resist closing our discussion of Gabor systems by recalling our favorite open problem in Gabor theory. This is the following conjecture, which variously goes by the names of the Linear Independence Conjecture for Time-Frequency Shifts, the Zero Divisor Conjecture for the Heisenberg Group, or the HRT Conjecture.

Conjecture 2. If $g \in L^{2}(\mathbf{R})$ is nonzero and $\left\{\left(\alpha_{k}, \beta_{k}\right)\right\}_{k=1}^{N}$ is any set of finitely many distinct points in $\mathbf{R}^{2}$, then $\left\{M_{\beta_{k}} T_{\alpha_{k}} g\right\}_{k=1}^{N}$ is a linearly independent set of functions in $L^{2}(\mathbf{R})$.

Despite the striking simplicity of the statement of this conjecture, it remains open today in the generality stated. The conjecture was originally stated in [112]. Many partial or related results are known, see $[112,147,136,9]$. For example, the conjecture is known to be true if $g$ is compactly supported, if $N \leq 3$, or if $\left\{\left(\alpha_{k}, \beta_{k}\right)\right\}_{k=1}^{N}$ is a subset of a (full-rank) lattice in $\mathbf{R}^{2}$. However, even the following subconjecture is currently open.

Conjecture 3. If $g \in \mathcal{S}(\mathbf{R})$ is nonzero and $\left\{\left(\alpha_{k}, \beta_{k}\right)\right\}_{k=1}^{4}$ is any set of four distinct points in $\mathbf{R}^{2}$, then $\left\{M_{\beta_{k}} T_{\alpha_{k}} g\right\}_{k=1}^{4}$ is a linearly independent set of functions in $L^{2}(\mathbf{R})$.

For a detailed exposition of the HRT Conjecture, its context, the known partial results as of the time of writing, and related results and conjectures, see the survey [106].

\section{Wavelets}

We end this article by noting that wavelet frames behave very differently than Gabor frames with respect to density. For wavelets there is no exact analogue of the Nyquist density, even given constraints on the norm or on the admissibility constant of the wavelet (see the example of Daubechies [48, Theorem 2.10] and the more extensive results of Balan [7] and Kutyniok [140]). Thus we should not expect a full analogy of the results for localized frames in the setting of wavelets.

Even so, density conditions play a nontrivial but not-well-understood role in wavelet theory. Seip [165] introduced a notion of density for Bergman-type spaces on the unit disk, and density results for certain wavelet frames

$$
\mathcal{W}(\psi, \Lambda)=\left\{a^{-1 / 2} \psi\left(\frac{x}{a}-b\right)\right\}_{(a, b) \in \Lambda},
$$


generated by some wavelets $\psi$ follow from those results.

Additionally, a notion of affine Beurling density for irregular wavelet systems was introduced independently in [108] and [175], and various results relating frame properties of $\mathcal{W}(\psi, \Lambda)$ to the density of $\Lambda$ were obtained in those article. These definitions are not equivalent, in part because of the various choices of left versus right representation combined with left versus right translation. A comparison of these various definitions is made is [137]. In [139] it is shown that the local integrability condition of [114] is equivalent to finite affine Beurling density (assuming some mild regularity on the wavelet $\psi$ ). The role of density conditions in wave packet systems (which combine translations, modulations, and dilations together) is explored in [47].

Perhaps surprisingly, it is shown in [110] that irregular wavelet frames possess an exact analogue of the Homogeneous Approximation Property satisfied by Gabor frames (see Section 5.3). Yet, whereas in the Gabor case the HAP implies the existence of a Nyquist density, in the wavelet case the implications of the HAP are quite different. In particular, while there is a relationship between the density of any given wavelet frame compared to the density of any given wavelet Riesz basis, this relationship depends on the rate of approximation in the HAP rather than being absolute, as it is in the Gabor case.

The localized frames approach of $[12,13]$ cannot be directly applied to wavelet frames because the affine group is nonabelian and does not contain discrete subgroups. Even so, the notion of localized frames has been applied to wavelet frame expansions, see $[28,76]$. We expect that the ideas of localized frames will be an important concept for future results on wavelet frames.

\section{A. Gaussians and the Bargmann-Fock Space}

In this appendix we provide a brief summary of the relationships between properties of Gabor systems generated by the Gaussian function and properties of sets of sampling and interpolation in the Bargmann-Fock space.

First we require the following abstract terminology in addition to that given in Definition/Facts 1, cf. [189].

Definition A.1 Definition/Facts A.1. Let $\mathcal{F}=\left\{f_{n}\right\}_{n \in \mathbf{N}}$ be a sequence in a Hilbert space $H$.

(a) The analysis map or coefficient map associated to $\mathcal{F}$ is $C f=\left\{\left\langle f, f_{n}\right\rangle\right\}_{n \in \mathbf{N}}, f \in H$.

(b) The moment space of $\mathcal{F}$ is the range of $C$, which is a subspace of the space of all complex-valued sequences on $\mathbf{N}$.

(c) By the Closed Graph Theorem, $\mathcal{F}$ is a Bessel sequence if and only if range $(C) \subset \ell^{2}$.

(d) We say that $\mathcal{F}$ is a Riesz-Fischer sequence if range $(C) \supset \ell^{2}$. That is, given any $c=\left(c_{n}\right)_{n \in \mathbf{N}}$, there exists $f \in H$ such that $\left\langle f, f_{n}\right\rangle=c_{n}$ for all $n \in \mathbf{N}$.

(e) $\mathcal{F}$ is a Riesz sequence if and only if $\mathcal{F}$ is both a Bessel sequence and a Riesz-Fischer sequence.

Now we turn to the Bargmann transform and the Bargmann-Fock space. We will only consider the case $d=1$ and $p=2$. We refer to [73, Section 1.6] and [89, Section 3.4] for details. Throughout, $\varphi(t)=2^{1 / 4} e^{-\pi t^{2}}$ will denote the one-dimensional normalized Gaussian window function. 


\section{Definition A.2 Definition/Facts A.2.}

(a) The (Bargmann-)Fock space $\mathcal{F}^{2}(\mathbf{C})$ consists of all entire functions $F$ on $\mathbf{C}$ for which

$$
\|F\|_{\mathcal{F}}^{2}=\int_{\mathbf{C}}|F(z)|^{2} e^{-\pi|z|^{2}} d z<\infty .
$$

$\mathcal{F}^{2}(\mathbf{C})$ is a Hilbert space under the inner product

$$
\langle F, G\rangle_{\mathcal{F}}=\int_{\mathbf{C}} F(z) \overline{G(z)} e^{-\pi|z|^{2}} d z .
$$

(b) The Bargmann transform of a function $f$ on $\mathbf{R}$ is the function $B f$ on $\mathbf{C}$ given by

$$
B f(z)=2^{1 / 4} \int_{\mathbf{R}} f(t) e^{2 \pi i t z-\pi t^{2}-\frac{\pi}{2} z^{2}} d t
$$

whenever this is defined.

(c) The Bargmann transform $B$ is a unitary map of $L^{2}(\mathbf{R})$ onto $\mathcal{F}^{2}(\mathbf{C})$.

(d) If we write $z=x+i \xi$, then

$$
V_{\varphi} f(x,-\xi)=e^{\pi i x \xi} B f(z) e^{-\frac{\pi}{2}|z|^{2}} .
$$

(e) If $F \in \mathcal{F}^{2}(\mathbf{C})$, then for $z=x+i \xi \in \mathbf{C}$ we have

$$
\left\langle F, B\left(T_{x} M_{-\xi} \varphi\right)\right\rangle_{\mathcal{F}}=e^{-\pi i x \xi} e^{-\frac{\pi}{2}|z|^{2}} F(z) .
$$

Next we define sets of sampling and interpolation, cf. [164, 168].

Definition A.3. Let $\Gamma=\left\{z_{n}\right\}_{n \in \mathbf{N}}$ be a sequence of points in $\mathbf{C}$.

(a) $\Gamma$ is a set of sampling for $\mathcal{F}^{2}(\mathbf{C})$ if there exist $A, B>0$ such that

$$
\forall F \in \mathcal{F}^{2}(\mathbf{C}), \quad A\|F\|_{\mathcal{F}}^{2} \leq \sum_{n=1}^{\infty} e^{-\pi\left|z_{n}\right|^{2}}\left|F\left(z_{n}\right)\right|^{2} \leq B\|F\|_{\mathcal{F}}^{2} .
$$

(b) $\Gamma$ is a set of interpolation for $\mathcal{F}^{2}(\mathbf{C})$ if for every $c=\left(c_{n}\right)_{n \in \mathbf{N}} \in \ell^{2}$ there exists $F \in \mathcal{F}^{2}(\mathbf{C})$ such that

$$
e^{-\frac{\pi}{2}\left|z_{n}\right|^{2}} F\left(z_{n}\right)=c_{n}, \quad n \in \mathbf{N} .
$$

Now we can derive the equivalences between sets of sampling and interpolation and frame properties of Gabor systems generated by the Gaussian window. The Beurling density of a sequence of complex numbers is defined via the natural identification of $\mathbf{C}$ with $\mathbf{R}^{2}$.

Theorem A.1. Let $\Gamma=\left\{z_{n}\right\}_{n \in \mathbf{N}}$ be a sequence of points in $\mathbf{C}$ such that $D^{+}(\Gamma)<\infty$ (equivalently, $\Gamma$ is a union of finitely many uniformly separated subsequences). Define

$$
\Lambda=\left\{\left(x_{n},-\xi_{n}\right): z_{n}=x_{n}+i \xi_{n}, n \in \mathbf{N}\right\} .
$$

Then the following statements hold.

(a) $\Gamma$ is a set of sampling for $\mathcal{F}^{2}(\mathbf{C})$ if and only if $\mathcal{G}(g, \Lambda)$ is a frame for $L^{2}(\mathbf{R})$. 
(b) $\Gamma$ is a set of interpolation for $\mathcal{F}^{2}(\mathbf{C})$ if and only if $\mathcal{G}(g, \Lambda)$ is a Riesz sequence in $L^{2}(\mathbf{R})$.

Proof. (a) Suppose that $\Gamma$ is a set of sampling. If $f \in L^{2}(\mathbf{R})$ and $F=B f$, then $\|f\|_{2}=\|F\|_{\mathcal{F}}$ and

$$
\sum_{n=1}^{\infty} e^{-\pi\left|z_{n}\right|^{2}}\left|F\left(z_{n}\right)\right|^{2}=\sum_{n=1}^{\infty}\left|\left\langle F, B\left(T_{x_{n}} M_{-\xi_{n}} \varphi\right)\right\rangle_{\mathcal{F}}\right|^{2}=\sum_{n=1}^{\infty}\left|\left\langle f, T_{x_{n}} M_{-\xi_{n}} \varphi\right\rangle\right|^{2} .
$$

Therefore $\mathcal{G}(\varphi, \Lambda)$ is a frame for $L^{2}(\mathbf{R})$, and the converse is symmetric.

(b) Suppose that $\Gamma$ is a set of interpolation and let $c=\left(c_{n}\right)_{n \in \mathbf{N}} \in \ell^{2}$ be given. Then $\left(e^{\pi i x_{n} \xi_{n}} c_{n}\right)_{n \in \mathbf{N}} \in \ell^{2}$, so there exists $F \in \mathcal{F}^{2}(\mathbf{C})$ such that

$$
e^{-\frac{\pi}{2}\left|z_{n}\right|^{2}} F\left(z_{n}\right)=e^{\pi i x_{n} \xi_{n}} c_{n}, \quad n \in \mathbf{N} .
$$

Setting $f=B^{-1} F \in L^{2}(\mathbf{R})$, we therefore have that

$$
\left\langle f, T_{x_{n}} M_{-\xi_{n}} \varphi\right\rangle=\left\langle F, B\left(T_{x_{n}} M_{-\xi_{n}} \varphi\right)\right\rangle_{\mathcal{F}}=e^{-\pi i x_{n} \xi_{n}} e^{-\frac{\pi}{2}\left|z_{n}\right|^{2}} F\left(z_{n}\right)=c_{n} .
$$

Thus $\mathcal{G}(\varphi, \Lambda)$ is a Riesz-Fischer sequence in $L^{2}(\mathbf{R})$. However, since $\varphi \in M^{1}(\mathbf{R})$ and we have assumed that $D^{+}(\Lambda)=D^{+}(\Gamma)<\infty$, we have by Theorem 12 that $\mathcal{G}(\varphi, \Lambda)$ is a Bessel sequence, and hence by Definition/Facts A.1(e), it must be a Riesz sequence in $L^{2}(\mathbf{R})$. The converse is symmetric.

Actually, with $\Gamma$ and $\Lambda$ related as in (A.1), any of the hypotheses that $\Gamma$ is a set of sampling, $\mathcal{G}(g, \Lambda)$ is a frame, or $\mathcal{G}(g, \Lambda)$ is a Riesz sequence imply that $D^{+}(\Gamma)<\infty$. However, the hypothesis that $\Gamma$ is a set of interpolation does not by itself imply finite density.

Combining the Density Theorem (Theorem 13) and Theorem A.1, we see that if $\Gamma$ is a set of sampling then we must have $1 \leq D^{+}(\Gamma)<\infty$, and if $\Gamma$ is a set of interpolation with finite density then $0 \leq D^{+}(\Gamma) \leq 1$. Lyubarskii and Seip/Wallstén proved more. The following result summarizes Theorems 2.1, 2.2 in [164] and Theorems 1.1, 1.2 in [168].

Theorem A.2. Let $\Gamma$ be a sequence of points in $\mathbf{C}$.

(a) $\Gamma$ is a set of sampling for $\mathcal{F}^{2}(\mathbf{C})$ if and only if $\Gamma$ is a union of finitely many uniformly separated sets $\Gamma_{1}, \ldots, \Gamma_{N}$, and $\Gamma$ contains a uniformly separated set $\Gamma^{\prime}$ such that $D^{-}(\Gamma)>1$.

(b) $\Gamma$ is a set of interpolation for $\mathcal{F}^{2}(\mathbf{C})$ with $D^{+}(\Gamma)<\infty$ if and only if $\Gamma$ is uniformly separated and $D^{+}(\Gamma)<1$.

Using the equivalences obtained above, we can translate Theorem A.1 into necessary and sufficient conditions for $\mathcal{G}(\varphi, \Lambda)$ to be a frame or a Riesz sequence. When we do this, we obtain Theorem 14.

\section{B. Ultrafilters}

In this appendix we provide a brief review of ultrafilters and their basic properties. For additional information, we refer to [115, Chapter 3]. Filters were introduced by H. Cartan $[29,30]$ in order to characterize continuous functions on general topological spaces. Soon after, it was realized that the set of ultrafilters endowed with the proper topology is the 
Stone-Čech compactification of a discrete (or more generally, a completely regular) topological space. In the following we will restrict our attention to ultrafilters over the natural numbers $\mathbf{N}$.

Definition B.1. A collection $p$ of subsets of $\mathbf{N}$ is a filter if:

(a) $\emptyset \notin p$,

(b) if $A, B \in p$ then $A \cap B \in p$,

(c) if $A \in p$ and $A \subset B \subset \mathbf{N}$, then $B \in p$.

A filter $p$ is an ultrafilter if it is maximal in the sense that:

(d) if $p^{\prime}$ is a filter on $\mathbf{N}$ such that $p \subset p^{\prime}$, then $p^{\prime}=p$,

or, equivalently, if

(d') for any $A \subset \mathbf{N}$, either $A \in p$ or $\mathbf{N} \backslash A \in p$ (but not both, because of properties a and $\mathrm{b}$ ).

The set of ultrafilters is denoted by $\beta \mathbf{N}$.

Definition B.2. Given any $n \in \mathbf{N}, e_{n}=\{A \subset \mathbf{N}: n \in A\}$ is an ultrafilter, called a principal ultrafilter. It is straightforward to show that any ultrafilter $p$ that contains a finite set must be one of these principal ultrafilters. An ultrafilter which contains no finite sets is called free. The set of free ultrafilters is denoted by $\mathbf{N}^{*}$.

Our main use for ultrafilters is that they provide a notion of convergence for arbitrary sequences.

Definition B.3. Let $p \in \beta \mathbf{N}$ be an ultrafilter. Then we say that a sequence $\left\{c_{k}\right\}_{k \in \mathbf{N}}$ of complex numbers converges to $c \in \mathbf{C}$ with respect to $p$ if for every $\varepsilon>0$ there exists a set $A \in p$ such that $\left|c_{k}-c\right|<\varepsilon$ for all $k \in A$. In this case we write $c=p$ - $\lim _{k \in \mathbf{N}} c_{k}$ or simply $c=p$-lim $c_{k}$.

The following proposition summarizes the basic properties of convergence with respect to an ultrafilter.

Proposition B.1. Let $p \in \beta \mathbf{N}$ be an ultrafilter. Then the following statements hold.

(a) Every bounded sequence of complex scalars $\left\{c_{k}\right\}_{k \in \mathbf{N}}$ converges with respect to $p$ to some $c \in \mathbf{C}$.

(b) p-limits are unique.

(c) If $p=e_{n}$ is a principal ultrafilter, then $p-\lim c_{k}=c_{n}$.

(d) If $\left\{c_{k}\right\}_{k \in \mathbf{N}}$ is a convergent sequence in the usual sense, $p$ is a free ultrafilter, and $\lim _{k \rightarrow \infty} c_{k}=c$, then $p-\lim c_{k}=c$.

(e) If $\left\{c_{k}\right\}_{k \in \mathbf{N}}$ is a bounded sequence and $p$ is a free ultrafilter, then $p$ - $\lim _{k \in \mathbf{N}} c_{k}$ is an accumulation point of $\left\{c_{k}\right\}_{k \in \mathbf{N}}$.

(f) If $c$ is an accumulation point of a bounded sequence $\left\{c_{k}\right\}_{k \in \mathbf{N}}$, then there exists a free ultrafilter $p$ such that $p$ - $\lim c_{k}=c$. In particular, there exists an ultrafilter $p$ such that $p-\lim c_{k}=\lim \sup c_{k}$, and there exists an ultrafilter $q$ such that $q-\lim c_{k}=$ $\liminf c_{k}$.

(g) $p$-limits are linear, i.e., $p$ - $\lim \left(a c_{k}+b d_{k}\right)=a p$ - $\lim c_{k}+b p$-lim $d_{k}$.

(h) $p$-limits respect products, i.e., $p$ - $\lim \left(c_{k} d_{k}\right)=\left(p-\lim c_{k}\right)\left(p\right.$-lim $\left.d_{k}\right)$. 


\section{Acknowledgments}

We thank Hans Feichtinger, Fumiko Futamura, Deguang Han, Gitta Kutyniok, and Götz Pfander for helpful comments and suggestions of references. Guido Janssen provided a variety of comments on various versions of this manuscript, for which we are extremely grateful. We are especially indebted to Karlheinz Gröchenig, both for personal discussions and for his invaluable text [89], which has strongly influenced the writing of this article. Of course, all inaccuracies or omissions in this article are solely the fault of the author.

\section{References}

[1] A. Aldroubi and K. Gröchenig (2001). Nonuniform sampling and reconstruction in shiftinvariant spaces, SIAM Rev. 43, 585-620.

[2] P. Auscher (1994). Remarks on the local Fourier bases, in Wavelets: Mathematics and Applications, J. J. Benedetto and M. W. Frazier, Eds., CRC Press, Boca Raton, FL, 203-218.

[3] P. Auscher, G. Weiss, and M. V. Wickerhauser (1992). Local sine and cosine basis of Coifman and Meyer and the construction of smooth wavelets, in Wavelets: A Tutorial in Theory and Applications, C. K. Chui, Ed., Academic Press, Boston, 273-256.

[4] L. Auslander and R. Tolimieri (1985). Radar ambiguity functions and group theory, SIAM J. Math. Anal. 16, 577-601.

[5] H. Bacry, A. Grossmann, and J. Zak (1975). Proof of completeness of lattice states in the kq representation, Phys. Rev. B 12, 1118-1120.

[6] L. Baggett (1990). Processing a radar signal and representations of the discrete Heisenberg group, Colloq. Math. 60/61, 195-203.

[7] R. Balan (1997). Stability theorems for Fourier frames and wavelet Riesz bases, J. Fourier Anal. Appl. 3, 499-504.

[8] R. Balan (1998), Extensions of no-go theorems to many signal systems, in Wavelets, Multiwavelets, and their Applications (San Diego, CA, 1997), Contemp. Math., Vol. 216, A. Aldroubi and E.-B. Lin, Eds., Amer. Math. Soc., Providence, RI, 3-14.

[9] R. Balan (2006). The noncommutative Wiener lemma, linear independence, and spectral properties of the algebra of time-frequency shift operators, Trans. Amer. Math. Soc., to appear.

[10] R. Balan, P. G. Casazza, C. Heil, and Z. Landau (2003). Deficits and excesses of frames, Adv. Comput. Math. 18, 93-116.

[11] R. Balan, P. G. Casazza, C. Heil, and Z. Landau (2003). Excesses of Gabor frames, Appl. Comput. Harmon. Anal. 14, 87-106.

[12] R. Balan, P. G. Casazza, C. Heil, and Z. Landau (2006). Density, overcompleteness, and localization of frames, I. Theory, J. Fourier Anal. Appl. 12, 105-143.

[13] R. Balan, P. G. Casazza, C. Heil, and Z. Landau (2006) Density, overcompleteness, and localization of frames, II. Gabor frames, J. Fourier Anal. Appl. 12, 307-344.

[14] R. Balian (1981). Un principe d'incertitude fort en théorie du signal ou en mécanique quantique, C. R. Acad. Sci. Paris 292, 1357-1362.

[15] V. Bargmann, P. Butera, L. Girardello, and J. R. Klauder (1971). On the completeness of coherent states, Rep. Math. Phys. 2, 221-228.

[16] A. G. Baskakov (1990). Wiener's theorem and asymptotic estimates for elements of inverse matrices, Funktsional. Anal. i Prilozhen. 24, pp. 64-65; translation in Funct. Anal. Appl. 24, pp. 222-224.

[17] G. Battle (1988). Heisenberg proof of the Balian-Low theorem, Lett. Math. Phys. 15, 175-177.

[18] B. Bekka (2004). Square integrable representations, von Neumann algebras and an application to Gabor analysis, J. Fourier Anal. Appl. 10, 325-349.

[19] J. J. Benedetto, W. Czaja, and A. Ya. Maltsev (2003). The Balian-Low theorem for the symplectic form on $\mathbf{R}^{2 d}$, J. Math. Phys. 44, 1735-1750.

[20] J. J. Benedetto, W. Czaja, P. Gadziński, and A. M. Powell (2003). The Balian-Low Theorem 
and regularity of Gabor systems, J. Geom. Anal. 13, 239-254.

[21] J. J. Benedetto, W. Czaja, and A. M. Powell (2006). An optimal example for the Balian-Low uncertainty principle, SIAM J. Math. Anal. 38, 333-345.

[22] J. J. Benedetto, W. Czaja, A. M. Powell, and J. Sterbenz (2006). An endpoint $(1, \infty)$ BalianLow theorem, Math. Res. Lett. 13, 467-474.

[23] J. J. Benedetto, C. Heil, and D. F. Walnut (1995). Differentiation and the Balian-Low theorem, J. Fourier Anal. Appl. 1, 355-402.

[24] J. J. Benedetto, A. Powell, and O. Yilmaz (2006). Sigma-Delta quantization and finite frames, IEEE Trans. Inform. Theory 52, 1990-2005.

[25] K. Bittner and C. K. Chui (2002). Gabor frames with arbitrary windows, in Approximation Theory, X (St. Louis, MO, 2001), C. K. Chui, L. L. Schumaker, and J. Stöckler, Eds., Vanderbilt Univ. Press, Nashville, TN, 41-50,

[26] H. Bölcskei and A. J. E. M. Janssen (2000). Gabor frames, unimodularity, and window decay, J. Fourier Anal. Appl. 6, 255-276.

[27] M. Bownik and Z. Rzeszotnik (2003). The spectral function of shift-invariant spaces, Michigan Math. J. 51, 387-414

[28] M. Bownik and D. Speegle (2006). The Feichtinger conjecture for wavelet frames, Gabor frames and frames of translates, Canad. J. Math. 58, 1121-1143.

[29] H. Cartan (1937). Théorie des filtres, C. R. Acad. Sci. Paris 205, 595-598.

[30] H. Cartan (1937). Filtres et ultrafiltres, C. R. Acad. Sci. Paris 205, 777-779.

[31] P. G. Casazza and O. Christensen (1998). Frames containing a Riesz basis and preservation of this property under perturbations, SIAM J. Math. Anal. 29, 266-278.

[32] P. G. Casazza and O. Christensen (1998). Frames and Schauder bases, in Approximation Theory, N. K. Govil et al., Eds., Dekker, New York, 133-139.

[33] P. G. Casazza, O. Christensen, A. M. Lindner, and R. Vershynin (2005). Frames and the Feichtinger conjecture, Proc. Amer. Math. Soc. 133, 1025-1033.

[34] P. G. Casazza, O. Christensen, and A. J. E. M. Janssen (2001). Weyl-Heisenberg frames, translation invariant systems and the Walnut representation, J. Funct. Anal. 180, 85-147.

[35] P. G. Casazza, O. Christensen, and M. C. Lammers (2002). Perturbations of Weyl-Heisenberg frames, Hokkaido Math. J. 31, 539-553.

[36] P. G. Casazza, D. Han, and D. R. Larson (1999). Frames for Banach spaces, in The Functional and Harmonic Analysis of Wavelets and Frames (San Antonio, TX, 1999), Contemp. Math., Vol. 247, L. W. Baggett and D. R. Larson, Eds., Amer. Math. Soc., Providence, RI, 149-182.

[37] P. G. Casazza and N. J. Kalton (2002). Roots of complex polynomials and Weyl-Heisenberg frame sets, Proc. Amer. Math. Soc. 130, 2313-2318.

[38] P. G. Casazza, G. Kutyniok, and M. C. Lammers (2004). Duality principles in frame theory, J. Fourier Anal. Appl. 10, 383-408.

[39] P. G. Casazza and J. C. Tremain (2006). The Kadison-Singer problem in mathematics and engineering, Proc. Natl. Acad. Sci. USA 103, 2032-2039.

[40] O. Christensen (1998). Perturbation of frames and applications to Gabor frames, in Gabor Analysis and Algorithms: Theory and Applications, H. G. Feichtinger and T. Strohmer, Eds., Birkhäuser, Boston, 193-209.

[41] O. Christensen (2003). An Introduction to Frames and Riesz Bases, Birkhäuser, Boston.

[42] O. Christensen, B. Deng, and C. Heil (1999). Density of Gabor frames, Appl. Comput. Harmon. Anal. 7, 292-304.

[43] O. Christensen, S. Favier, and Z. Felipe (2001). Irregular wavelet frames and Gabor frames, Approx. Theory Appl. (N.S.) 17, 90-101.

[44] O. Christensen and C. Heil (1997). Perturbations of Banach frames and atomic decompositions, Math. Nachr. 185, 33-47.

[45] R. R. Coifman and Y. Meyer (1991). Remarques sur l'analyse de Fourier à fenêtre, C. R. Acad. Sci. Paris Sér. I Math., 312, 259-261.

[46] E. Cordero and K. Gröchenig (2004). Localization of frames. II, Appl. Comput. Harmon. Anal. 17, 29-47.

[47] W. Czaja, G. Kutyniok, and D. Speegle (2006). The geometry of sets of parameters of wave 
packet frames, Appl. Comput. Harmon. Anal. 20, 108-125.

[48] I. Daubechies (1990). The wavelet transform, time-frequency localization and signal analysis, IEEE Trans. Inform. Theory 39, 961-1005.

[49] I. Daubechies (1992). Ten Lectures on Wavelets, SIAM, Philadelphia.

[50] I. Daubechies and A. Grossmann (1988). Frames in the Bargmann space of entire functions, Comm. Pure Appl. Math. 41, 151-164.

[51] I. Daubechies, A. Grossmann, and Y. Meyer (1986). Painless nonorthogonal expansions, J. Math. Phys. 27, 1271-1283.

[52] I. Daubechies, S. Jaffard, and J.-L. Journé (1991). A simple Wilson orthonormal basis with exponential decay, SIAM J. Math. Anal. 22, 554-573.

[53] I. Daubechies and A. J. E. M. Janssen (1993). Two theorems on lattice expansions, IEEE Trans. Inform. Theory 39, 3-6.

[54] I. Daubechies, H. Landau, and Z. Landau (1995). Gabor time-frequency lattices and the Wexler-Raz identity, J. Fourier Anal. Appl. 1, 437-478.

[55] B. Deng and C. Heil (2000). Density of Gabor Schauder bases, in Wavelet Applications in Signal and Image Processing VIII (San Diego, CA, 2000), A. Aldroubi at al., Eds., Proc. SPIE Vol. 4119, SPIE, Bellingham, WA, 153-164.

[56] M. Dörfler (2001). Time-frequency analysis for music signals: A mathematical approach, J. New Music Research 30, 3-12.

[57] R. J. Duffin and A. C. Schaeffer (1952). A class of nonharmonic Fourier series, Trans. Amer. Math. Soc. 72, 341-366.

[58] Y. C. Eldar and G. D. Forney (2002). Optimal tight frames and quantum measurement, IEEE Trans. Information Theory 48, 599-610.

[59] S. J. Favier and R. A. Zalik (1995). On the stability of frames and Riesz bases, Appl. Comput. Harmon. Anal. 2, 160-173.

[60] H. G. Feichtinger (1980). Banach convolution algebras of Wiener type, in Functions, Series, Operators, Proc. Conf. Budapest 38, Colloq. Math. Soc. János Bolyai, 509-524.

[61] H. G. Feichtinger (1981). On a new Segal algebra, Monatsh. Math. 92, 269-289.

[62] H. G. Feichtinger (1989). Atomic characterizations of modulation spaces through Gabor-type representations, Rocky Mountain J. Math. 19, 113-125.

[63] H. G. Feichtinger (2006). Modulation spaces: Looking back and ahead, Sampl. Theory Signal Image Process. 5, 109-140.

[64] H. G. Feichtinger and K. Gröchenig (1989). Banach spaces related to integrable group representations and their atomic decompositions, I, J. Funct. Anal. 86, 307-340.

[65] H. G. Feichtinger and K. Gröchenig (1989). Banach spaces related to integrable group representations and their atomic decompositions, II, Monatsh. Math. 108, 129-148.

[66] H. G. Feichtinger and K. Gröchenig (1992). Gabor wavelets and the Heisenberg group: Gabor expansions and short-time Fourier expansion from the group theoretic point of view, in Wavelets: A Tutorial in Theory and Applications, C. K. Chui, Ed., Academic Press, Boston, 359-397.

[67] H. G. Feichtinger and K. Gröchenig (1997). Gabor frames and time-frequency analysis of distributions, J. Funct. Anal. 146, 464-495.

[68] H. G. Feichtinger, K. Gröchenig, and D. Walnut (1992). Wilson bases and modulation spaces, Math. Nachr. 155, 7-17.

[69] H. G. Feichtinger and A. J. E. M. Janssen (2000). Validity of WH-frame bound conditions depends on lattice parameters, Appl. Comput. Harmon. Anal. 8, 104-112.

[70] H. G. Feichtinger and N. Kaiblinger (2004). Varying the time-frequency lattice of Gabor frames, Trans. Amer. Math. Soc. 356, 2001-2023.

[71] H. G. Feichtinger and W. Kozek (1998). Quantization of TF lattice-invariant operators on elementary LCA groups, in Gabor Analysis and Algorithms: Theory and Applications, H. G. Feichtinger and T. Strohmer, Eds., Birkhäuser, Boston, 233-266.

[72] H. G. Feichtinger and G. Zimmermann (1998). A Banach space of test functions for Gabor analysis, in Gabor Analysis and Algorithms: Theory and Applications, H. G. Feichtinger and T. Strohmer, Eds., Birkhäuser, Boston, 123-170. 
[73] G. B. Folland (1989). Harmonic Analysis on Phase Space, Princeton University Press, Princeton, NJ.

[74] G. B. Folland (2006). The abstruse meets the applicable: Some aspects of time-frequency analysis, Proc. Indian Acad. Sci. Math. Sci. 116, 121-136.

[75] M. Fornasier and K. Gröchenig (2005). Intrinsic localization of frames, Constr. Approx. 22, 395-415.

[76] F. Futamura (2006). Banach framed, decay in the context of localization, Sampl. Theory Signal Image Process., to appear.

[77] F. Futamura (2007). Symmetrically localized frames and the removal of subsets of positive density, J. Math. Anal. Appl. 326, 1225-1235.

[78] J.-P. Gabardo and D. Han (2003). Frame representations for group-like unitary operator systems, J. Operator Theory 49, 223-244.

[79] J.-P. Gabardo and D. Han (2004). Balian-Low phenomenon for subspace Gabor frames, J. Math. Phys. 45, 3362-3378.

[80] D. Gabor (1946). Theory of communications, J. Inst. Elec. Eng. (London) 93, 429-457.

[81] I. M. Gel'fand (1950). Expansion in characteristic functions of an equation with periodic coefficients (Russian), Doklady Akad. Nauk SSSR (N.S.) 73, 1117-1120.

[82] I. Gohberg, M. A. Kaashoek, and H. J. Woerdeman (1989). The band method for positive and contractive extension problems, J. Operator Theory 22, 109-155.

[83] V. K. Goyal, J. Kovačević, and J. A. Kelner (2001). Quantized frame expansions with erasures, Appl. Comput. Harmon. Anal. 10, 203-233.

[84] R. Gribonval and M. Nielsen (2004). Nonlinear approximation with dictionaries. I. Direct estimates, J. Fourier Anal. Appl. 10, 51-71.

[85] R. Gribonval and M. Nielsen (2004). On a problem of Gröchenig about nonlinear approximation with localized frames, J. Fourier Anal. Appl. 10, 433-437.

[86] K. Gröchenig (1991). Describing functions: Atomic decompositions versus frames, Monatsh. Math. 112, 1-42.

[87] K. Gröchenig (1993). Irregular sampling of wavelet and short-time Fourier transforms, Constr. Approx. 9, 283-297.

[88] K. Gröchenig (1998), Aspects of Gabor analysis on locally compact abelian groups, in Gabor Analysis and Algorithms: Theory and Applications, H. G. Feichtinger and T. Strohmer, Eds., Birkhäuser, Boston, 211-231.

[89] K. Gröchenig (2001). Foundations of Time-Frequency Analysis, Birkhäuser, Boston.

[90] K. Gröchenig (2003). Localized frames are finite unions of Riesz sequences, Adv. Comput. Math. 18, 149-157.

[91] K. Gröchenig (2004). Localization of frames, Banach frames, and the invertibility of the frame operator, J. Fourier Anal. Appl. 10, 105-132.

[92] K. Gröchenig (2006). Time-frequency analysis of Sjöstrand's class, Rev. Mat. Iberoamericana 22, 703-724.

[93] K. Gröchenig, D. Han, C. Heil, and G. Kutyniok (2002). The Balian-Low Theorem for symplectic lattices in higher dimensions, Appl. Comput. Harmon. Anal. 13, 169-176.

[94] K. Gröchenig and C. Heil (1999). Modulation spaces and pseudodifferential operators, Integral Equations Operator Theory 34, 439-457.

[95] K. Gröchenig and C. Heil (2001). Gabor meets Littlewood-Paley: Gabor expansions in $L^{p}\left(\mathbf{R}^{d}\right)$, Studia Math. 146, 15-33.

[96] K. Gröchenig, C. Heil, and K. Okoudjou (2002). Gabor analysis in weighted amalgam spaces, Sampl. Theory Signal Image Process. 1, 225-259.

[97] K. Gröchenig and M. Leinert (2004). Wiener's lemma for twisted convolution and Gabor frames, J. Amer. Math. Soc. 17, 1-18.

[98] K. Gröchenig and H. Razafinjatovo (1996). On Landau's necessary density conditions for sampling and interpolation of band-limited functions, J. London Math. Soc. (2) 54, 557-565.

[99] K. Gröchenig and T. Strohmer (2006). Pseudodifferential operators on locally compact abelian groups and Sjöstrand's symbol class, J. Reine Angew. Math., to appear.

[100] D. Han and D. R. Larson (2000). Frames, bases and group representations Mem. Amer. Math. 
Soc. 147(697).

[101] D. Han and Y. Wang (2001). Lattice tiling and the Weyl-Heisenberg frames, Geom. Funct. Anal. 11, 742-758.

[102] D. Han and Y. Wang (2004). The existence of Gabor bases and frames, in Wavelets, Frames and Operator Theory, C. Heil, D. R. Larson, and P. E. T. Jorgensen, Eds., Contemp. Math., Vol. 345, Amer. Math. Soc., Providence, RI, 183-192.

[103] C. Heil (1990). Wiener amalgam spaces in generalized harmonic analysis and wavelet theory, Ph.D. Thesis, University of Maryland, College Park, MD.

[104] C. Heil (2003). Integral operators, pseudodifferential operators, and Gabor frames, in Advances in Gabor Analysis, H. G. Feichtinger and T. Strohmer, Eds., Birkhäuser, Boston, 153-169.

[105] C. Heil (2003). An introduction to weighted Wiener amalgams, in Wavelets and their Applications (Chennai, January 2002), M. Krishna, R. Radha and S. Thangavelu, Eds., Allied Publishers, New Delhi, 183-216.

[106] C. Heil (2006). Linear independence of finite Gabor systems, in: "Harmonic Analysis and Applications," C. Heil, Ed., Birkhäuser, Boston, 171-206.

[107] C. Heil (2007). The Density Theorem and the Homogeneous Approximation Property for Gabor frames, in Representations, Wavelets, and Frames, P. E. T. Jorgensen, K. D. Merrill, and J. D. Packer, Eds., Birkhäuser, Boston, to appear.

[108] C. Heil and G. Kutyniok (2003). Density of weighted wavelet frames, J. Geom. Anal. 13, 479-493.

[109] C. Heil and G. Kutyniok (2006). Density of frames and Schauder bases of windowed exponentials, Houston J. Math., to appear.

[110] C. Heil and G. Kutyniok (2006). The Homogeneous Approximation Property for wavelet frames, J. Approx. Theory, to appear.

[111] C. Heil and A. M. Powell (20006). Gabor Schauder bases and the Balian-Low Theorem, J. Math. Physics, 47, 113506-1-113506-21.

[112] C. Heil, J. Ramanathan, and P. Topiwala (1996). Linear independence of time-frequency translates, Proc. Amer. Math. Soc. 124, 2787-2795.

[113] C. E. Heil and D. F. Walnut (1989). Continuous and discrete wavelet transforms, SIAM Review 31, 628-666.

[114] E. Hernández, D. Labate, and G. Weiss (2002). A unified characterization of reproducing systems generated by a finite family, II, J. Geom. Anal. 12, 615-662.

[115] N. Hindman and D. Strauss (1998). Algebra in the Stone-Čech Compactification, de Gruyter Expositions in Mathematics 27, Walter de Gruyter and Co., Berlin.

[116] J.R. Holub (1994). Pre-frame operators, Besselian frames, and near-Riesz bases in Hilbert spaces, Proc. Amer. Math. Soc. 122, 779-785.

[117] S. Jaffard (1990). Propriétés des matrices "bien localisées" près de leur diagonale et quelques applications, Ann. Inst. H. Poincaré Anal. Non Linéaire, 7, pp. 461-476.

[118] A. J. E. M. Janssen (1981). Gabor representation of generalized functions, J. Math. Anal. Appl. 83, 377-394.

[119] A. J. E. M. Janssen (1982). Bargmann transform, Zak transform, and coherent states, J. Math. Phys. 23, 720-731.

[120] A. J. E. M. Janssen (1988). The Zak transform: a signal transform for sampled time-continuous signals, Philips J. Res. 43, 23-69.

[121] A. J. E. M. Janssen (1994). Signal analytic proofs of two basic results on lattice expansions, Appl. Comput. Harmon. Anal. 1, 350-354.

[122] A. J. E. M. Janssen (1995). Duality and biorthogonality for Weyl-Heisenberg frames, J. Fourier Anal. Appl. 1, 403-436.

[123] A. J. E. M. Janssen (1995). On rationally oversampling Weyl-Heisenberg frames, Signal Proc. 47, 239-245.

[124] A. J. E. M. Janssen (1996). Some counterexamples in the theory of Weyl-Heisenberg frames, IEEE Trans. Inform. Theory 42, 621-623.

[125] A. J. E. M. Janssen (1998). The duality condition for Weyl-Heisenberg frames, in Gabor Analysis and Algorithms: Theory and Applications, H. G. Feichtinger and T. Strohmer, Eds., 
Birkhäuser, Boston, 33-84.

[126] A. J. E. M. Janssen (1998). A density theorem for time-continuous filter banks, in Signal and Image Representation in Combined Spaces, Y. Y. Zeevi and R. R. Coifman, Eds., Wavelet Anal. Appl., Vol. 7, Academic Press, San Diego, CA, 513-523.

[127] A. J. E. M. Janssen (2001). Representations of Gabor frame operators, in Twentieth century harmonic analysis - a celebration (Il Ciocco, 2000), NATO Sci. Ser. II Math. Phys. Chem., 33, Kluwer Acad. Publ., Dordrecht, 73-101.

[128] A. J. E. M. Janssen (2003). Zak transforms with few zeros and the tie, in Advances in Gabor Analysis, H. G. Feichtinger and T. Strohmer, Eds., Birkhäuser, Boston, 31-70.

[129] A. J. E. M. Janssen (2003). On generating tight Gabor frames at critical density, J. Fourier Anal. Appl. 9, 175-214.

[130] A. J. E. M. Janssen (2005). Classroom proof of the Density Theorem for Gabor systems, ESI preprint \#1649; available electronically, http://www.esi.ac.at/preprints/ESI-Preprints.html.

[131] A. J. E. M. Janssen and T. Strohmer (2002). Hyperbolic secants yield Gabor frames, Appl. Comput. Harmon. Anal. 12, 259-267.

[132] E. Kaniuth and G. Kutyniok (1999). Zeros of the Zak transform on locally compact abelian groups, Proc. Amer. Math. Soc. 126, 3561-3569.

[133] K. S. Kazarian (1976). The multiplicative completion of basic sequences in $L^{p}, 1 \leq p<\infty$, to bases in $L^{p}$ (Russian), Akad. Nauk Armjan. SSR Dokl. 62, 203-209.

[134] K. S. Kazarian (1978). The multiplicative complementation of some incomplete orthonormal systems to bases in $L^{p}, 1 \leq p<\infty$ (Russian), Anal. Math. 4, 37-52.

[135] W. Kozek (1998). Adaptation of Weyl-Heisenberg frames to underspread environments, in Gabor Analysis and Algorithms: Theory and Applications, H. G. Feichtinger and T. Strohmer, Eds., Birkhäuser, Boston, 323-352.

[136] G. Kutyniok (2002). Linear independence of time-frequency shifts under a generalized Schrödinger representation, Arch. Math. (Basel) 78, 135-144.

[137] G. Kutyniok (2003). Computation of the density of weighted wavelet systems, in Wavelets: Applications in Signal and Image Processing X (San Diego, 2003), M. A. Unser, A. Aldroubi, and A. F. Laine Eds., Proc. SPIE Vol. 5207, Bellingham, WA, 393-404.

[138] G. Kutyniok (2006). Beurling density and shift-invariant weighted irregular Gabor systems, Sampl. Theory Signal Image Process. 5, 131-149.

[139] G. Kutyniok (2006). The local integrability condition for wavelet frames, J. Geom. Anal. 16, $155-166$.

[140] G. Kutyniok (2006). Affine density, frame bounds, and the admissibility condition for wavelet frames, Constr. Approx., to appear.

[141] G. Kutyniok and T. Strohmer (2005). Wilson bases for general time-frequency lattices, SIAM J. Math. Anal. 37, 685-711.

[142] H. Landau (1964). A sparse regular sequence of exponentials closed on large sets, Bull. Amer. Math. Soc. 70, 566-569.

[143] H. Landau (1967). Sampling, data transmission, and the Nyquist rate, Proc. IEEE 55, 17011706.

[144] H. Landau (1967). Necessary density conditions for sampling and interpolation of certain entire functions, Acta Math. 117, 37-52.

[145] H. Landau (1993). On the density of phase-space expansions, IEEE Trans. Inform. Th. 39, $1152-1156$.

[146] N. Lindholm (2001). Sampling in weighted $L^{p}$ spaces of entire functions in $\mathbb{C}^{n}$ and estimates of the Bergman kernel, J. Funct. Anal. 182, 390-426.

[147] P. A. Linnell (1999). Von Neumann algebras and linear independence of translates, Proc. Amer. Math. Soc. 127, 3269-3277.

[148] Y. Liu and Y. Wang (2003). The uniformity of non-uniform Gabor bases, Adv. Comput. Math. 18, 345-355.

[149] F. Low (1985). Complete sets of wave packets, in A Passion for Physics-Essays in Honor of Geoffrey Chew, C. DeTar, J. Finkelstein, and C. I. Tan, Eds., World Scientific, Singapore, 
$17-22$.

[150] Yu. I. Lyubarskii (1992). Frames in the Bargmann space of entire functions, in Entire and subharmonic functions, Amer. Math. Soc., Providence, RI, 167-180.

[151] Yu. I. Lyubarskii and K. Seip (1999). Convergence and summability of Gabor expansions at the Nyquist density, J. Fourier Anal. Appl. 5, 127-157.

[152] H. S. Malvar (1990). Lapped transforms for efficient transform/subband coding, IEEE Trans. Acoustics, Speech, Signal Proc. 38, 969-978.

[153] J. von Neumann (1932). Mathematische Grundlagen der Quantenmechanik, Springer, Berlin. English translation: "Mathematical foundations of quantum mechanics," Princeton University Press, Princeton, NJ, 1955.

[154] A. Olevskii (1997). Completeness in $L^{2}(\mathbf{R})$ of almost integer translates, C. R. Acad. Sci. Paris 324, 98-991.

[155] A. Olevskii and A. Ulanovskii (2004). Almost integer translates. Do nice generators exist?, J. Fourier Anal. Appl. 10, 93-104.

[156] T. E. Olson and R. A. Zalik (1992). Nonexistence of a Riesz basis of translates, in Approximation Theory, Lecture Notes in Pure and Applied Math., Vol. 138, Dekker, New York, 401-408.

[157] A. M. Perelomov (1971). On the completeness of a system of coherent states (English translation), Theoret. Math. Phys. 6, 156-164.

[158] J. Ramanathan and T. Steger (1995). Incompleteness of sparse coherent states, Appl. Comput. Harmon. Anal. 2, 148-153.

[159] M. Rieffel (1981). Von Neumann algebras associated with pairs of lattices in Lie groups, Math. Ann. 257, 403-418.

[160] E. Romero (2004). A complete Gabor system of zero Beurling density, Sampl. Theory Signal Image Process. 3, 299-304.

[161] A. Ron and Z. Shen (1994). Frames and stable bases for subspaces of $L^{2}\left(R^{d}\right)$ : the duality principle of Weyl-Heisenberg sets, in Proc. Cornelius Lanczos International Centenary Conference (Raleigh, NC), M. Chu, R. Plemmons, D. Brown, and D. Ellison Eds., SIAM, Philadelphia, $422-425$.

[162] A. Ron and Z. Shen (1997). Weyl-Heisenberg frames and Riesz bases in $L^{2}\left(\mathbf{R}^{d}\right)$, Duke Math. J. 89, 237-282.

[163] W. Schempp (1984). Radar ambiguity functions, the Heisenberg group, and holomorphic theta series, Proc. Amer. Math. Soc. 92, 103-110.

[164] K. Seip (1992). Density theorems for sampling and interpolation in the Bargmann-Fock space I, J. Reine Angew. Math. 429, 91-106.

[165] K. Seip (1993). Beurling type density theorems in the unit disk, Invent. Math. 113, 21-39.

[166] K. Seip (1995). On the connection between exponential bases and certain related sequences in $L^{2}(-\pi, \pi)$, J. Funct. Anal. 130, 131-160.

[167] K. Seip (2004). Interpolation and sampling in spaces of analytic functions, American Mathematical Society, Providence, RI.

[168] K. Seip and R. Wallstén (1992). Sampling and interpolation in the Bargmann-Fock space II, J. Reine Angew. Math. 429, 107-113.

[169] J. Sjöstrand (1995). Wiener type algebras of pseudodifferential operators, Séminaire sur les Équations aux Dérivées Partielles, 1994-1995, Exp. No. IV, École Polytech., Palaiseau.

[170] T. Strohmer (2006). Pseudodifferential operators and Banach algebras in mobile communications, Appl. Comput. Harmon. Anal. 20, 237-249.

[171] T. Strohmer and R. W. Heath, Jr. (2003). Grassmannian frames with applications to coding and communication, Appl. Comput. Harmon. Anal. 14, 257-275.

[172] Q. Sun (2006). Wiener's lemma for infinite matrices, Trans. Amer. Math. Soc., to appear.

[173] W. Sun and X. Zhou (2001). On the stability of Gabor frames, Adv. in Appl. Math. 26, 181-191.

[174] W. Sun and X. Zhou (2002). Irregular wavelet/Gabor frames, Appl. Comput. Harmon. Anal. 13, $63-76$.

[175] W. Sun and X. Zhou (2003). Density and stability of wavelet frames, Appl. Comput. Harmon. 
Anal. 15, 117-133.

[176] W. Sun and X. Zhou (2004). Density of irregular wavelet frames, Proc. Amer. Math. Soc. 132, $2377-2387$.

[177] M. Takesaki (1969). A generalized commutation relation for the regular representation, Bull. Soc. Math. France 97, 289-297.

[178] M. Takesaki (1979). Theory of Operator Algebras. I, Springer-Verlag, New York-Heidelberg.

[179] R. Tolimieri and R. S. Orr (1995). Poisson summation, the ambiguity function, and the theory of Weyl-Heisenberg frames, J. Fourier Anal. Appl. 1, 233-247.

[180] D. Walnut (1989). Weyl-Heisenberg wavelet expansions: Existence and stability in weighted spaces, Ph.D. Thesis, University of Maryland, College Park, MD.

[181] D. Walnut (1992). Continuity properties of the Gabor frame operator, J. Math. Anal. Appl., 165, 479-504.

[182] D. Walnut (1993). Lattice size estimates for Gabor decompositions, Monatsh. Math. 115, $245-256$.

[183] Y. Wang (2004). Sparse complete Gabor systems on a lattice, Appl. Comput. Harmon. Anal. 16, 60-67.

[184] A. Weil (1964). Sur certains groupes d'opérateurs unitaires, Acta Math. 111, 143-211.

[185] J. Wexler and S. Raz (1990). Discrete Gabor expansions, Signal Processing 21, 207-220.

[186] N. Wiener (1932). Tauberian theorems, Ann. of Math. 33, 1-100.

[187] N. Wiener (1933). The Fourier Integral and Certain of its Applications, MIT Press, Cambridge.

[188] K. G. Wilson (1987). Generalized Wannier functions, preprint.

[189] R. Young (2001). An Introduction to Nonharmonic Fourier Series, Revised First Edition, Academic Press, San Diego.

[190] J. Zak (1967). Finite translations in solid state physics, Phys. Rev. Lett. 19, 1385-1397.

[191] R. A. Zalik (1978). On approximation by shifts and a theorem of Wiener, Trans. Amer. Math. Soc. 243, 299-308.

[192] R. A. Zalik (1980). On fundamental sequences of translates, Proc. Amer. Math. Soc. 79, $255-259$.

[193] M. Zibulski and Y. Y. Zeevi (1993). Oversampling in the Gabor scheme, IEEE Trans. Signal Proc. 41, 2679-2687.

Received August 16, 2006

School of Mathematics, Georgia Institute of Technology, Atlanta, GA 30332

e-mail: heil@math.gatech.edu 